Aus der Klinik für Klinische Neurophysiologie

Prof. Dr. med. W. Paulus

der Medizinischen Fakultät der Universität Göttingen

\title{
Manipulation of the \\ Working Memory Performance in \\ Humans using Transcranial Alternating \\ Current Stimulation over the Fronto- \\ Parietal Network
}

\author{
INAUGURAL-DISSERTATION \\ zur Erlangung des Doktorgrades \\ der Medizinischen Fakultät der \\ Georg-August-Universität zu Göttingen
}

vorgelegt von

Stefanie Corinna Pabel

aus

Tübingen

Göttingen 2018 
Dekan:

\section{Betreuungsausschuss}

Betreuer/in

Ko-Betreuer/in:

\section{Prüfungskommission}

Referent/in

Ko-Referent/in:

Drittreferent/in:

Datum der mündlichen Prüfung: 15. November 2018
Prof. Dr. A. Antal

PD Dr. P. Dechent
PD Dr. P. Dechent

Prof. Dr. T. Meyer 
Hiermit erkläre ich, die Dissertation mit dem Titel "Manipulation of the Working Memory Performance in Humans using Transcranial Alternating Current Stimulation over the Fronto-Parietal Network" eigenständig angefertigt und keine anderen als die von mir angegebenen Quellen und Hilfsmittel verwendet zu haben.

Göttingen, den 


\section{Table of Contents}

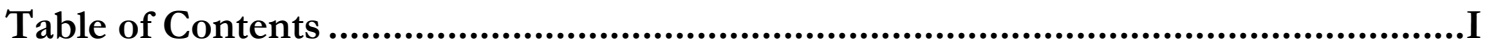

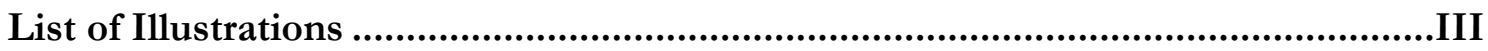

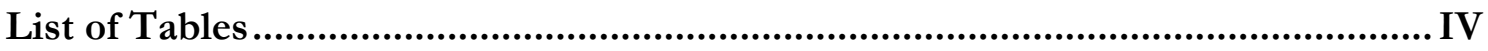

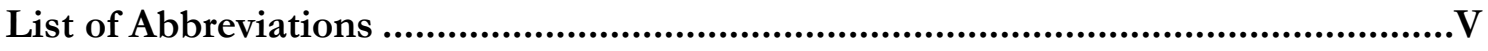

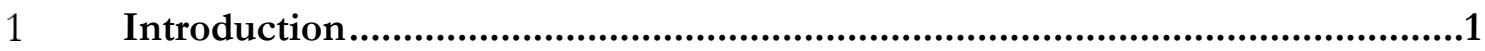

$1.1 \quad$ Memory

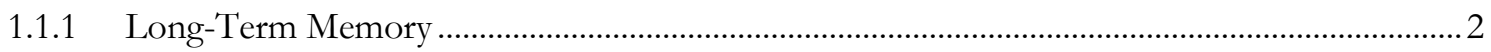

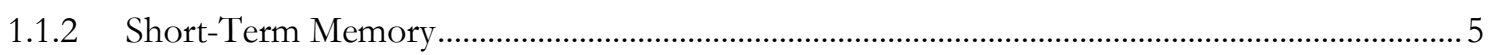

1.1.3 Differentiation from the Working Memory ......................................................................... 5

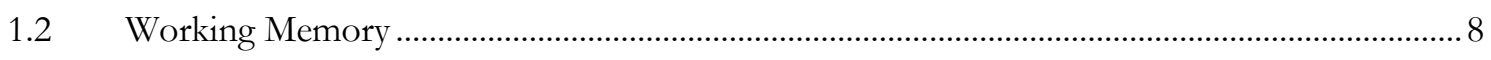

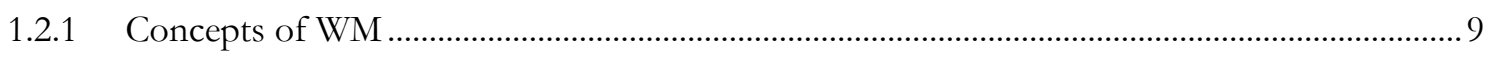

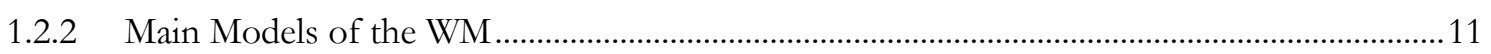

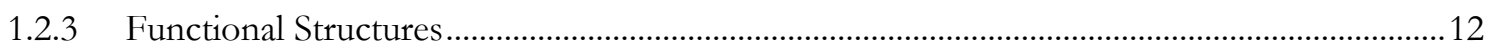

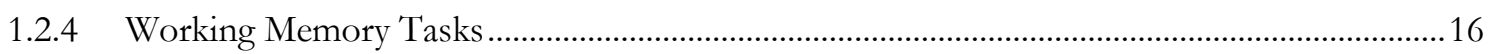

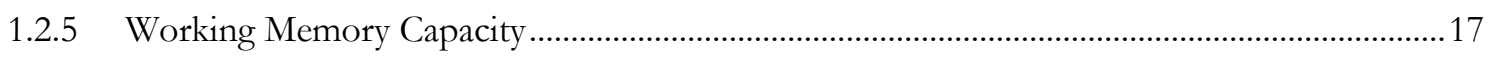

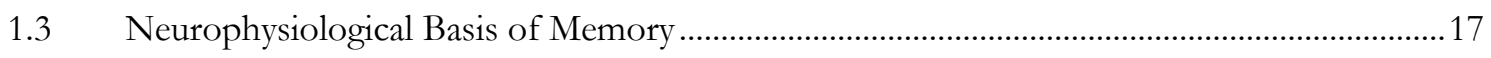

1.3.1 Functional and Anatomical Connections among Cortexes .......................................................2

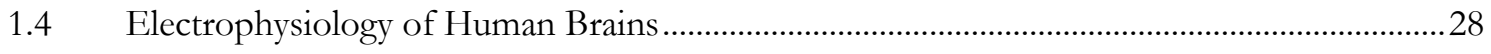

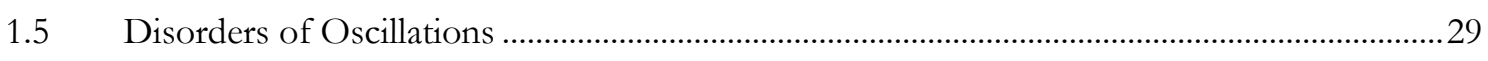

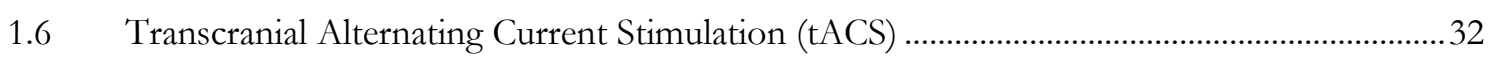

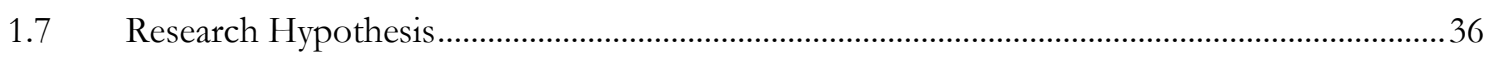

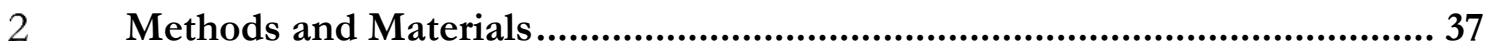

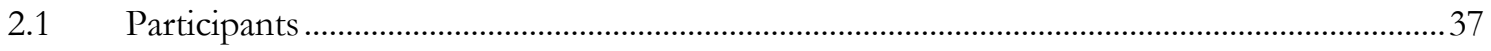

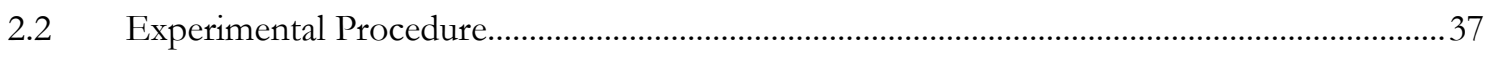

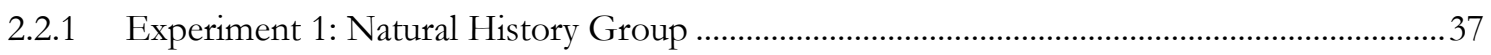

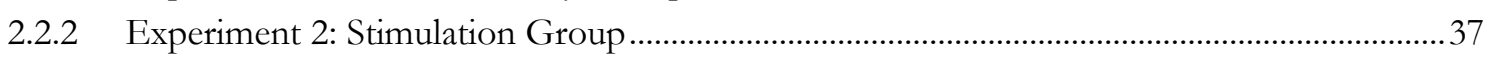

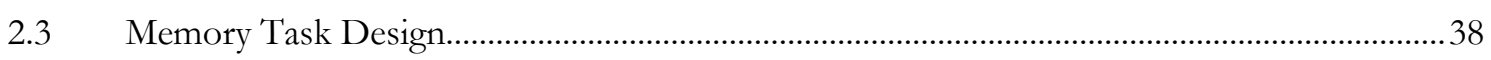

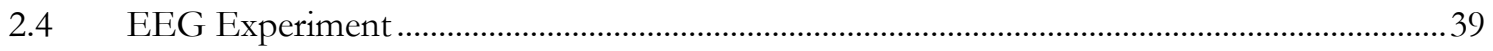

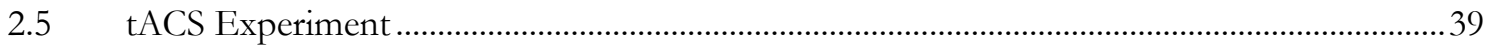

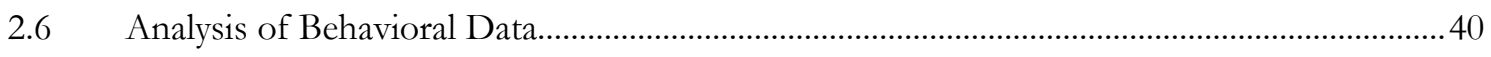

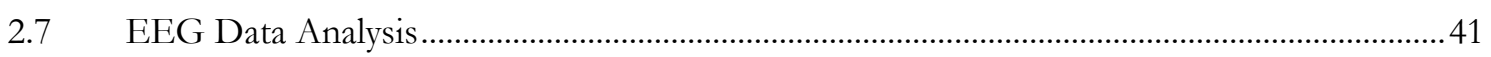

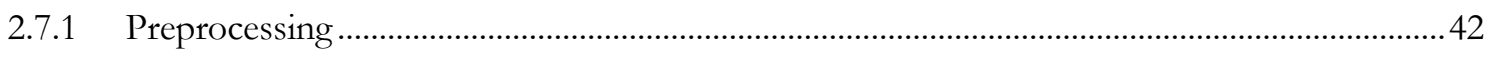

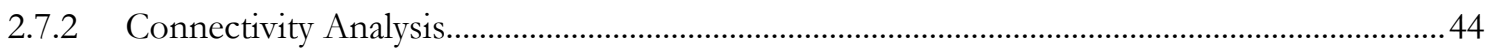

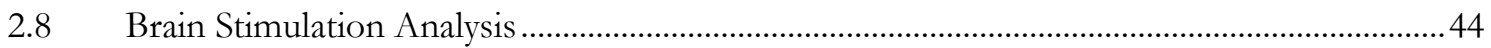

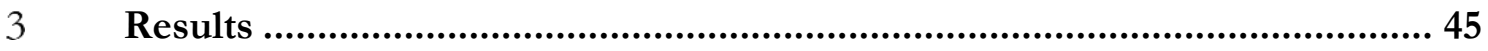

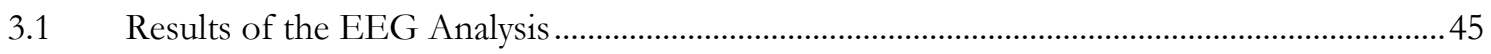




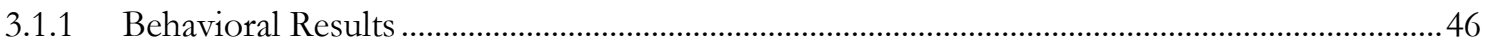

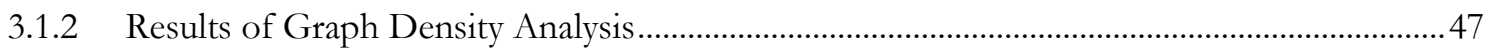

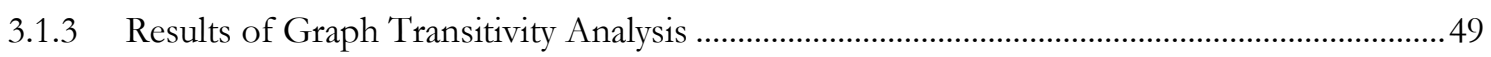

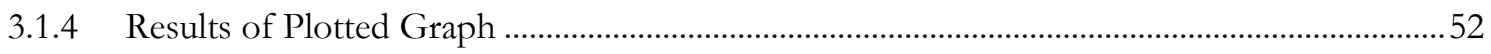

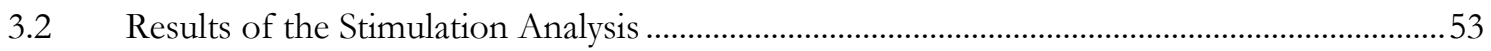

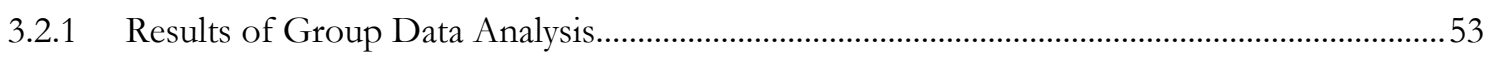

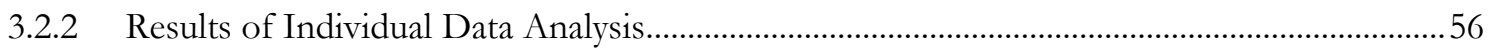

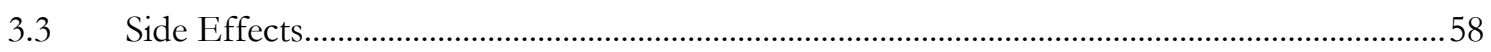

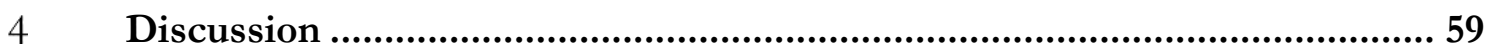

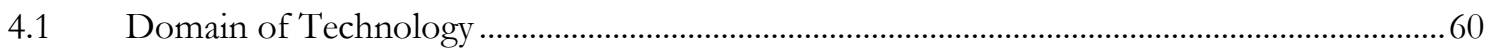

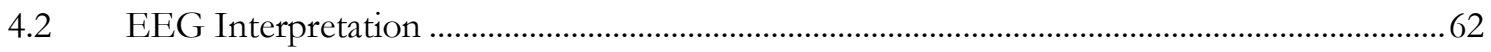

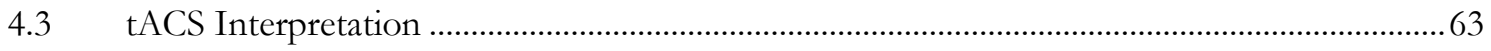

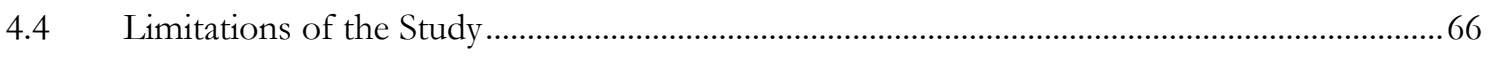

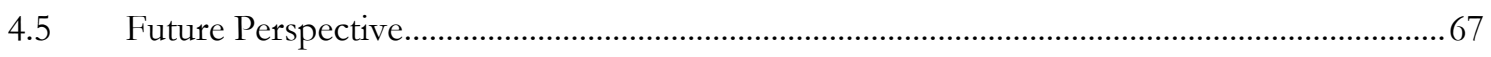

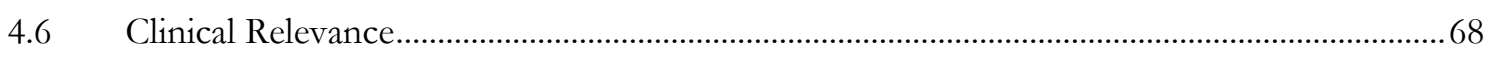

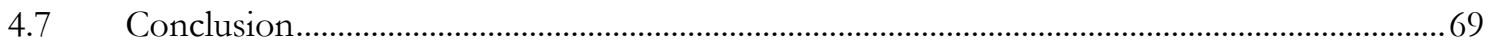

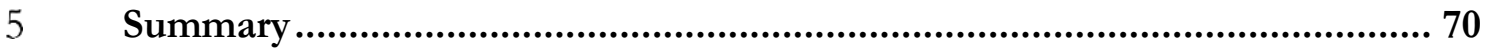

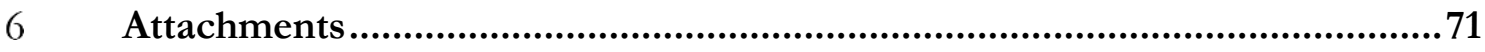

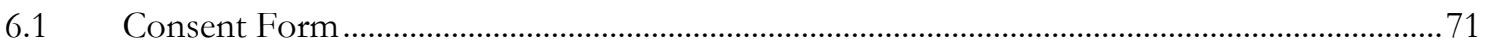

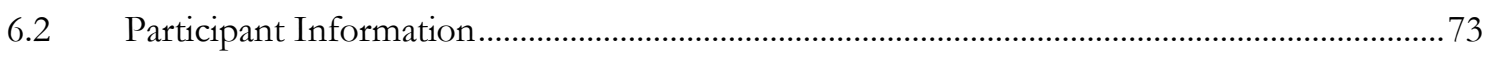

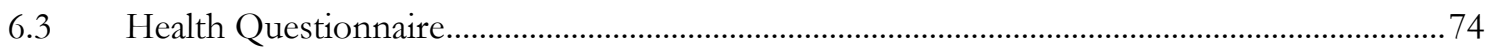

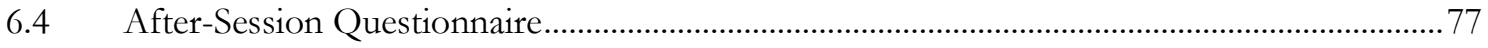

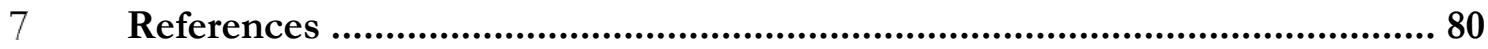

Acknowledgements ..................................................................................... 92

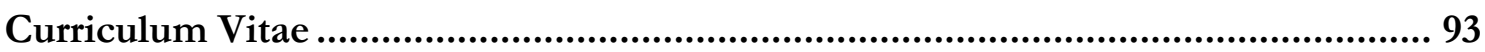

\section{Remarks}

These results have been partially published in "Alekseichuk, Pabel, Antal, Paulus (2017). Intrahemispheric theta rhythm desynchronization impairs working memory. Restorative Neurology and Neuroscience" (Alekseichuk et al. 2017). 


\section{List of Illustrations}

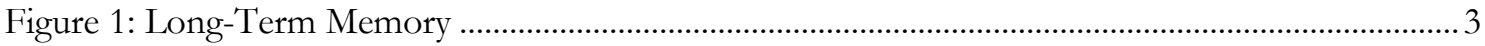

Figure 2: The Four-Component WM Model from Baddeley (2012) .................................................... 10

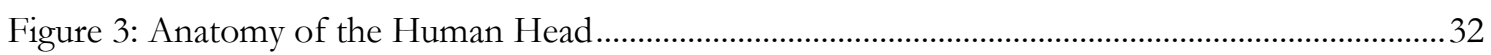

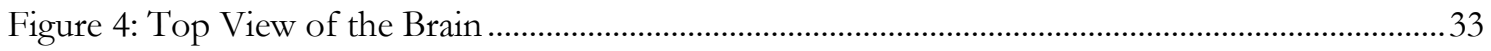

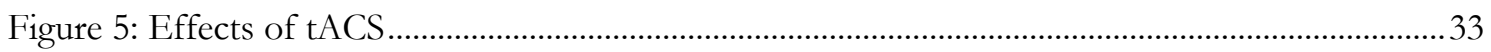

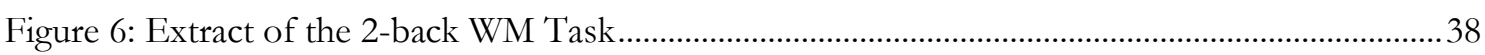

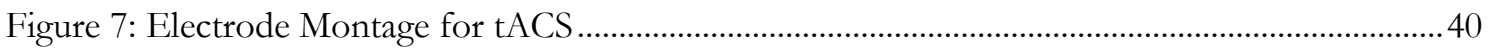

Figure 8: Interface for Trial Rejection based on abnormal Variation of the Data ..............................43

Figure 9: Independent Component Analysis ....................................................................................43

Figure 10: Pearson Correlation Coefficient for the Hit Rate, False Alarm Rate and mean Reaction

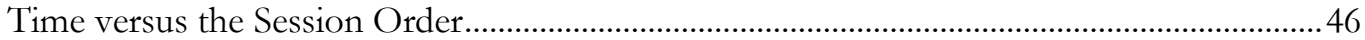

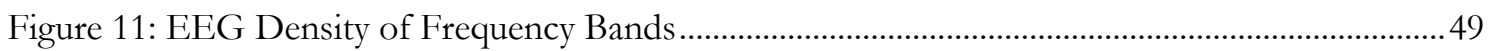

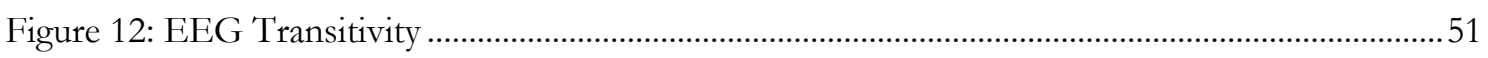

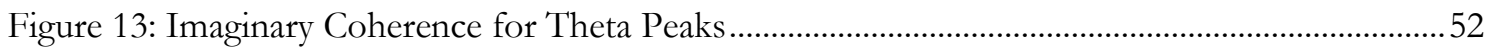

Figure 14: False Alarm Rate as a Function of tACS .....................................................................54

Figure 15: Condition specific Hit Rates in WM Task.............................................................................54

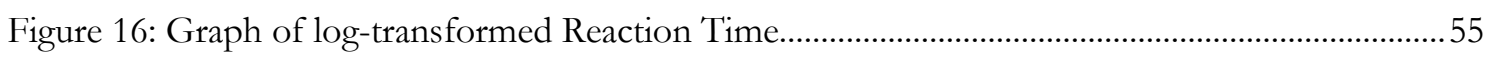

Figure 17: Graph of Working Memory Performance.....................................................................56

Figure 18: Linear Correlation between Performances for the Control Condition and

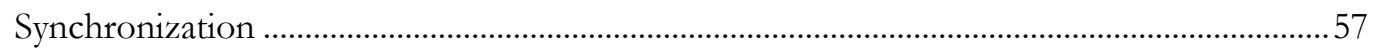

Figure 19: Linear Correlation between Performance for the Control Condition and

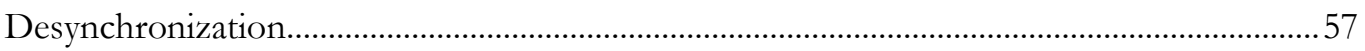




\section{List of Tables}

Table 1: Explanation of the Behavioral Parameters ………….................................................................. 40

Table 2: Behavioral Results of the Natural History Group ................................................................... 46

Table 3:. Definition of Frequency Bands and upper Confidence Interval of Density and

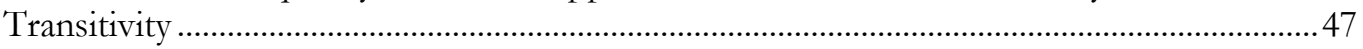

Table 4: Density, Event 1, "iCoh-" Threshold 0.1 (mean \pm SEM) ............................................................4

Table 5: Density, Event 2, "iCoh-" Threshold 0.1 (mean \pm SEM) .........................................................48

Table 6:. Density, Event 1,"iCoh-" Threshold 0.2 (mean \pm SEM) ............................................................ 48

Table 7: Density, Event 2, "iCoh-" Threshold 0.2 (mean \pm SEM) .......................................................... 48

Table 8: Transitivity, Event 1 and 2, "iCoh-" Threshold 0.1 (mean \pm SEM) .........................................50

Table 9:. Scores on Parameters for the Stimulation Group (Conditions A: Desynchronization, B:

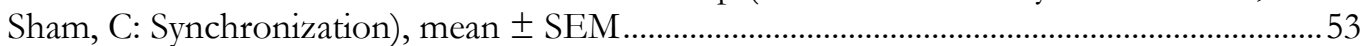




\section{List of Abbreviations}

ADHD

AMPA

BA

BOLD

CE

CI

dIPFC

DSB

DSF

ECoG

EEG

EPSP

ERP

fMRI

GABA

ICA

IPSP

LFP

LTD

LTM

LTP

NHG

NMDA

PAC

PFC

PPC

SEM

STM

tACS

TBS

TES

TMS

WM

y.o.e.
Attention Deficit and Hyperactivity Disorder

Alpha-amino-3-hydroxy-5-Methyl-4-isoxazolepropionic Acid

Brodmann Area

Blood-Oxygen-Level-Dependent

Central Executive

Confidence Interval

dorsolateral Prefrontal Cortex

Digit Span Backward

Digit Span Forward

Electrocorticography

Electroencephalography

Excitatory Postsynaptic Potential

Event Related Potential

functional Magnetic Resonance Imaging

Gamma Aminobutyric Acid

Independent Component Analysis

Inhibitory Postsynaptic Potentials

Local Field Potential

Long-Term Depression

Long-Term Memory

Long-Term Potentiation

Natural History Group

N-Methyl-D-Aspartic Acid

Phase-Amplitude Coupling

Prefrontal Cortex

Posterior Parietal Cortex

Standard Error of Mean

Short-Term Memory

transcranial Alternating Current Stimulation

Transcranial Brain Stimulation

Transcranial Electric Stimulation

Transcranial Magnetic Stimulation

Working Memory

years of education 


\section{Introduction}

In an everyday life, the human brain needs to adapt and adjust to constant changes and therefore requires the ability to maintain information and utilize it (Chaudhuri and Fiete 2016). An immense network of neurons and supporting glial cells constructs the human brain. The cerebrum is a brain section with billions of nerve cells that generates our consciousness and cognitive abilities, such as emotions, perception, motivation and memory. Most of the neuronal connections lie in a small range between neighboring brain networks, whereas long-range systems constitute an important exception, e.g. for networks with cognitive demands. In fact, the brain networks are integrating the cooperation of brain regions on different spatial scales (Siegel et al. 2012). The different neurocognitive networks that are devoted to specific cognitive functions, vary not only in structural but also in functional components (Menon 2011). Thoughts result from different cognitive systems comprising memory, attention, cognitive control, language and perception or imagery (Fröhlich et al. 2015). These short- and long-range brain systems interact through neuronal oscillations, which operate in a frequency-specific way. If parts of these networks are impaired, brain diseases can be the consequence, which highlights the precise and delicate construction.

\subsection{Memory}

How do we store memories in our mind? Memory labels the ability of the nervous system of humans and other living beings to encode, store and retrieve information and reconstitute previous experiences. Depending on the information content, memory capacity and its duration of remembrance, one differentiates between various memory storage facilities. Starting in 1890, memory models proposed a distinction between the transiently operating systems including the sensory memory, short-term memory (STM) and working memory (WM) and on the downside, the long-term memory (LTM) that represents continuous brain activity between strengthened or new synaptic contacts over a long period of time (Atkinson and Shiffrin 1968; Baddeley 2001; Baddeley 2012). The sensory memory stores images and noises for the duration of hundreds of milliseconds and has been suggested to be strongly connected to the STM (Pasternak and Greenlee 2005). Therefore, it portrays the interface between the cognitive functions of memory and perception. STM forms the storage of information for a short period of time lasting for seconds and holds the capacity for up to four visual or nine phonological information units (Miller 1956; Burle and Bonnet 2000; Cowan 2001; Kaminski et al. 2011; Ma et al. 2014). Conscious processing of sensory memory accounts for the WM, which could be compared to a 
temporary direct access storage. In comparison, information in the LTM is stored for hours up to an indefinite period (Chaudhuri and Fiete 2016). The levels of memory interact, as the WM receives information from both the LTM and the sensory memory, and simultaneously stores and manipulates their contents (Fell and Axmacher 2011). Similarly, STM contents are obtained by repetitions and the creation of short-cuts to the LTM.

Memory is a substantial part of cognition with the purpose of facilitating adaptability and personal development (Dresler et al. 2017). Past experiences and present perceptions are stored as knowledge and affect present actions. The term memory refers to the permanence of brain states over a time span, which is illustrated by transforming activity and the interaction of neuronal systems (Chaudhuri and Fiete 2016). A mental state strengthens neural activity (neural plasticity) by creating neuronal connections, which endure for either a short time span (related to the WM), or for an extended time (in the LTM), beyond the sensory perception of, e.g. the visual presentation of an object.

\subsubsection{Long-Term Memory}

According to a definition provided by Fell and Axmacher, the human LTM is a rarely limited and permanent storage system for information (Fell and Axmacher 2011). Functionally, the LTM can be classified in two types of storage facilities. It comprises the implicit (non-declarative) memory, which is subconscious, and the explicit (declarative) memory, that operates under conscious awareness, as illustrated in Figure 1 (Baddeley 2001; Schacter and Wagner 2013). The declarative memory includes the deliberate process of encoding (converting data into memory), storage and retrieval of episodic and semantic memory content. Anatomically, the temporal lobe system is active during encoding. Retrieval of explicit memory content also requires the temporal cortex and engages parietal and frontal brain regions additionally. Whereas the semantic memory stores factual knowledge, the episodic memory comprises the collection of individual and personal experiences. The non-declarative memory stores cognitive skills, actions and movements. It is not flexible and requires the original conditions of memory processes for retrieval of information. Furthermore, procedural skills, e.g. motoric learning processes and perceptual skills commonly proceed without our conscious awareness. Associative learning, non-associative learning and priming also refer to the implicit memory content. Priming defines the cognition of a stimulus that is affected by a previous presented stimulus and results in faster processing of the stimulus. Classical conditioning and operant conditioning belong to associa- 
tive learning. During classical conditioning, two stimuli are associated with each other by constantly appearing together. Operant learning implies that specific behavior is the consequence of a reinforced event, which is also entitled as the law of effect. Non-associative learning comprises the decreased response (habituation) or an intensified response (sensitization) to a stimulus. Both main types of LTM, the implicit and explicit memory, affect our thoughts and demeanor.

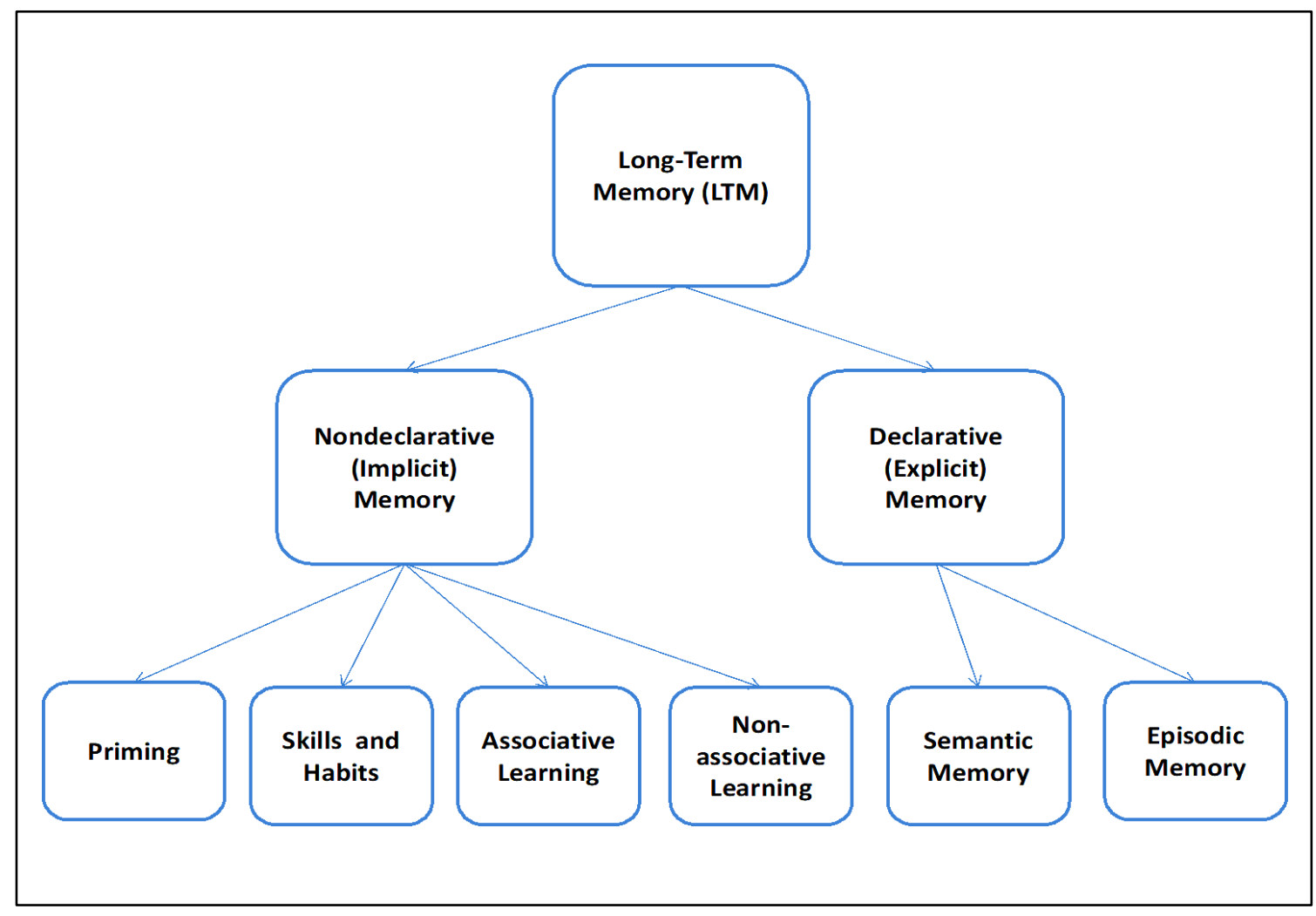

Figure 1: Long-Term Memory. The illustration is based on "Principles of Neural Science" (Schacter and Wagner, 2013, p.1447, modified).

The memory contents are not stored at a specific area in the brain but are hidden in the overall structure of nerve cells and their connections. Whereas the declarative memory is created consciously and stored in the medial temporal lobe, the non-declarative memory proceeds subconsciously and is stored in neocortical and subcortical structures. Procedural memory has been associated to the basal ganglia and the cerebellum, areas that form crucial parts in the neuromuscular control of the musculoskeletal system. The neocortical and subcortical structures are believed to interact, i.e. the amygdala, which is accountable for emotions and learning, the hippocampus that has been assumed as necessary for memory consolidation and, moreover, the rhinal cortex controls the information stream between LTM-associated areas (Simons and Spiers 2003; Fell and Axmacher 2011; Hermans et al. 2016; Loh et al. 2016). Anatomically, the declarative memory function is reported to be situated in the medial temporal lobe (Fell and 
Axmacher 2011; Schacter and Wagner 2013; Kesner and Rolls 2015; Skirrow et al. 2015). In particular, the hippocampus is an important medial temporal structure that was suggested as accountable for learning and memory processes (Cameron and Glover 2015). Information of different sensory systems is hypothesized to merge in the hippocampus, to be processed and to return back to the cortex (Bartsch and Wulff 2015). It is considered as immensely important for memory consolidation because of the information transfer from the STM to the LTM. The parahippocampal cortex and rhinal cortex are brain structures that also constitute to the medial temporal lobe for memory storage and retrieval of past experiences. Along with the hippocampus, they form the core elements for the establishment of declarative memory (Baxter 2009; Fell and Axmacher 2011). The rhinal cortex is central to perception and central processing of odors. It consists of the entorhinal and perirhinal cortex areas. Whereas the entorhinal cortex seemingly represents a multimodal center of associations, the perirhinal cortex is believed to store visual information about items (Baxter 2009).

Mnemonic mechanisms have been associated with information storage. Sensory information content is hypothesized to circulate between the sensory cortex, the rhinal and parahippocampal cortexes and to continue to the hippocampus, which gives feedback again. This circulation either leads to sorting out, or transfer to the LTM. In 1937, Papez introduced a mnemonic mechanism of how emotions and consciousness interact, later known as the Papez circuit (Papez 1937). Emotions are anatomically located in the limbic system, and their components are interconnected, which is demonstrated by the Papez circuit (Purves et al. 2008; Shah et al. 2011; Aggleton et al. 2016). According to a long-standing theory, information is temporarily stored in the hippocampus and transferred to the mammillary body by tracing the fornix. The mammillothalamic tract connects the mammillary body with the thalamus. The Papez circuit then continues from the anterior thalamic nucleus to the cingulate gyrus, located over the corpus callosum, further to the entorhinal cortex and back to its beginning, the hippocampus. Memory loss in Alzheimer's disease demonstrates that the connection between the cingulum and the hippocampus is still a relevant topic today (Aggleton et al. 2016). Furthermore, volume reductions in the hippocampus or disruptions in connections of the Papez circuit have been assumed to lead to an impaired episodic memory in patients. 


\subsubsection{Short-Term Memory}

Before information attains to the LTM storage, it is temporarily recorded and stored by the STM. This neurocognitive network processes the informational content that got into the spotlight of the attention mechanism. The memory phenomenon consciously stores information and its content is later accessible for retrieval (Baddeley 2012). The STM is in almost permanent use, as it stores visual information about imagery, musical information, as well as language content for a limited time. It has been suggested, that STM relies on either phonological or semantic coding (Baddeley 2012). Phonological coding of the STM describes a similarsounding word sequence that is being memorized. Semantic coding, in turn, refers to related words with the same meaning. A short visible image can be analyzed even though it is no longer visible and a text can be read without forgetting previous sentences. Without its functions, we could not remember experiences or events that happened immediately. Nevertheless, the storage capacity of the STM is limited, as new memory content or distraction leads to the replacement of former information. By implication, the STM must have a targeted selection to distinguish between negligible or significant content to be stored. In contrast to the unlimited LTM storage, the temporary STM also reaches its limits in terms of time. In many cases, STM content remains for only 30 seconds, unless repeated several times. Transient information can be transferred to the LTM storage and therefore may become long-term knowledge. Mentally repeating and processing has been thought to result in neuronal plasticity by an increase in the strength of synaptic contacts (Lisman 2010). Neuronal plasticity, also defined as synaptic strength, encompasses the long-term potentiation (LTP) and long-term depression (LTD). Whereas synaptic strength increases during LTP, it is also possible that synaptic contact is loosened by LTD.

\subsubsection{Differentiation from the Working Memory}

The term working memory (WM) is often used as an equivalent for the STM. However, it should be distinguished from the STM, by allocating the term STM solely to the transitory storage of items, whereas the WM also transforms the perceived information. A description of crucial functions of our WM is the following: after encoding and maintenance of information, processing of memory content takes place, while concurrently, new information is processed, and can be recalled subsequently (Duff and Hampson 2001; Cowan et al. 2005; Cowan 2010; Baddeley 2012; Roux and Uhlhaas 2014; Gignac 2015; Proskovec et al. 2016). While a variety of authors use the terms STM and WM interchangeably, this dissertation will use the definition 'WM' when referring to the manipulation of stored information and the notion STM solely in terms of temporal (short-term) storage. The WM is permanently active and enables our everyday 
life by mentally organizing exercitations, for example during planning of future events (e.g. to develop a shopping list), to comprehend spoken and written language (e.g. to read descriptions in the supermarket) and to solve calculations (e.g. to find the cheapest prize of a product) (Nissim et al. 2017).

One of the first research papers regarding the memory and its implications on personal development and individuality has been undertaken by Professor Luria (Luria 1968). Moreover, early research into the WM location has been carried out in clinical studies by functionally and structurally investigating the network in patients who suffered from lesions in the demonstrated areas (Benton 1968; Petrides 1994; Christophel et al. 2017). As a result, the anatomical location of the WM has been assigned to the key regions comprising the frontal cortex, specifically to the dorsolateral prefrontal cortex (dlPFC), and parietal cortex, particularly the posterior parietal cortex (PPC), temporal regions and subcortical structures, including the hippocampus (Müller and Knight 2006; Menon 2011; Hill et al. 2014; Zancada-Menendez et al. 2017).

Nevertheless, previous findings regarding the localization and activity of the sensory WM, visuo-spatial and verbal WM have been inconsistent, which is probably due to different conceptual formulations and experimental setups in human and primate research (Li et al. 2015). The WM is functionally subdivided into a verbal and visuo-spatial WM system (Baddeley 2012; Kawasaki et al. 2014). On the one hand, the temporal preservation and monitoring of visuospatial information was assigned to the right frontal lobe based on activation patterns, whereas the PPC has been appointed to the reorganization of stored information (Griesmayr et al. 2014; Nissim et al. 2017). On the other hand, the temporal storage of visual information has also been located in the parietal cortex (Sohn et al. 2000; Kawasaki et al. 2014). Other studies have concluded that the PPC is the central feature for the visual memory and stores information about the surrounding for a limited time (Todd and Marois 2004; Hill et al. 2014). However, the prefrontal cortex (PFC) and striatum have also been assigned to the transfer of stored information (Li et al. 2015).

Another WM distinction has been drawn for the two cerebral hemispheres. Functional magnetic resonance imaging (fMRI) studies, that combined WM tasks with imaging, have shown that especially the right hemisphere encodes information about visual and spatial features and stores information about the surrounding for a limited time (Baddeley 2000; Sauseng et al. 2005; Nissim et al. 2017). The visuo-spatial WM system engages mainly the right hemisphere. In con- 
trast, auditory (verbal) WM activity has been reported to be lateralized towards the left hemisphere (Eriksson et al. 2015). Particularly the inferior parietal lobe, lateral frontal lobe, and Broca's area, which is located in the left prefrontal lobe and crucial for language development, are involved in verbal WM processes (Hill et al. 2014; Darki and Klingberg 2015; Proskovec et al. 2016). Despite the predominant activation of the right cortex during visuo-spatial WM processing and of the left cortex during auditory (verbal) processing, recent studies suggest that the bilateral fronto-parietal network is organized in both WM tasks (Müller and Knight 2006; Nissim et al. 2017). Moreover, the occipital and temporal cortexes were placed in context with the auditory and verbal information storage (Sohn et al. 2000; Kawasaki et al. 2014). Regarding the localization of the sensory working memory, previous primate research has established that it is mainly accommodated in the PFC (Pasternak and Greenlee 2005).

Previous studies have highlighted the relevance of the prefrontal and parietal cortex activity for the WM (Olesen et al. 2004; Westerberg and Klingberg 2007). From one point, the putative role of the parietal lobe is to integrate external information. From another point, the PFC assumedly implements 'top-down control' on the (primary) sensory cortex. Nissim et al. (2017) highlighted the importance of the frontal lobe activation in complex, realistic behavior requiring the working memory (Nissim et al. 2017). The frontal lobe has been associated with cognitive processes that are largely based on the activity of dopamine-sensitive neurons, including attention, reward and motivation. According to Constantinidis and Klingberg (2016), the amount of dopamine in the frontal lobe positively correlates with WM performance (Constantinidis and Klingberg 2016). On the field of neuroscience, there is no general consensus about the separate functional roles for the prefrontal, parietal and sensory cortexes. However, there is compelling evidence that the brain regions serve as an integrated, long-range network that plays a central role in WM processing (Westerberg and Klingberg 2007; Li et al. 2015; Nissim et al. 2017). However, this view is contrary to that of Christophel (2017), who stated that brain functions are not anatomically localized but distributed between the broad networking and simultaneously activated for task processing (Christophel et al. 2017). Overall, research on the location of the WM principally agrees on the continuous activity and cooperation between the brain areas of the prefrontal and parietal cortexes and contradicts on their clear allocation of functions (Palva et al. 2010; Jaušovec et al. 2014; Christophel et al. 2017). 


\subsection{Working Memory}

Over the past century, there has been a dramatic increase in interest for the WM as a central element of cognitive functions (Constantinidis and Klingberg 2016). The feasibility of temporal retention and manipulation of visual and verbal information enables our goal-directed behavior (Sauseng et al. 2006; Darki and Klingberg 2015). The WM operates on three stages: first, encoding of a stimulus takes place, followed by the maintenance interval and finally the retrieval of memory content (Fell and Axmacher 2011). A formulated theory outlines that the WM cooperates with the LTM and sensory memory (Baddeley 2012; Kawasaki et al. 2014). The hippocampus has been suggested to facilitate the information transfer among them (Fell and Axmacher 2011). Consequently, the WM brain network performs manipulations with memory contents of new information that are maintained for several seconds and combined with nondeclarative and declarative memory contents, which are obtained from the LTM. Theoretical conceptions of WM proposed how the WM operates functionally and anatomically. A wellconfirmed theory about a multicomponent model by Baddeley and Hitch (1974) describes an interconnection between separate WM elements (Baddeley and Hitch 1974; Fell and Axmacher 2011; Baddeley 2012; Jaušovec et al. 2014). The model is based on storage systems that are controlled by an executive function, which also represents the control of attention (Cowan et al. 2005).

Where exactly is the object of interest located? With the implementation of brain imaging methods, including fMRI, spatial information about the WM has been provided. FMRI is an imaging technique that features the representation of brain activity, based on blood-oxygenlevel-dependent (BOLD) signals (Menon 2011). Signals in fMRI visualize differences in blood circulation due to metabolic processes. BOLD contrast designates the dependence of the image signal on the oxygen content in red blood cells. In this case, the BOLD effect can be used to measure neuronal activity using fMRI. Very shortly, a signal increase of the activated brain regions in MRI images is observed and explained by the fact that the neuronal activity leads to an increased oxygen consumption and initially more deoxygenated hemoglobin is detected. However, this effect is overcompensated by the hemodynamic response, whereby the cerebral blood flow is increased. Inflowing oxygenated hemoglobin finally leads to the point that the concentration of deoxyhemoglobin decreases in activated brain areas. Hereby, the observed signal, based on magnetic susceptibility, increases. By this procedure, previous investigations of Darki and Klingberg (2015) revealed a correlation between WM task performance and brain activity in the neocortical areas related to the WM. A large number of published studies describe the link between the WM and the fronto-parietal network (Bavelier et al. 2008; Palva et al. 2010; 
Takeuchi et al. 2010; Fell and Axmacher 2011; Menon 2011; Constantinidis and Klingberg 2016). The fronto-parietal network constitutes of the left and right dIPFC, the PPC, and subcortical structures (Darki and Klingberg 2015) and the functional state and anatomical features of these areas correlate with WM-related behavior in humans. It has been reported that BOLD activity in the fronto-parietal structures correlate with WM performance (Darki and Klingberg 2015). Moreover, these correlations were observed with the volume calculations of the white matter and measurements of fractional anisotropy, which characterizes the directional dependency or speed of the white matter (Takeuchi et al. 2010; Darki and Klingberg 2015). Anatomically, the dIPFC correlates with the middle frontal Brodmann area (BA) 46 and parts of BA 9 (MacDonald et al. 2000; Meiron and Lavidor 2014), and the PPC is located in BA 39/40 (Sohn et al. 2000). Furthermore, previous studies assumed that the subcortical hippocampus plays an important role in WM performance, by representing the anatomical correlative of information flow between the LTM and maintaining new information or associations between multiple features (Fell and Axmacher 2011).

In recent years, authors have begun to link brain oscillations to cognitive functions, such as to WM formation, depending on the location and their characteristic amplitude, frequency and phase (Sauseng et al. 2006; Herrmann et al. 2016; Proskovec et al. 2016). It has been assumed that theta waves represent the search process in LTM and that information is recalled by alpha oscillations, which is thought to reflect the general activation of the LTM (Sauseng et al. 2006).

\subsubsection{Concepts of WM}

What is known about the WM is largely based on accounts by Baddeley's research (Baddeley 2012). Baddeley draws a distinction between the WM and its synonym STM. Whereas WM describes the concept of temporal information storage and its manipulation, the STM only defines the storage capacity itself. A constant interaction between the WM and LTM has been described. The WM is not only required for LTM storage, but also draws information out of the LTM (Baddeley 2012). Baddeley and Hitch (1974) introduced a three-component WM model, which has remained prevailing to a certain extent and consisted of a central executive (CE), the phonological loop and the visuo-spatial sketchpad (Baddeley and Hitch 1974; Baddeley 2012; Hill et al. 2014; D 'Esposito and Postle 2015). At a later stage, Baddeley completed this model (as presented in Figure 2), by adding the episodic buffer as another component (Baddeley 2000). The fourth component serves as a link to the other components and connects the LTM with the CE. The CE has been defined as an attentional control system, which is managing other high-level cognitive functions (Menon 2011). The attentional executive 
system, which is thought to be located in the PFC (Fell and Axmacher 2011; Kawasaki et al. 2014), is crucial for cognitive processes, such as goal directed behavior during decision making and problem solving by assortment of new information (Menon 2011; Baddeley 2012). Additionally, the WM network is notably dependent on the CE, as it directs attention to transient storage and processing of information in the WM. In this way, untrained tasks are performed consciously with focused attention. On the contrary, everyday actions can also take place automatically without focused attention directed by the CE (Baddeley 2012). As displayed in the Figure 2 shown below, the CE guides the other three components, comprising the visuo-spatial sketchpad, phonological loop and episodic buffer. The multicomponent model combines visual, spatial and verbal information and facilitates the manipulation of WM contents (Hill et al. 2014).

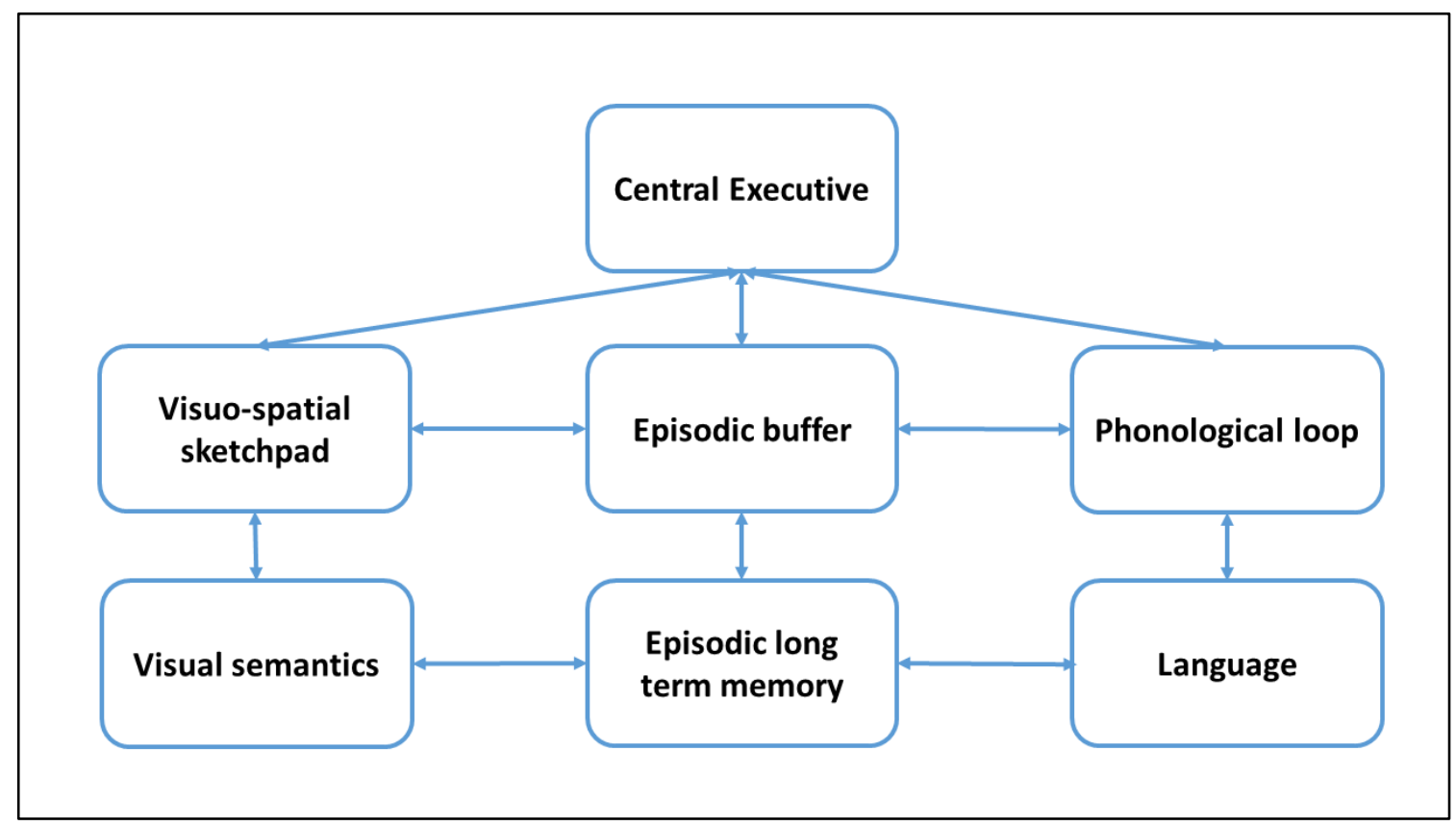

Figure 2: The Four-Component WM Model from Baddeley. The illustration is based on (Baddeley, 2012, p.16, modified).

The phonological loop is associated with the PPC and can be explained as a languagerelated temporal storage ( Schacter and Wagner 2013; Hill et al. 2014). In our minds, words are repeated perpetually 'in a memory loop' until recalled or sometimes forgotten. Similarity in words and a short word length facilitate the loop, leading to a good performance in WM tasks. Language areas, involving the Wernicke area are associated with the phonological loop (Proskovec et al. 2016). Assumedly, it has an impact on reading skills and vocabulary acquisition (Baddeley 2001). The connection with the 'phonological LTM' is a further example of the collaboration of the WM and LTM. The systems serve as an information provider, though also work isolated in separate tasks. 
The visuo-spatial sketchpad defines the WM of imagery and its performance is measured by accuracy and reaction time. It has been suggested that the capacity of the visual STM is limited to a certain number of maintained items because the accuracy decreases with an increasing amount of items (Baddeley 2012). The WM storage differs for auditory (the phonological loop) and visual items (the visuo-spatial sketchpad) (Vosskuhl et al. 2015a). It has been observed, that more visual details compared to auditory information can be memorized, thus resulting in the assumption that verbal and spatial content is stored in separate brain areas. According to Baddeley's theory, the verbal and spatial maintenance systems are essential for the short-term storage and are localized in occipito-parietal areas. Recently, the fronto-parietal and fronto-temporal networks have been associated with these systems (Baddeley 2012; Kawasaki et al. 2014).

The episodic buffer collects information from multiple memory components (e.g. from subsystems of the WM and LTM) and binds them into data blocks. Furthermore, it has been proposed that the episodic buffer links the separate storage locations for verbal and spatial information (Darling and Havelka 2010; Allen et al. 2015). Hereby, the buffer creates new imagery and new item connections after manipulation processes, and gives access to the WM and LTM (Baddeley 2001; Baddeley 2012; Hill et al. 2014). Binding of new information is required for a short period of time during WM tasks. In contrast, the binding of items in LTM processes is permanently. According to Baddeley, the WM capacity can store approximately four objects of visual conjunctions, regardless of binding conditions (e.g. a colorful figure) or separate and independent conditions (e.g. one shape). It was argued that the perpetuation of visual binding does not require the focus of attention, unless the WM is distracted by different stimuli.

\subsubsection{Main Models of the WM}

The classical view of the WM structure and its organization has been represented in the original slot model, also referred to as the item limit model (Ma et al. 2014; Cowan 2017). It describes that a fixed and small number of items is stored in the WM with high precision and this accuracy of storing the items would result in a limited WM capacity (Ma et al. 2014). According to Miller, the capacity is sufficient to accommodate seven items ( \pm two), which was demonstrated in the phonological memory tasks. However, Cowan argued that the limit for visual information is different, and equal to four items (Burle and Bonnet 2000; Cowan 2001; Ma et al. 2014; Miller and Buschman 2015). The key measure for this slot model is a fixed number of items remembered in a WM task. It describes that an item is stored in a slot and if the quantity of items exceeds the number of slots, more items are not stored. This view on the 
item limitation of the WM capacity has been challenged. Recent theoretical concepts of the WM have been designed to emphasize that the WM capacity is restricted to the quality of information that is maintained (Ma et al. 2014). In particular, it was assumed that the WM performance decelerates with an increasing amount of information but does not collapse completely (Baddeley 2012). Based on the perspective of the WM a restricted resource, new models have been generated. According to the new resource models, performance is measured by the quality and precision of the storage of memorized information content and memory retrieval (Ma et al. 2014). One of these new concepts is the equal resources model of WM. It states that the WM stores every item without a quantity limitation, but the precision of information recall declines with an increasing number of items. Conversely, the discrete representations model describes that the WM medium stores several items in separate slots (quanta) and that each item counts as either zero or one slot (Ma et al. 2014). The variable-precision model proposes that every item is stored but the quality of the information differs between each item with variance from low to high precision.

\subsubsection{Functional Structures}

How do the WM components, that are spatially separated, communicate among each other? Functional and structural studies have had a high impact on our understanding of the interaction of brain regions, including our focus, the WM. With the aid of functional neuroimaging procedures (e.g. fMRI) and electrophysiological monitoring methods, this topic was examined on a neuronal stage, by focusing on brain areas with signal changes as well as increased BOLD signal (Ma et al. 2014; Darki and Klingberg 2015). Brain regions have been demonstrated to communicate via electrical signals that are transferred by chemical synaptic contacts among neurons (Watson and Buzsáki 2013). These electrical signals occur when neurons obtain enough excitatory input to reach a certain threshold and as a result generate action potentials that induce excitatory or inhibitory postsynaptic potentials (EPSPs or IPSPs). Local changes in electroencephalography (EEG) result from assemblies of neurons that act in synchrony by generating postsynaptic potentials at the same time (Fell and Axmacher 2011). If populations of neurons create periodic electrical fluctuations (oscillations), wide spread anatomical brain regions are thought to be temporally organized and communicating with each other. This can result in topdown control, which occurs when global brain activity affects local regions of the cortex (Watson and Buzsáki 2013). The persistent activity of neurons is hypothesized to allow maintenance of information. 
The temporal organization of cognitive networks is provided by long-lasting neuronal activity of local and global brain regions and their constant communication (Watson and Buzsáki 2013). A neurophysiological model was established, in which the interaction and transmission of information among brain regions takes place via synchronous rhythmic alternations (oscillations) in the excitability of many neurons (Watson and Buzsáki 2013; Cohen 2014). Periodic oscillations are thought to reflect brain activity and are created by an electrical potential through groups of neurons firing together as cell assemblies. In this way, information is hypothesized to be transferred if groups of neurons are organized synchronously (Buzsáki 2010). Inversely, it has been argued that oscillations, generated by neuronal assemblies, have an influence on other neuronal populations throughout the cortex, by coordinating their firing rate accordingly (Uhlhaas and Singer 2010; Watson and Buzsáki 2013). Oscillations create an electrical field and the fluctuating current flow is physically measurable with electrophysiological recordings. Groups of brain oscillations that act as functional units have been divided into frequency bands. Oscillatory neuronal firing in the 4-8 Hz frequency range is referred to as theta waves and oscillations in the $40-80 \mathrm{~Hz}$ (or 30-100 Hz) frequency range are characterized as a gamma band (Burle and Bonnet 2000; Lisman 2010; Lundqvist et al. 2011; Vosskuhl et al. 2015a). There is converging evidence that frontal theta activity is the carrier frequency for coherent processes and observable during focused attention and learning (Sauseng et al. 2006; Senior et al. 2008; Cohen 2014). Particularly, increased theta activity has been allocated to WM processes, e.g. information encoding (Kirov et al. 2009).

If oscillations code for the brain communication system, however, how is simultaneous activity of distinct brain regions provided? And how is a brain network of wide-spread or local areas formed, without the interference of areas that are negligible to the intended function? A well-confirmed theory explains that brain regions communicate with the targeted brain region in their 'language', entitled resonance, which is defined by neuronal responsiveness to a specific frequency and phase (Hutcheon and Yarom 2000; Izhikevich et al. 2003; Cohen 2014; Curti and O'Brien 2016). According to this theory, neurons of a network fire in the same frequency and only respond to other neurons that are in resonance to them. Cohen (2014) compared the brain networks to a radio receiver, that tunes to the preferred frequency to receive relevant information (Cohen 2014). In addition to frequencies, phase coupling between brain regions has been linked to neuronal communication across time and space as a mechanism of information transfer between brain regions (Maris et al. 2016). Human studies examined EEG data and found either phase synchronization of oscillations between two brain regions, or an augmentation of intra-regional oscillation power, which presumably originated from synchronized 
postsynaptic potentials of groups of neurons (Fell and Axmacher 2011). The brain networks are thought to be in phase, if simultaneously activated neurons generate depolarizations, that hereby create synchronous oscillations (Battleday et al. 2014). Consistent with this argument, neuronal communication has been considered as highly impactful if the output of the sending neuronal population encounters the achieving neurons at their excitable phase (Maris et al. 2016), which in turn would result in neuronal firing in the targeted frequency. Coupling between networks is hypothesized to have a potentiation-like effect (termed LTP), which is assumed to create memory. On the other hand, oscillations are decoupled if they are firing out-of-phase (Thut et al. 2012). Decoupling of oscillations is assumed to reflect neurons that do not communicate with each other in a depression-like effect (as LTD) and result in loss of memory. Phase synchronization has also been correlated to neural plasticity (Fell and Axmacher 2011). On the one hand, Fell and Axmacher (2011) described that LTP takes place during the peak of an oscillation (Fell and Axmacher 2011). On the other hand, LTD is likely to occur during the oscillatory trough. Between two synchronized regions, information is presumed to be transmitted during high receptiveness to synaptic potentiation, namely the excitable theta phase, and information transfer would result in LTP. In summary, coordinated oscillating neurons form new connections by strengthening or weakening synaptic contact, which defines synaptic plasticity (Watson and Buzsáki 2013).

Regarding the resonance in one frequency, another behavior of oscillations could also be observed with multiple frequencies affecting each other. The relationship between two different frequency ranges has been described as cross-frequency coupling (Cohen 2011; Fell and Axmacher 2011; Thut et al. 2011). The amplitude of high frequency oscillations in local networks (e.g. gamma oscillations) can be modulated by the phase of lower frequency waves, e.g. wide spread theta waves (Herrmann et al. 2013). According to Lisman, this is the case for the WM (Lisman 2010). Synchronization through coupling of theta and gamma waves is thought to be the mechanism for the temporal storage of multiple memories in one brain network, constituting the WM. To this effect, it has been reported that low-frequency phase oscillations mainly affect oscillations of high-frequency amplitudes and thereby the latter tend to be synchronized to the former (Maris et al. 2016). As indicated, previous studies identified phaseamplitude-coupling (PAC) between theta and gamma brain waves (Canolty et al. 2009; Uhlhaas and Singer 2010). PAC belongs to a cross-frequency coupling measure, which is argued to feature an encoding scheme of communicating neuronal networks (Uhlhaas and Singer 2010). It is defined as a coupler between the phase of a slow oscillator, e.g. the theta waves, and the amplitude (equaling the square root of power) of a faster oscillator, e.g. gamma waves. The faster 
oscillations are phase-modulated by slow oscillations and therefore phase-coupled, or synchronized (Buzsáki et al. 2012).

Neural communication is seemingly affected by the interaction between frequency bands. Brain oscillations in the theta and gamma band have been recorded simultaneously during WM performance. On the basis of intracranial and EEG recordings, a model has been suggested for the WM, according to which items are maintained in WM in an oscillatory organized manner: Pursuant to the theta-gamma coding theory of STM and WM, five to nine gamma cycles are integrated in one theta cycle, which has been associated with the memory stage of maintenance (Lisman 2010; Fell and Axmacher 2011; Kaminski et al. 2011; Vosskuhl et al. 2015a). It has been assumed that each fast cycle reflects groups of neurons firing simultaneously and that this embodies one memory item (e.g. one number or letter) (Burle and Bonnet 2000). Approximately six gamma waves, representing six items, are carried and therefore organized by one slow modulating theta wave. Metaphorically, the theta wave could be compared to a shelf, which stores six books in a row, representing the gamma waves. Theta-gamma coupling first was hypothesized to be functionally specific for the WM brain state anatomically located in the fronto-parietal cortex areas (Griesmayr et al. 2010; Kaminski et al. 2011; Roux and Uhlhaas 2014). This interpretation contrasts with that from Watson and Buzsáki (2013), who argue that the frontoparietal neocortical areas also communicate with the hippocampus by cross-frequency coupling (Watson and Buzsáki 2013). The hippocampus is hypothesized to receive new information from the neocortical areas through theta-gamma coupling. In particular, hippocampal theta waves would modulate neocortical gamma waves.

Moreover, gamma oscillations have been suggested to facilitate information transfer between brain regions during bottom-up or top-down control (Fell and Axmacher 2011). During bottom-up control, subordinate brain regions, responsible for sensory processing, transfer information to higher cognitive brain regions that process the incoming sensory information. According to Uhlhaas and Singer (2010), evoked oscillations occur with a time-lag after the onset of an external stimulus and were related to sensory driven oscillatory activity and bottom-up transmission (Uhlhaas and Singer 2010). On the contrary, top-down control takes place in regions of higher cognitive processes with transfer of information to subordinate brain regions. As an example of top-down regulation, the PFC has been suggested to control the widespread parietal and temporal areas by theta phase synchronization. This mechanism was also proposed as crucial for memory formation in the LTM (Fell and Axmacher 2011). In other words, the WM and LTM seem to function by the interaction of intercortical and subcortical regions through phase synchronization (Fell and Axmacher 2011). Especially during the preservation 
of information, the fronto-parietal network assumedly communicates by phase synchronization. Indeed, a number of studies have postulated a convergence between the memory function and theta phase synchronization (Fell and Axmacher 2011;Sweeney-Reed et al. 2015; Herrmann et al. 2016). The theta phase of global areas has been proposed to organize the oscillatory activity in local areas, mainly gamma, by synchronization. This is referred to as top-down control.

Structurally, phase-coupling in the theta frequency range has been demonstrated between the PFC and the temporal lobe, i.e. hippocampal areas during all three stages of the WM process (encoding, maintenance and retrieval). According to Sauseng (2006), the PFC resembles the CE system which plies top-down control on parietal areas (Sauseng et al. 2006; Kawasaki et al. 2014). Particularly, executive control functions have been associated with synchronized theta oscillations of both the parietal and prefrontal areas (Fell and Axmacher 2011). As described above, the theta frequency is expected to be accountable for the communication between widespread cortexes, whereas the gamma frequency operates on a local level. Oscillations in the gamma frequency band have been associated with the encoding of stimuli, whereas communication between the prefrontal and parietal cortexes was shown during the maintenance of memory content (Fell and Axmacher 2011). In alignment with Helfrich's suggestion, we conclude that synchronized neuronal activity is substantial for cognition (Helfrich et al. 2014b). Synchronization facilitates the storage of new information or forgetting of former memory contents and therefore the brain is adaptable to new events. A new formation of memory is also facilitated by the remembrance of previous memory, which is stored over a longer time-span in the hippocampus and related brain areas.

\subsubsection{Working Memory Tasks}

The WM operates on three levels of encoding, maintenance and retrieval (Pasternak and Greenlee 2005; Proskovec et al. 2016). First, encoding of a stimulus defines the transformation of visual or verbal information into the WM network. Second, the WM must maintain the data and protect it from the interference to finally be able to retrieve the information upon request. In order to explore the individual WM capacity and effects of WM trainings, multiple WM tasks have been developed. In neurocognitive studies, participants are engaged in a core task requiring the WM. Effects of training can be evaluated by measuring and comparing the accuracy rates in these WM tasks before and after training. Several tasks have been developed that are associated with the activation of the fronto-parietal network, including the Sternberg-type, span-boardtype and match-non-match-type WM tasks and the N-back task (Kim et al. 2003; Meiron et al. 
2013; Buschkuehl et al. 2014; Darki and Klingberg 2015; Constantinidis and Klingberg 2016; Proskovec et al. 2016).

The N-back task is a popular delayed response task to investigate aspects of the WM. The presented target must be maintained in memory and compared to the target $\mathrm{N}$-steps back, while the previous stimulus also has to be remembered and compared to the next target. $\mathrm{N}$ stands for a natural number. In a visual N-back task, the position or color of a geometrical figure has to be compared to the stimulus presented N-steps back. The stimulus is presented on a screen and has to be remembered. At the same time, the participant is asked to compare it to a stimulus presented $\mathrm{N}$-steps back. If the presented stimulus matches the stimulus $\mathrm{N}$-back, the participant is asked to press a green button during the response period. Contrarily, the red button should be pressed if the stimuli did not match in position or color. To engage the verbal WM network in an auditory WM task, letters or words are verbally presented and the participant is asked to select the green button, if the present letter matches the letter N-back. In this work, the visual N-back WM task was selected because it specifically demands the three phases of encoding, maintenance and retrieval. The participants were trained in a 2-back-task, in which rapid sequences of stimuli (colored circles) are presented on a screen. During the stimulus presentation, the WM network encodes the stimulus with aspects of the geometric figures (visuo-spatial information about color, shape and location). In a delay period, the stimulus has to be retained in the WM and in the response period, retrieval of stimulus information takes place. Furthermore, the 2-back task specifically targets all aspects of WM: encoding, maintenance, resistance to the interference from the newer information, and retrieval. Instead of merely activating the STM, this delayed period task specifically engages the WM by requiring processing of information. Whereas the subject is asked to compare the presented stimulus of one color (purple) to the stimulus in the same color 2-back, the subject is also required to memorize the present stimulus and to compare it to the stimulus two steps later. In between the 2 steps, a cue appears in a different color, which also has to be compared to a stimulus 2-back.

\subsubsection{Working Memory Capacity}

A further distinction between the STM and WM is the number of items that can be stored. Prior to the work of Miller (1956), the STM capacity has been suggested to be limited to five to nine objects, whereas WM capacity is restricted to three to five items according to Cowan (2005) (Miller 1956; Burle and Bonnet 2000; Cowan 2001; Cowan et al. 2005; Cowan 2010; Ma et al. 2014; Cowan 2015; Cowan 2017). Miller (1956) introduced a highly effective mechanism 
to increase the number of stored items, by categorizing them into chunks. These chunks could contain digits, words or letters. For example, a list of single digits (e.g. "2,5,4,8,1,9”) could be composited to a double-digit number (“25,48,19”). In the STM tasks, adults could memorize seven \pm two items in average, reflecting little variability in memory capacity of adults. A problem definition that targets the STM is based on mental presentation and retrieval of information. For example, during a Digit Span Forward (DSF) task, a person is presented digits verbally and, subsequently, requested to recall the digits in the same order (Gignac 2015). Cowan (2005) detected a different capacity limit for the WM and claimed that adults would maintain information for only up to four \pm one objects. The Digit Span Backwards (DSB) task is a common task design that targets the WM by comprising memorization and remodeling the items (Gignac 2015). During this task, the participant is asked to memorize digits presented verbally and to recall them in an inverse order. Cowan (2005) estimated the visual capacity limit of three to four objects, by comparing the results on a visual-array comparison task. During the visual WM task, a series of items was presented, and the participants were asked to memorize the sequence. After disappearance of the stimulus, the conceptual formulation was to identify if the assortment differed or not.

Analytical results on various WM tasks are diverse. An individual WM capacity limit might rely on the storage facility itself and, furthermore, the WM efficiency depends on the focus of attention (Cowan 2010). In every assignment to the WM, we must focus our attention to the new information that is provided. Otherwise, if we get distracted by thoughts that do not concentrate on an appointed task, we might use our capacity of attention for distractions instead of storing a new amount of information that is trivial to the task. According to Cowan (2005) the ability to focus the attention and the capacity to store several objects depends on different brain areas (Cowan et al. 2005). The author labeled the capacity to focus attention on objects as the scope of attention and claimed that it would also be limited to four \pm one items. Structurally, the scope of attention was suggested to be localized in the parietal lobe and the control of attention was assigned to the frontal cortex (Cowan et al. 2005), with both interconnected in the frontoparietal network (Bavelier et al. 2008; Klingberg 2010; Palva et al. 2010; Takeuchi et al. 2010). Accordingly, strong connectivity between these areas has been argued to contribute to high WM capacity, which is of significance to this dissertation.

There are, however, more reasons why individuals differ in performance to learn or retain knowledge. Age, gender and training are displayed factors that play a decisive role in storage capacity limitation (Cowan et al. 2005; Wang et al. 2011; Hill et al. 2014; Proskovec et 
al. 2016). The temporal storage capacity is limited and variable due to individual differences in the ability to draw attention, reasoning power and education (Constantinidis and Klingberg 2016). A link has been indicated for WM capacity, childhood development and aging (Westerberg and Klingberg 2007). During childhood and adolescence, cognitive development still takes place by the generation of neurons in the later developing regions (e.g. the hippocampus, thalamus and amygdala), as the brain matures and its function, including the WM capacity, increases (Grieve et al. 2005; Cameron and Glover 2015; Constantinidis and Klingberg 2016; Proskovec et al. 2016). According to Darki and Klingberg (2015), the greater the dlPFC, superior frontal sulcus and intraparietal cortex are activated, the more extensive the WM capacity becomes in children and juveniles (Darki and Klingberg 2015). The salient activation of the PFC, especially the right dIPFC has been associated with high WM performance in adolescents (Klingberg 2010; Nissim et al. 2017). After a performance peak, the capacity commonly declines with increasing age, which was suggested to rely on neuronal degeneration (Westerberg and Klingberg 2007; Getzmann et al. 2013; Nissim et al. 2017; Oren et al. 2017). A growing body of literature has investigated the functional and cortical changes during cognitive aging and emphasized the susceptibility of the frontal cortex to structural changes (Lemaitre et al. 2012; Nissim et al. 2017). Aging is accompanied by a reduction in cortical surface area, related to the demise of synapses, and grey matter volume reduction, which mainly takes place in the PFC (Grieve et al. 2005; Lemaitre et al. 2012; Nissim et al. 2017; Zancada-Menendez et al. 2017). Nevertheless, aging does not have the same effect on every individual, as some people show maintained WM performance (Nyberg et al. 2012). Physical activity was presented as an effectual intervention to prevent cognitive decline, by facilitation of synaptic plasticity and neurogenesis, in healthy elderly and in Alzheimer patients (Intlekofer and Cotman 2013). It was investigated that senior citizens with high performance in WM, comparable with young adults, have a high activation of the PFC in both hemispheres during WM task performance (Nissim et al. 2017). The previous findings demonstrate support for the compensation hypothesis, which states that neurocognitive deterioration in elderly is counterbalanced by bilateral prefrontal activation (Reuter-lorenz et al. 1996; Fakhri et al. 2012; Proskovec et al. 2016; Nissim et al. 2017). Low performance on WM tasks consisting new memory content and its manipulation has been connected with unilateral activation of the right PFC in elderly (Nissim et al. 2017). Consequently, the structural lateralization in elderly could indicate a decline in the WM. On the neural level, a decrease in synaptic density, due to cortical surface area reduction, has also been suggested to cause a cognitive decline during aging processes. 
Gender differences have been evaluated for visual and verbal WM performance. Much of the research has paid attention to WM test achievement of males and females (Duff and Hampson 2001; Kaufman 2007). On the one hand, higher WM performance has been predicated for males on tests of spatial visualization and especially of 3D-mental rotation (Kaufman 2007), and on the other hand, for females, higher performance has been demonstrated on verbal and spatial WM tasks in shorter reaction time than male participants (Duff and Hampson 2001). Hill et al. (2014) carried out a meta-analysis to identify and evaluate the underlying neuroanatomical differences. They observed functional and structural changes during WM processes (Hill et al. 2014). Gender specific networks have been detected throughout performance of WM tasks. Stronger activation of limbic systems (e.g. the bilateral amygdala, the right hippocampus) and prefrontal areas has been demonstrated to be specific for the female gender. In contrast, males comprised more parietal areas in a dispersive WM network (viz. the superior parietal lobe, left insula, cerebellum and bilateral thalamus), which has been alleged to represent greater activation of the spatial WM. Nevertheless, Hill et al. (2014) also found gender-consistent WM network activity in parts of the frontal (BA 6,9), temporal (BA 39) and parietal lobes (BA40, BA7) and in the left cingulate gyrus (BA 32) as well as the right claustrum (Hill et al. 2014). Several investigations have revealed that training enhances the WM capacity to a certain extent (Westerberg and Klingberg 2007; Lisman 2010; Li et al. 2015; Caeyenberghs et al. 2016; Constantinidis and Klingberg 2016; Wayne et al. 2016; Dresler et al. 2017) and was suggested to be induced by plasticity (Klingberg 2010; Li et al. 2015). The method of loci is one of the oldest and well-known mnemotechnics that utilizes the spatial memory for remembrance of abstract information (Lea 1975; Anschutz et al. 1987; Qureshi et al. 2014; Fellner et al. 2016). In this method, visualizations of items are used and combined with familiar places to a route on an imaginary map.

fMRI studies revealed how brain networks were affected by mnemonic training. Different authors have measured training effects in a various of ways, comprising network connectivity, synaptic plasticity and the structure of the white matter (Klingberg 2010; Takeuchi et al. 2010; Dresler et al. 2017). Takeuchi et al (2010) investigated the structural connectivity changes of the fronto-parietal network with regard to the measurement of white fiber tracts. (Takeuchi et al. 2010). They observed increased fractional anisotropy and interpreted their results as enhanced myelination after training. In addition to these results, it has been suggested that induced plasticity changes of the frontal and parietal lobes and the basal ganglia have been interrelated with WM training. An improved performance has not only been investigated for the structural and functional cortex organization, but also for changes of synaptic connectivity (Klingberg 
2010). It was stated that synaptic plasticity precisely took place by multiplication in dopamine receptors. Another approach to understand the complex and controlled biological processes of WM training has been made by Dresler et al. (2017). Training extended widespread connections between WM networks during the state of rest, when no task was assigned. During visual task performance, the connectivity increased in local visuo-spatial brain regions, viz. the bilateral medial temporal lobe structures, ventral and dorsal networks combined, and the visual cortex. Conversely, an extensive training over six weeks reorganized the functional network connectivity over a longer period for up to four months between brain areas that were active during mental work processes (e.g. the medial PFC, right dIPFC and parahippocampal cortex).

Cowan (2010) raised the question why storage capacity is biologically restricted, and hypothesized that the processing chain is subject to neurons that fire in patterns representing up to five items in a limited time window (Cowan 2010). Greater information content might require the activation of neurons in a prolonged period that would, in turn, surpass the capacity for neural activity. Otherwise, the patterns might happen too close to each other and its interfering might mix the content in the wrong order. On the other hand, a small amount of memory content may lead to a strong association between the items and accordingly enable the most effective search process between provided information. 


\subsection{Neurophysiological Basis of Memory}

This chapter describes how memory is created on the neuronal level regarding neuronal plasticity and oscillations. Our brain must constantly adapt to new experiences. This requires the brain to memorize aspects of the environment we live in and to constantly learn from it, which is reflected by a change in human behavior. During brain development, neurons connect in a neural circuitry by forming synaptic contacts at nerve terminals. At a cellular level, learning takes place through synaptic contacts. Large neuronal ensembles encode external and internal experiences as stimuli by neural activity patterns (Citri and Malenka 2008). The brain can modify itself due to internal and external events and therefore it possesses plastic deformability. Sensory input stimulates the brain and this results in activation of neurons. Depending on the activity of neuronal structures, neuronal plasticity takes place, which is defined by the modification and plastic deformability of brain areas (Citri and Malenka 2008; Berlucchi and Buchtel 2009; Bartsch and Wulff 2015). For example, intensive exercise will increase the volume of certain involved brain regions. Hebbian learning, a cellular mechanism introduced by Donald Hebb, is supposed to take place by changing the synaptic strength for a short time (over milliseconds up to minutes), which leads to activation of the connected neuron (Fell and Axmacher 2011). New information is stored, and therefore memory is generated by strengthening or weakening preexisting synaptic contacts among neurons, which defines synaptic plasticity (Citri and Malenka 2008; Watson and Buzsáki 2013; Bannerman et al. 2014; Bosch et al. 2014; Kastellakis et al. 2015). The strength of synaptic contacts, specified by synaptic weighting, is characterized by the probability of neurotransmitter release. Neurotransmitters are biochemical substances that lead to transmission of stimuli from one cell to another and are reestablished hereinafter. Short-term synaptic weighting takes place during neural activity. Electrical impulses (action potentials) are converted into chemical information and amplified. A generated action potential in the nerve ending leads to chemical release and therefore an accumulation of calcium in the presynapse, which then discharges neurotransmitters by distributing their storage locations, the vesicles, into the synaptic gap. The neurotransmitters are either excitatory (e.g. glutamate) or inhibitory (e.g. glycine and gamma aminobutyric acid, GABA) and diffuse to the receptors of the postsynapse. This results in modification of the postsynapse by an inflow of positively or negatively charged ions when metabotropic or ligand-driven chloride channels are opened. As a result, the postsynapse is either depolarized or hyperpolarized. If the summed incoming potential reaches above the depolarization threshold, an action potential in the nerve cell is generated. After repeated activation of synapses, the stimulation has a long-lasting modification effect on the synaptic strength. According to a well-confirmed theory, the storing of memory contents is accomplished through LTP (Teyler and DiScenna 1987; Citri and Malenka 2008; Bosch et al. 2014; Cohen 
2014; Kastellakis et al. 2015; Nicoll 2017). LTP is assumed to be a learning mechanism, which operates by synaptic weighting, occurring in the pyramidal cells of the hippocampus (Bartsch and Wulff 2015). During learning, the activation of the synapse enhances the synaptic excitability. The key players in LTP are N-methyl-d-aspartic acid (NMDA)- and Alpha-amino-3-hydroxy-5-methyl-4-isoxazolepropionic acid (AMPA)- receptors, glutamate receptors located in the postsynaptic membrane that reinforce synaptic transmission over a long time. The NMDAreceptor is a protein complex which consists of four glutamatergic subunits (two GluN1 and two GluN2) (Bannerman et al. 2014). Without stimulation of the synapse, the neuron is in resting potential and the NMDA-receptors are blocked by magnesium molecules. Short series of action potentials with small glutamate efflux lead to a sodium influx through AMPA-receptors and thereby depolarization of the postsynaptic membrane. Conversely, a longer lasting series of electrical stimuli with increased glutamate efflux and stronger excitation at the postsynapse causes the NMDA-receptor to be unblocked and this especially leads to an augmented calcium influx into the nerve cell. The quantity increase of calcium, in turn, induces enzyme activation and induction of protein kinases etc. (Bartsch and Wulff 2015), which alter the postsynaptic sensitivity to glutamate or increases the presynaptic release of neurotransmitters long-lasting via the retrograde messenger substance nitrogen monoxide. A single high-frequency pulse series of depolarizations phosphorylates the AMPA-receptors for one to two hours, whereas a high amount of calcium affects the protein biosynthesis. The resulting modification of synapses endures for hours to weeks. On the contrary, LTD takes place if synaptic contact is weakened, and therefore, the theory also provides a comprehensive explanation of how memory is forgotten. Neural plasticity via LTP and LTD is not the only mechanism of memory formation. Another integral neurophysiological aspect in memory formation is the temporal pattern of hippocampal neuronal skipping. According to this hypothesis, new formation of memory is facilitated by the reactivation of previous memory that is stored in the hippocampus and related brain areas. Successful retrieval of information might not primarily rely on the total augmentation in postsynaptic potentials, but rather on the temporal precision of hippocampal neuronal firing (Voytek and Knight 2015). Overall, synaptic plasticity is a structural mechanism that modifies conjunctions between synapses and hereby underlies learning and memory (Schacter and Wagner 2013).

A critical aspect of neurophysiological research is to gain a deeper insight into the formation of brain activity and its relevance in memory. Brain activity occurs at the nerve cell level in terms of oscillations and interactions with larger brain areas. Neuronal oscillations reflect fluctuations in neuronal states of excitation and inhibition, but also mere excitatory or inhibitory 
brain networks (Cohen 2014). It is a widely-held view that oscillations are produced by ongoing synchronous activity of neuronal assemblies, which fire in rhythmic bursts (Roux and Uhlhaas 2014). The characteristic cycles of these bursts create oscillations, which in turn create changes in the electric potential surrounding the neurons, entitled as the local field potential (LFP). LFPs are compiled of postsynaptic potentials from synchronized neuron ensembles. These signals are central targets in brain research: Electrocorticography $(\mathrm{ECoG})$ is an invasive research method that uses intracranial single-unit electrodes or multi-unit electrodes to record extracellular electric potentials (Fell and Axmacher 2011; Buzsáki et al. 2012; Voytek and Knight 2015). Hereby, brain activity is measurable. Functionally, neuronal oscillations are described as a system with a constant rhythmic shift between brain states (Cohen 2014) and suggested to transmit encoded information between neuronal populations (Roux and Uhlhaas 2014). Brain oscillations are characterized by three features: the frequency, power and phase (Cohen 2014). The speed of an oscillation shapes the frequency that is calculated per unit Hertz $(\mathrm{Hz})$ and measured in cycles per second. The height of an oscillation defines the amplitude of the brain wave and the squared amplitude results in the power, its amount of energy. Inversely, the amplitude of an oscillation equals the square root of power. The phase is a circular measure in radians or angles and describes each time point within a cycle. Phase can also be transformed to the oscillation's time point or a certain position in oscillations (Thut et al. 2012). The phase of oscillations is referred to as either excitatory (the peak), inhibitory (the trough), or in between (the slope). Thut et al. emphasized the role of variances in phase, frequency and amplitude for oscillations and its modifiability by neuronal interaction (Thut et al. 2012). The phase of neuronal oscillations can provide additional insight into the temporal organization of cortical activity (Roux and Uhlhaas 2014). The up-states of oscillations reflect a time window of greater excitability, and the downstates stand for a less excitable period. As stated above, brain oscillations contain three dimensions of neuronal information (frequency, power and phase). The speed of the oscillation characterizes the frequency. Power refers to the height, or the amount of energy, in the oscillation at a point in time. The phase is a circular measure in radians or angles and reflects the position of the oscillation along the sine wave (Cohen 2011). During the excited state, the sine wave's phase is termed as the peak, $(\pi)$ and on the contrary, the trough $(-\pi)$ reflects the unexcited state of the neuronal network.

Brain wave patterns can be observed with non-invasive EEG, which records the electrical signal from electrodes mounted on the scalp (Battleday et al. 2014). According to Lopes da Silva (2010), the EEG receives summed electrical activity of neurons and glial cells that generate electrical and magnetic fields (Lopes da Silva 2010). In the following, the purpose of each brain 
wave will be explained and linked to specific functions. Through filtering of electrical signals, brain oscillations are typically divided into different frequency bands that are referred to as the delta $(1-4 \mathrm{~Hz})$, theta $(4-8 \mathrm{~Hz})$, alpha $(8-12 \mathrm{~Hz})$, beta $(15-25 \mathrm{~Hz})$, and gamma frequency band (40-80 Hz) (Battleday et al. 2014; Hanslmayr et al. 2016). According to literature, the frequency bands are varying in bandwidth. Neural communication and their behavioral results in cognition have been linked to neural oscillations in the frequency bands between delta and gamma (Voytek and Knight 2015). In particular, studies have reported that the performance on several tasks is predicted by the power and phase in oscillations (Voytek and Knight 2015). The slowest frequency, delta oscillations, has been found in the frontal and cingulate cortex and is assumed to constitute a key element for attention by inhibiting insignificant sensory stimuli and visual information (Herrmann et al. 2016). Oscillations in the delta and theta band have been associated with the communication of brain networks in a longer range and hereby enable higher cognitive processes, e.g. learning, speech comprehension, focused attention and WM processes (Griesmayr et al. 2010; Kikuchi et al. 2015; Herrmann et al. 2016). Theta band activity is especially significant during memory recall and spatial navigation. Repetitive neural activity in the theta range was anatomically localized in the PFC, sensory cortex, as well as parietal and temporal areas with mainly the hippocampus that has been suggested to play a crucial role in episodic memory encoding (Khader et al. 2010; Uhlhaas and Singer 2010; Herrmann et al. 2016; Hanslmayr et al. 2016). In addition to theta oscillatory activity, theta and alpha oscillations combined have been shown to regulate encoding of the LTM (Khader et al. 2010).

For the occipital cortex, dominant rhythmic activity in the alpha band has been described and was related to eye-opening and closing in a relaxed and awake state in animals and humans (Lopes da Silva 2010). The power increases during an eyes-closed state, whereas opening of the eyes results in decreased alpha rhythm and increased beta activity in the visual cortex. It should be mentioned, however, that eye closure has also been reported to result in widespread power increases in delta, theta and beta power and occur beyond the occipital areas. Geller et al. described this change in frequency power in a study using ECoG, recording cortical activity by directly placing electrodes on the cerebral cortex. Additionally to the occipital cortex, alpha rhythm has also been examined to be generated in the posterior temporal and parietal cortices, including the thalamus, hippocampus, reticular formation, sensory cortex and motor cortex (Uhlhaas and Singer 2010). It is assumed to reflect memory processes and facilitate attention control by suppressing irrelevant visual information input (Uhlhaas and Singer 2010; Battleday et al. 2014; Herrmann et al. 2016). The sensory inhibition facilitates sensory and memory activity by focusing on the relevant information. Furthermore, previous studies have discovered theta 
and alpha band activity modulations during top-down processes and found a connection through synchronization between widespread brain areas (Uhlhaas and Singer 2010; Battleday et al. 2014).

Whereas Uhlhaas and Singer claimed that beta oscillations occur in all cortical structures, and subcortical areas such as the subthalamic nucleus, basal ganglia and olfactory bulb, Battleday et al. highlighted the role for motor cortex functions (Uhlhaas and Singer 2010; Battleday et al. 2014). During sensory and motor tasks, as well as tasks that require cognitive ability, beta band activity increases during planning of fine motor skills. This performance is enabled by filtering out unnecessary stimuli to focus the attention on essential information, which defines sensory gating (Uhlhaas and Singer 2010; Herrmann et al. 2016). Gamma band activity has been recorded in local cortical areas of all brain structures, as well as the retina and olfactory bulb (Griesmayr et al. 2010; Uhlhaas and Singer 2010; Herrmann et al. 2016). It was correlated to conscious perception, attentional control during processing of information and WM maintenance (Griesmayr et al. 2010). Moreover, gamma oscillations have also been suggested as the facilitator of LTP by initiating synaptic changes associated with learning (Hanslmayr et al. 2016).

Interactions between two or more frequencies have been assigned to the communication of neuronal networks due to the coordination through frequency coupling (Thut et al. 2011). Communication of long-range networks is assumed to be facilitated by oscillatory activity of slow frequencies (in the theta- to alpha-range), whereas communication with higher frequencies is primarily hypothesized to take place between local networks of neurons. Occipital gamma oscillations are hypothesized to combine visual features as soon as posterior alpha-frequency oscillations have selected and integrated information designated to be important (Thut et al. 2011). Memory formation, resulting from LTP, has been demonstrated to arise mainly during specific phases of theta-band oscillations (Cohen 2014). This result may support the hypothesis claiming that episodic memory would rely on information binding through gamma oscillations and are itself regulated by theta oscillations (Hanslmayr et al. 2016). 


\subsubsection{Functional and Anatomical Connections among Cortexes}

Brain functions are particularly relevant in maintaining the physiological homeostasis, cognition and motor control and facilitated by the precise and flexible coordination of intraand interregional neuronal activity (Voytek and Knight 2015). Oscillations represent this activity and can be generated by populations of neurons that either spike according to the same oscillatory frequency or contradictory. The theory of oscillatory synchronization, or coherence, provides a useful explanation of how interregional network communication is facilitated in the brain (Voytek and Knight 2015). Coherence is defined by the correlation of power. Two oscillations are coherent if their frequencies are identical and if they are aligned in phase and amplitude. The mechanism behind this effect is explained by neuronal spiking activity that occurs in the same phase. The coupling of neurons therefore takes place via phase-synchronization and during coherence in phase, spikes are more likely to occur. Spiking activity of neuronal populations, represented by action potentials, would then enhance the LFP, which represents the summed activity. The LFP in turn is thought to regenerate oscillatory synchronization of further neuronal networks. By contrast, if neurons are decoupled, their spiking activity takes place during individual phases. Thereby, neural noise is created and it reduces the probability for the generation of action potentials. Moreover, Voytek and Knight (2015) suggested that oscillatory coupling is thought to be well optimized for successful and coordinated neuronal spiking activity (Voytek and Knight 2015). On the one hand, oscillatory neuronal activity should not be overly synchronous with severe coupling, and on the other hand, it should not be impaired by weak coupling. In either way, it is assumed that information transfer among networks would be impaired. 


\subsection{Electrophysiology of Human Brains}

One of the most well-known and used tool for assessing electrical activity of the brain is EEG (Buzsáki et al. 2012). The EEG is defined as a non-invasive neurological measurement that derives from summed cortical potential fluctuations. It captures brain wave patterns as rhythmic activity and amplifies the signal recorded passively through electrodes mounted on the scalp. A major advantage of the brain imaging technique is it's high time resolution, which measures occurring cognitive processes and alterations in real time (Cohen 2014). The EEG measures electrical signals from the brain that travelled through the dura layers, skull layers, the scalp and intermediate tissue, and also captures noise that arises from the surrounding (Buzsáki et al. 2012; Jackson and Bolger 2014).

The major source of the EEG signal originates from electric fields that are generated by synchronized activity of cortical neurons (Thut et al. 2012; Watson and Buzsáki 2013; Cohen 2014; Jackson and Bolger 2014). The EEG measures the state changes of neurons summed up at different points of the cortex and thus visualizes the activity of groups of neurons. Rhythmic activity in the EEG is registered through these voltage fluctuations. A number of authors have considered the effects on the signals of pyramidal cells in the cortex as the greatest (Buzsáki et al. 2012; Jackson and Bolger 2014; Chaudhuri and Fiete 2016). In particular, the summation of their generated postsynaptic potentials contributes mainly to the recorded electrophysiological activity (Voytek and Knight 2015). If the neurons are geometrically organized, either parallel or perpendicular and along cortical columns, they create an extracellular field voltage. Dipole moments are generated by dendrites and the soma of pyramidal cells that are separated by some distance and charged contrarily. The positive charged field serves as the source and arises from positive current, which excites the neuron at the neuronal membrane (Buzsáki et al. 2012; Jackson and Bolger 2014). On the contrary, the sink defines a negatively charged region. If multiple apical dendrites of pyramidal neurons are parallel arranged and synchronously active, the dipole moments sum together and produce a measurable signal that is detected by electrodes in the vicinity (Buzsáki et al. 2012; Jackson and Bolger 2014). In this way, the EPSPs or IPSPs of many neurons summate and create an extracellular potential near the neural dendrites (Cohen 2014; Jackson and Bolger 2014). As EEG records an electric field from outside the neurons, the neuronal action potentials are not directly recorded. Instead, action potentials and synaptic activity produce voltage in the extracellular space. This voltage, an electrical potential of multiple neurons, sums up to an extracellular signal, and can be detected on the smaller scale as LFP. The electric potentials derive from transmembrane currents. The LFP is an extracellular current flow which is measured by intracranial EEG recording electrical activity from the cerebral cortex by electrodes placed inside of the brain. Small synaptic currents produce a measurable LFP 
signal by overlap in time. The slow synaptic activity generates a substantial electrical current flow and therefore accounts for the extracellular voltage measured from the scalp (Buzsáki et al. 2012). There is converging evidence that other transmembrane currents also contribute to the signal, including voltage-dependent intrinsic events, e.g. calcium spikes, fast action potentials and spiking after hyperpolarization. Calcium spikes are large (10-50 mV), long-lasting (10-100 ms) and often triggered by NMDA receptor-mediated excitatory postsynaptic potentials (EPSP). Fast action potentials generate from fast sodium-channels in open positions, which can externally be observed as spike activity in the extracellular medium lasting for about two milliseconds. Synchronous action potentials from many neurons can contribute to the LFP. Neuronal synchrony may also be augmented by direct electric communication (gap junctions and neuron-glia interactions) that can contribute indirectly to changes in the extracellular field (Buzsáki et al. 2012). The elevation of intracellular concentration of ions may activate ligandgated channels and therefore lead to an increase of conductance of other ions, and finally create hyperpolarization of the membrane.

\subsection{Disorders of Oscillations}

Neurophysiological studies have linked brain oscillations with mental work including cognitive processes and changes in cognition were suggested as externally observable through human behavior (Uhlhaas and Singer 2010). Many neuropsychiatric and learning disorders, e.g. Autism and Depression, were associated with pathological oscillatory brain activity in large-scale networks (Menon 2011; Herrmann et al. 2013; Battleday et al. 2014; Voytek and Knight 2015). Moreover, the disruption of the fronto-parietal network and abnormal central executive functions has been linked to the complex mental disorders Attention Deficit Hyperactivity Disorder (ADHD) and Schizophrenia (Martinussen et al. 2005; Klingberg 2010; Menon 2011; Li et al. 2015). In this chapter, the relevant disorders will be presented and brought into the context of potential treatment with brain stimulation.

ADHD is a prevalent neurodevelopmental disease in childhood, that affects approximately 3\%, and often pervades through adolescence and adulthood (Menon 2011; Rodríguez et al. 2016; Groenman et al. 2017). Disruptions in the critical phases of the development of the brain were described to precede ADHD, which is predominantly characterized by unfocused, impulsive and hyperactive behavior that is age-inappropriate and lasts over a long period of time (Groenman et al. 2017). A search of the literature revealed that the severity of ADHD correlates with cognitive deficits regarding an impairment of the WM in patients (Westerberg et al. 2004; Martinussen et al. 2005). ADHD can be divided into three types of presentations: the impulsive 
and hyperactive type, the inattentive type, and a combination between both types (Rodríguez et al. 2016). In early childhood, especially young male individuals attract attention from nursery and school teachers' due to impulsive and hyperactive behavior. The inability to stay focused and pay attention leads to difficulties particularly at school and influences the social development among peers. On these grounds, pharmacological treatments with methylphenidate and atomoxetine find widespread use. Despite the positive effects on cognition and motor skills, this sympathomimetic agent also generates adverse effects that include tachycardia, increasing blood pressure, sleep disturbances and aberrant sweating. It has been suggested and also disproved that the increased amount of dopamine could also play a role in the reduction in growth by diminishing the amount of growth hormone that is distributed (Bereket et al. 2005; Groenman et al. 2017). Although the safety of stimulants regarding short-term effects and adverse reactions is well investigated, the long-term impact of methylphenidate on brain development and the cardiovascular system is not yet well established. In contrast to the pharmacological approach and treatment of ADHD, the brain activity of patients has been investigated and compared with healthy individuals. Previous research that implemented fMRI led to the conclusion that methylphenidate has a positive reinforcing or compensatory direct effect on the functional network connectivity and patterns of activation (Rodríguez et al. 2016). Surveys conducted by Herrmann (2013) have shown abnormal oscillatory brain activity in patients suffering from ADHD. With regard to the impaired memory performance in patients, a relationship has been drawn between the degree of cognitive deficits and the decrease in EEG amplitude of gamma-band activity (Herrmann et al. 2013).

A further neurodevelopmental disorder that is noticed and diagnosed in childhood, is the Autism Spectrum Disorder (ASD) (Kikuchi et al. 2015; Bonnet-Brilhault 2017). The term falls in a category of Pervasive Neurodevelopmental Disorder, which includes not only autism, but Asperger syndrome and Pervasive Neurodevelopmental Disorder not otherwise specified. Autism is defined by an early onset in the first three years in children with a characteristic set of symptoms: deviants and restricted interest in social communication and social interaction, as well as repetitive behavior, sensory-motor dysfunctions and altered executive functioning. The first symptoms can already occur in the age of 18 months and in many cases, people with autism are also diagnosed with comorbidities such as ADHD. Autism is caused by environmental factors as well as an undetermined genetic component. During brain development, the neuronal organization and neural connectivity in patients differ in comparison to their peers, which is suggested to lead to salient social behavior. Previous research findings regarding the altered brain connectivity have found reduced theta coherence $(6 \mathrm{~Hz})$ and decreased network connections in the anterior-posterior direction of the diagonal hemispheres (Kikuchi et al. 2015). 
The psychiatric disease schizophrenia has also been associated with dysfunctional brain networking (Henseler et al. 2010). Approximately 1\% of the world's population is suffering from schizophrenia, which can be defined as a complex mental disorder. Schizophrenia is identifiable by psychotic symptoms, such as hallucinations, delusions and impairments in cognitive functions, as well as a genetic component (Uhlhaas and Singer 2010; Menon 2011; Devaraju et al. 2017). Patients with schizophrenia suffer from cognitive disorders encompassing language, disorganized speech, reasoning, thinking and planning, and deficient WM performance. The psychotic symptoms, e.g. auditory hallucinations, have been shown to predict disease progression. This highlights the importance for patients.

Global and local disorders in the brain network are associated with schizophrenia and have been shown to impact cognitive abilities of patients (Menon 2011). The disease particularly influences the visuo-spatial and verbal WM in the temporal, parietal and frontal lobe in a pathogen manner (Henseler et al. 2010). As mentioned above, oscillations in the theta and alpha band (slow frequencies) were demonstrated to synchronize widespread brain areas (large-scale networks), whereas synchronization of beta and gamma-band activity (higher frequency) has been observed in regional areas, reflecting communication in a small-scale (Herrmann et al. 2016). To this effect, there is converging evidence that synchronization between beta and gamma oscillations is abnormal in patients suffering from schizophrenia and that this abnormality has not been able to be treated by pharmaceuticals (Uhlhaas and Singer 2010). Previous research has suggested that abnormalities in oscillations and synchronization are present in patients and do not result from antipsychotic medication. More precise, schizophrenia has been associated with a reduction in brain volume, as well as the grey and white matter and the ventricular volume, which probably results in cognitive decline (Uhlhaas and Singer 2010). According to Uhlhaas and Singer (2010), the grey matter volume, which is associated with the amount of connected synapses, is reduced in patients although the total amount of neuronal cells is not altered (Uhlhaas and Singer 2010). Finally, research in neuropsychiatric disorders focused on schizophrenia and identified reduced connectivity in fronto-parietal and fronto-temporal regions (Menon 2011). Furthermore, electrophysiological findings revealed an abnormal decrease in activity of high gamma amplitudes and low theta oscillations over the frontal cortex during resting state, and reported a reduction in theta coherence (Uhlhaas and Singer 2010). This is an important finding regarding to the part of our study that describes the functional connectivity changes (see below). A genetic component in the development of this disease has been circumstantiated by Uhlhaas and Singer (2010). It was claimed that the malfunction would be inheritable. In patients and their first degree-relatives, similar results on disrupted oscillations in the alpha and theta band were found. This raises the issue of whether it could be possible to reduce 
these symptoms and decelerate disease progression by synchronizing WM related cortical areas. Albeit the main brain region of cognition, the hippocampus in the medial temporal lobe, could not be reached by non-invasive transcranial stimulation.

Patients suffering from depression experience symptoms of persistent sadness and the sense of worthless as well as the feeling of helplessness (Menon 2011). They frequently experience phases in which they are abnormally anxious, sad and pessimistic about their life and its purpose. The depressive episode of patients with major depressive disorder has been shown to correlate with impaired memory (Watson and Buzsáki 2013). Moreover, deficits in the activation of the CE network, anchored in the dIPFC and PPC, have been associated with the cognitive disorder (Menon 2011).

\subsection{Transcranial Alternating Current Stimulation (tACS)}

What is the causal role behind the synchronization of oscillating brain networks? This question has been approached by demonstrating that interference in brain activity could both impair and improve functions of targeted networks and modify related behavior (Fröhlich et al. 2015). Electrical stimulation applied on the scalp has been shown to create an electric field, and this causes an interaction with neuronal oscillations and spiking activity. Transcranial alternating

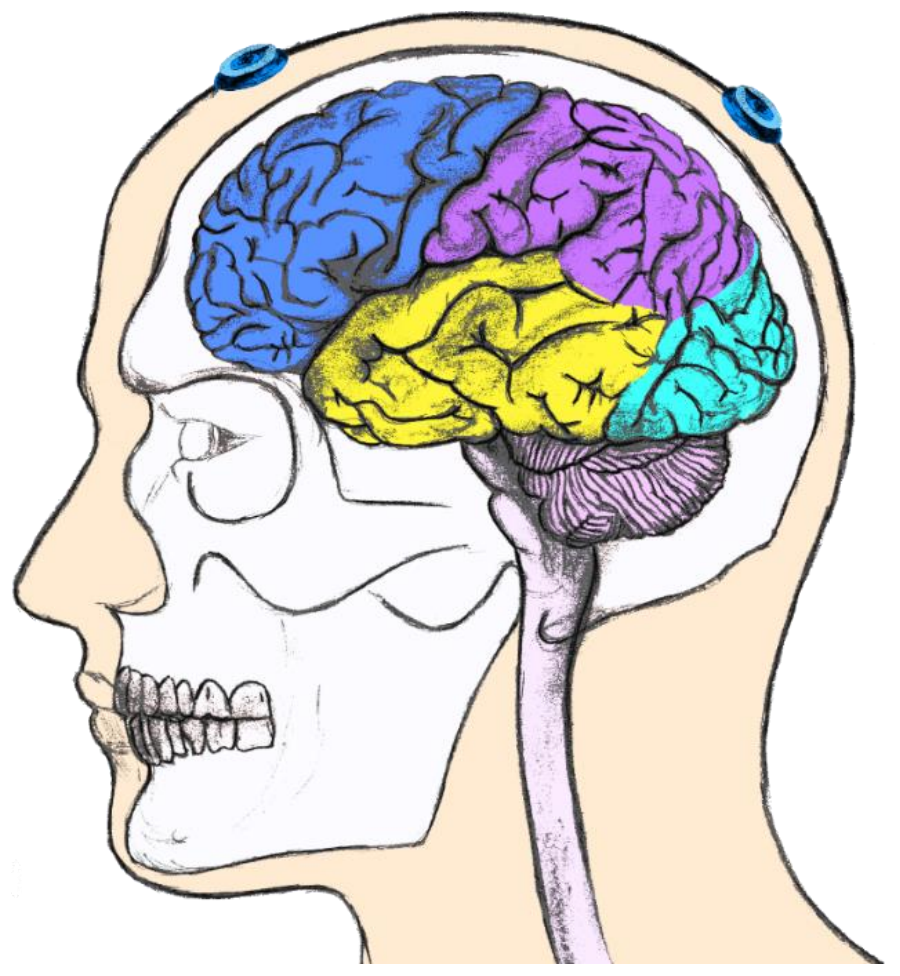
current stimulation (tACS) is a non-invasive investigation method that has been introduced for interference in ongoing brain activity. The method generates weak sinusoidal (alternating) current between electrodes mounted externally on the scalp (see Figure 3 and 4) (Antal and Paulus 2013; Battleday et al. 2014; Vosskuhl et al. 2015b).

Figure 3: Anatomy of the Human Head. The frontal cortex is colored in dark blue and segregated from the parietal cortex (in purple) by the central sulcus. The occipital cortex is marked in aqua. Own illustration (pencil drawing edited with paint.net). 


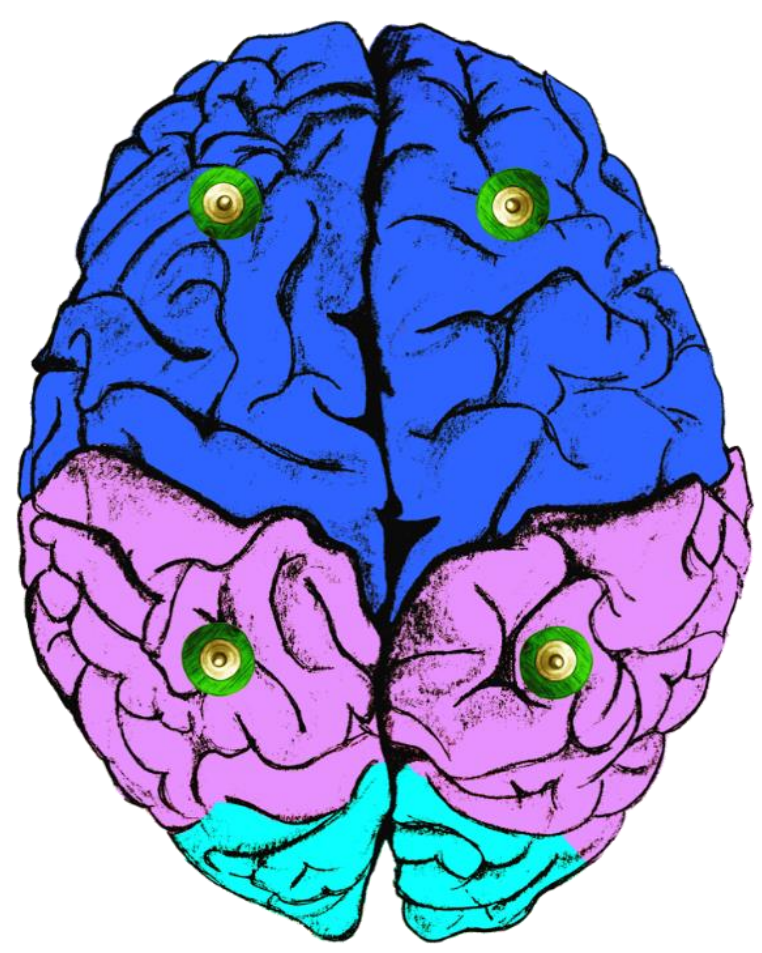

Figure 4: Top View of the Brain. The tACS-electrodes AF3 and AF4 are mounted on the scalp over the frontal cortex (in dark blue) and P3 and P4 are placed over the parietal cortex on the scalp. Own illustration (pencil drawing edited with paint.net).

The net amperage of this rhythmic, sinusoidal electrical current equals zero. Fluctuations of sine waves are created by the alternating current and these fluctuations have been demonstrated to influence endogenous brain oscillations directly (Cohen 2014; Woods et al. 2016): It has been reported that the application of tACS on the scalp affects the electric field of the extracellular space inside of the brain below the threshold, and as a result, to change the transmembrane potential of neurons (Voytek and Knight 2015). Figure 5 depicts how tACS affects ongoing brain oscillations. If tACS is applied frequency-specific, brain oscillations align with the applied phase (Entrainment), or are power modulated (Modulation) (Reato et al. 2013).

\section{Entrainment}

- AC stimulation

- Brain oscillations

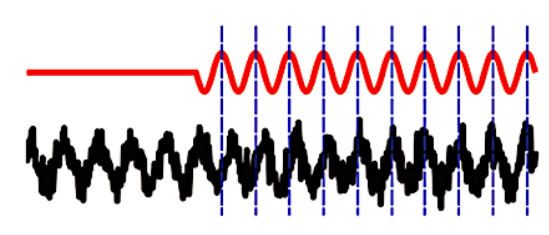

Modulation

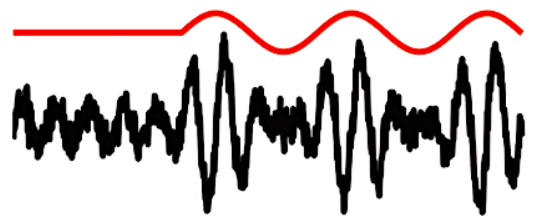

Figure 5: Effects of tACS. A frequency specific phase entrainment and power modulation. From Reato et al., 2013 (with permission). 
This mechanism is assumed to couple a group of neurons by influencing the time coordination and synchrony of their action potential firing, which is entitled as ephaptic coupling (Anastassiou et al. 2011). The firing rate of action potentials is positively related to the stimulation by oscillations. Whereas the quantity in general is not affected over a long time interval, the frequency of the firing rate alternates (Herrmann et al. 2013).

The magnitude of the impact of transcranial stimulation on the brain has been computationally demonstrated using a realistic conductive head model (Holdefer et al. 2006). In this way, the orientation of neurons in the brain has been suggested as substantial for the efficiency of the applied stimulation (Battleday et al. 2014). Current densities in the motor cortex were shown to be significant along white matter fiber tracts if stimulated transcranial. The conductive head model describes anatomically realistic white matter compartments with anisotropies related to resistivity (Holdefer et al. 2006). It was argued that electrical stimulation perpendicular to white matter fiber tracts would result in lower current density, because resistivity is greater along fibers. Recently, studies that combined realistic head models and structural MRI images, have evaluated to what extent non-invasive brain stimulation affects the intracranial current density (Herrmann et al. 2013; Miranda et al. 2013). It has been reported that the current density of $1 \mathrm{~mA}$ effectuates the brain by $0.1 \mathrm{~A} / \mathrm{m}^{2}$, which results in a measurable cortical electric field. In this way, entrainment of brain oscillations influences the neuronal spiking activity (Neuling et al. 2012; Herrmann et al. 2013). In order to have an impact on a targeted brain region, the determined frequency of the AC stimulation has to be close to the actual frequency of the brain oscillations that are responsible for memory (Herrmann et al. 2013; Battleday et al. 2014; Helfrich et al. 2014b; Woods et al. 2016). Furthermore, it has been conclusively shown that effects of tACS are reliant on the phase as well as the brain state (Kirov et al. 2009; Polanía et al. 2012; Neuling et al. 2013; Battleday et al. 2014; Helfrich et al. 2014b). A diversity in phase relations has been suggested for neuronal oscillations (Maris et al. 2016). Phase relations are either ongoing in brain activity or locked to an event. Sensory and motor events have been demonstrated to modulate phase relation diversity, which might play a crucial role in communication among neurons. First, phase relation diversity might lead to the communication between a targeted selection of neurons and a certain network. Second, communication might take place by segregating the flow of information running parallel (Maris et al. 2016). With increasing neuronal activation, a shift in the LFP phase has been observed for gamma band synchronization after introduced visual stimulation. On the contrary, some studies did not report these stimulus-induced shifts (Maris et al. 2016). Phase relation diversity is regarded to be an essential key to decode the firing rate of neurons and to match it to a stimulus configuration. Recently, it has been suggested that the diversity in the theta phase relations in rat hippocampus could 
point to the function of hippocampal neurons that receive information from all sensory modalities in order to determine the position of the animal in place (Maris et al. 2016). Several studies investigated the effects of tACS on brain activity and demonstrated a causal relationship for synchronized brain networks. The electrical stimulation has been reported to directly interfere and manipulate endogenous brain oscillations and, consequently, to modulate brain functions (Antal and Paulus 2013; Herrmann et al. 2013; Battleday et al. 2014; Vosskuhl et al. 2015b; Antal and Herrmann 2016). If sine-wave electric field stimulation can influence human brain networks that conciliate cognition, then this would be observable through an altered behavior (Fröhlich et al. 2015). As stated previously, brain studies have demonstrated that cortical networks are transferring information via synchronizing the neurons in assemblies (Helfrich et al. 2014b), and that the communication of brain networks is feasible through oscillatory activity in the same frequency and with phase coherence (Fröhlich et al. 2015). The exogenous effect of tACS induced rhythmic synchronization of endogenous neuronal activity to facilitate the information transfer within cortical networks, and therefore tACS enhanced cognitive functions. Moreover, tACS has been postulated to affect learning by enhancing the cortical excitability and thus inducing online and offline effects (Antal et al. 2008). In this way, LTP is hypothesized to be induced by online stimulation at the peak of theta oscillations, which has been demonstrated in rat's hippocampus. Offline effects were suggested to arise through lasting impacts of neuroplasticity in stimulated cortical regions, by the adjustment of NMDA receptors and strengthened synaptic contacts. On the contrary, LTD would be induced by stimulation at the trough, which is described as zero-degree- $\left(0^{\circ}-\right)$ phase-lag synchronization. As example of long-lasting effects by tACS, the application of tACS over the motor cortex, for the duration of ten minutes and at the intensity of $1 \mathrm{~mA}$, has been reported to produce effects that last up to an hour after the applied stimulation was terminated (Chaieb et al. 2014). It is to be noted that the application of tACS in the alpha frequency over the motor cortex has been observed to enhance implicit motor learning, yet it did not alternate the EEG power or motor-evoked potentials. As presented for the motor cortex excitability, the inducement of aftereffects represents a frequency-specific effect of tACS (Antal et al. 2008). 


\subsection{Research Hypothesis}

The principle objective of this project was to investigate the physiological structure and connectivity of the WM network in humans. Our research question was whether it is feasible to manipulate the functional connectivity in the fronto-parietal network by using tACS from the perspective of the WM enhancement.

i) First, we hypothesized that the WM network, defined by the bilateral fronto-parietal network between the dIPFC and the PPC, is based on the mechanism of coherence. Moreover, we would find phase-coherence within the theta frequency band in the fronto-parietal network during a WM task performance. The first experiment did not involve stimulation. Instead, EEG was recorded during a WM task for three sessions.

ii) Second, we hypothesized that the WM performance would change by interfering in the theta phase connectivity (in-phase or anti-phase) with tACS-induced oscillations. To show behavioral changes, the WM would improve or degrade according to an enforced synchronization or desynchronization of the WM network. This would be shown by a variation of accuracy rate and reaction time in the WM task performance. In the second experiment, participants followed the same routine while stimulation conditions change during the three sessions.

The goals of the project were first, to establish the EEG biomarkers of the WM performance to validate them to our own task. The connectivity in the theta band of the frontoparietal network illustrates the biomarkers in our WM task. After developing our own biomarkers, we aimed to change the connectivity by applying low intensity, low amplitude, multichannel tACS to affect the WM performance. Previous studies have not attempted to show a causal relationship between the WM of both hemispheres. Last, we evaluated the link between the established biomarkers and WM performance of the subjects. We hypothesized that the oscillatory currents produced by tACS would enhance the WM network by generating greater connectivity between prefrontal and parietal cortexes, and inversely, that disruption of the WM network connectivity would impair the WM performance. 


\section{Methods and Materials}

\subsection{Participants}

Thirty-five healthy and adult volunteers participated in our study. Ten subjects participated in the first experiment ( 6 females, $24 \pm 2.0$ years old, $16.7 \pm 1.8$ years of education (y.o.e.)) and 25 subjects participated in the second experiment (13 females, $23 \pm 2.9$ years old, $16 \pm 2.1$ y.o.e.). Exclusion criteria included neurological or psychiatric disorder and participation in other studies during the last eight weeks. Two participants were excluded from the second experiment due to the nonappearance and they were replaced. After a detailed description of the study, all participants gave written informed consent. The study was conducted in accordance with the Declaration of Helsinki and with permission of the Ethics Committee of the Georg-August University Göttingen (number 23/2/14).

\subsection{Experimental Procedure}

\subsubsection{Experiment 1: Natural History Group}

In Experiment one, ten healthy study volunteers were included in the open-label study for the Natural History Group (NHG). After recruitment, an introductory meeting took place. The participants gave informed consent, were examined neurologically and were familiarized with the WM test. All participants took part in the experiment three times with a 48 hourinterval. During the experiments, the subjects participated in the standardized WM test. The test consisted of two blocks, 100 stimuli each, with two-minute interval between the blocks. All sessions followed the same routine. The EEG was recorded before, during, and after the WM test.

\subsubsection{Experiment 2: Stimulation Group}

Experiment two was a double-blind, placebo controlled, crossover brain stimulation study. It combined multichannel tACS with 8-channel-EEG and the WM task. The methodological procedure followed the above-mentioned routine; however, instead of recording EEG activity during the WM task, tACS was applied. Every volunteer attended three sessions. The stimulation conditions changed between tACS-induced desynchronization (condition A), placebo (condition B) and tACS-induced synchronization (condition C) of the fronto-parietal network. Pre- and post-resting state was recorded for four minutes. 


\subsection{Memory Task Design}

During the experiments, the participants were requested to perform a WM task that specifically demands the three WM phases of stimulus encoding, a maintenance interval and the retrieval of information. The participants completed a 2-back visuo-spatial WM test. It consisted of two blocks with 100 trials per block. Figure 6 depicts the overall task design. Each trial began with the pre-stimulus interval, varied between 1.5 and 2 seconds. Subsequently, the stimulus was presented for $600 \mathrm{~ms}$. The volunteer was supposed to memorize the element presented in either blue or purple and to compare it to the previous stimulus two steps back, given in the same color. After the presentation of the stimulus and a variable post-stimulus interval, a response period (signified with a question mark) appeared. When the question mark was presented, the subject was supposed to press the button on the response box. If the stimulus, e.g. of the blue color matched the previous stimulus in blue two steps back, the participant was instructed to press the green button. If the stimuli did not match in position, the red button was to be pressed.

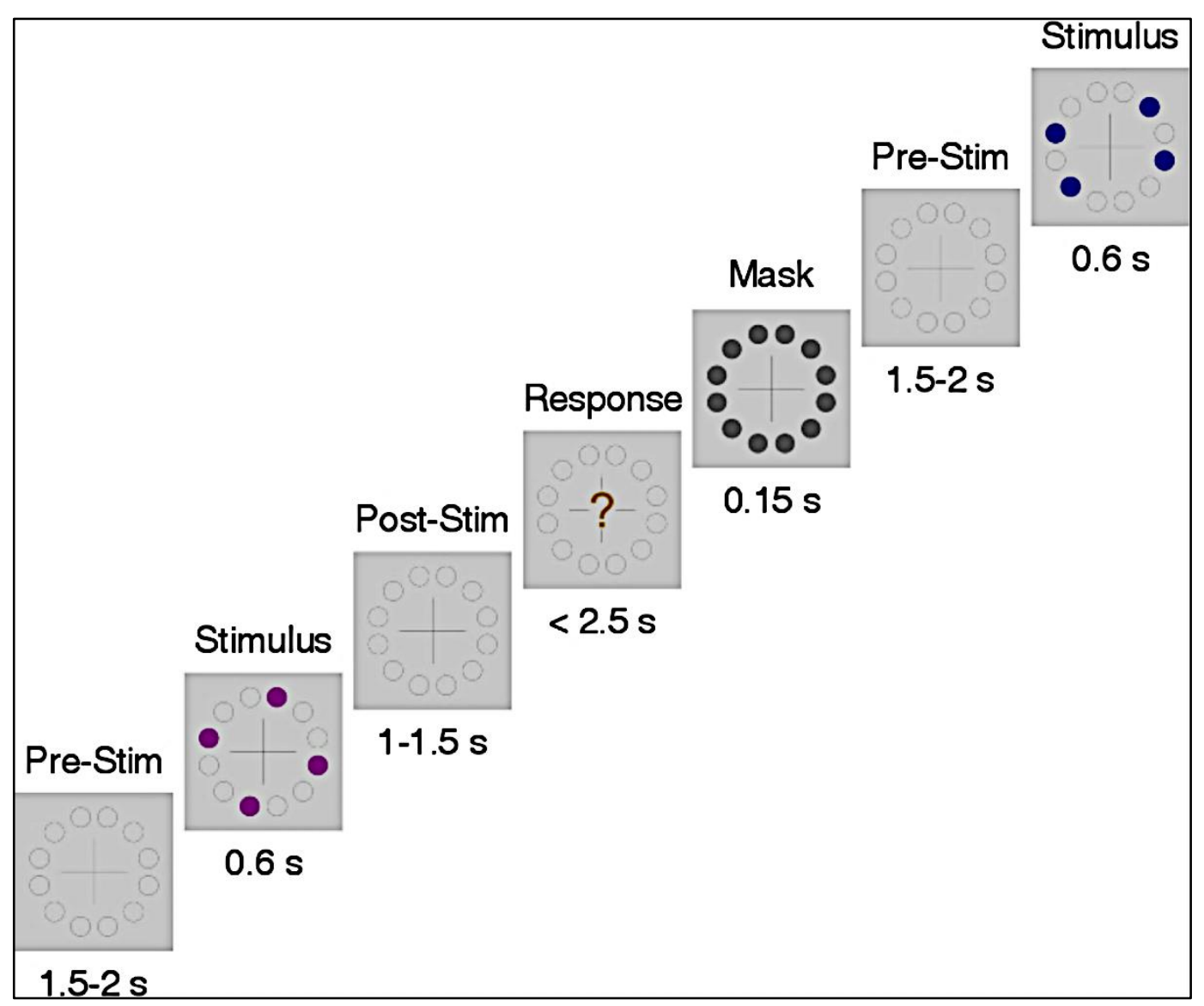

Figure 6: Extract of the 2-back WM Task. Own illustration. 


\subsection{EEG Experiment}

Electrophysiological data were collected in the first Experiment. The high-density 64channel-EEG was synchronized with the computer-assisted WM task. The channels were located according to the international 10-10 system. The recording was conducted with $2 \mathrm{kHz}$ sampling rate and 24-bit digital resolution. All recording was referenced to common average.

\section{5 tACS Experiment}

The method of tACS was delivered in Experiment 2. The condition C intended to synchronize the fronto-parietal network in the anterior-posterior direction and condition A was set the setup for desynchronization. The phase relationships are presented in Figure 7. The conditions, including sham stimulation (condition B), were randomized and counterbalanced across the study. Four electrodes were used for the stimulation over the fronto-parietal network, namely AF3, AF4, P3 and P4 (according to the international 10-10 system. The tACS was delivered for the whole length of the WM task (approximately 18 minutes) with an intensity of 1 $\mathrm{mA}$ peak-to-baseline and the frequency of $6 \mathrm{~Hz}$. Sham stimulation (condition B) was applied by ramping electrical current up for 10 seconds and down immediately after at the beginning and end of every session for Condition B to mimic the starting sensations. The sham stimulation protocol served as our baseline to compare with the stimulation conditions. Figure 7 illustrates the electrode montage for tACS from the aerial perspective. The electrodes, elliptically shaped, are depicted in either purple (anodal electrode) or blue (cathodal electrode) and positioned over the left and right frontal and parietal cortex. The arrows indicate that the alternating current flows back and forth between the electrodes. In the center of the figure, the electrodes are described according to the international 10-10 system. The electrodes AF3 and AF4 are positioned over the left and right dlPFC. The PPC is stimulated by electrodes P3 and P4. The upper part of the figure resembles the setup during stimulation condition $C$, which synchronizes the fronto-parietal network in the anterior-posterior direction. The electrodes over the fronto-parietal cortex are stimulating in-phase, whereas the current over the left and right hemispheres is out-of-phase. The lower part of the figure resembles the stimulation condition A, which desynchronizes the fronto-parietal network in the anterior-posterior direction. Here, the left and right frontal cortexes and bilateral parietal cortexes are stimulated in-phase $\left(0^{\circ}\right.$-phase-lag). At the same time, anterior and posterior electrodes, illustrating the fronto-parietal network, are stimulating out-of-phase. 


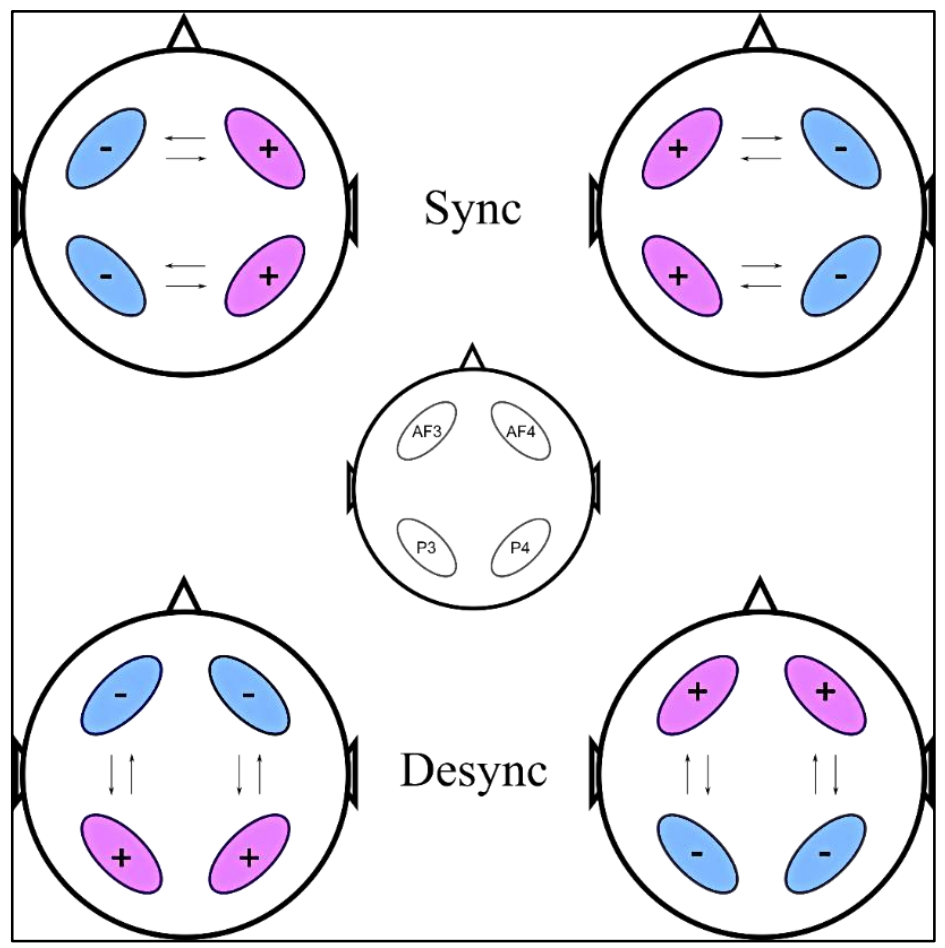

Figure 7: Electrode Montage for tACS. Own illustration.

\subsection{Analysis of Behavioral Data}

During behavioral analysis, the WM performance and reaction time were recorded on the group level. The linear correlation between the session order and the Hit Rate, False Alarm Rate and mean reaction time was analyzed by using the Pearson correlation coefficient. For the preprocessed behavioral data analysis, the focus was on the Performance Rate and Reaction Time. The behavior of the NHG was determined by the WM performance (Hit Rate minus False Alarm Rate) on all three trials.

Table 1 shows an overview of the accuracy terms to describe the subjects' behavior in the WM task. The parameter Hit Rate is calculated by true positive responses divided by the total of true positives plus false negatives. The False Alarm Rate is calculated by false positives divided by false positives plus true negatives. The parameters Hit Rate and False Alarm Rate explain the performance in the WM task.

Table 1: Explanation of the Behavioral Parameters. Own spreadsheet.

\begin{tabular}{|l|l|l|}
\hline & Correct answer "Yes" & Correct answer "No" \\
\hline “Yes" given answer & Hit (true positives) & False Alarm (false positives) \\
\hline “No" given answer & Miss (false negatives) & Rejection (true negatives) \\
\hline
\end{tabular}




\subsection{EEG Data Analysis}

The electrophysiological measurement serves as the method of imaging to evaluate the neurocognitive processes underlying human behavior. Each electrode records activity of neuronal networks which represent oscillations (Cohen 2011; Cohen 2014).

The quantity of electrodes of the EEG recording system was set high (64 channels) for precise localization of ongoing brain activity. Multi-array electrodes increase the accuracy of spatial filters and enable connectivity analysis and topographical distributions (Cohen 2014). The application of electrode gel for every electrode is necessary because air is a poor electrical conductor and gel serves as a conductor by filling in the air pockets (Jackson and Bolger 2014). Before measurement the electrodes are allowed to settle because currents are generated between ions of electrodes and gel. This interaction (half-cell potential) reaches a certain steady state after a few minutes (Jackson and Bolger 2014).

Preprocessing: Skin and scalp muscles are extracellular signals that also generate electric currents and add up to the electrode signal recorded. Signal arising from other sources than the brain contributes to noise, whereas signal relates to the effectively measured voltage of the brain. During data analysis, we are increasing the quality of the signal and therefore increasing the signal-to-noise ratio (SNR) by separating brain activity and extracellular activity (Jackson and Bolger 2014). Since extracellular resources overlie the EEG signal, we applied baseline normalization to focus on task-related dynamics. By preprocessing, background activity is cancelled out and clear data about the participant's brain activity remains (Cohen 2014).

First, we applied a bandpass filter (high and low pass filter). The high pass filter passes signals with a frequency higher than $0.1 \mathrm{~Hz}$ and attenuates frequencies lower than the cutoff frequency (Cohen 2014). The opposite of the high pass filter is the low pass filter, enabling easy passage of frequency signal lower than $125 \mathrm{~Hz}$. Higher frequency is attenuated because it is impeded by differing conductance across brain tissue, the skull and scalp, and the EEG signal is most likely disturbed by artifacts, e.g. noise spikes from pipe lines and eye movements. Second, we down-sampled the originally recorded data at $2 \mathrm{kHz}(2000$ samples each second or two samples each millisecond) to $500 \mathrm{~Hz}$ for faster computation prior to analysis. The temporal resolution of EEG data is determined by the sampling rate, which is defined as recorded EEG sample data per second (Cohen 2014). According to the Nyquist Theorem, the required sampling rate is twice as great as the highest frequency utilized (Omerhodzic et al. 2010; Cohen 2014). Pursuant to the practical limit, we increased signal-to-noise ratio for power and phase predictions by sampling five times the highest frequency we wanted to analyze in the signal. 
Third, detrending organized our data to linear lines, assigning the data to each electrode's origin. Last, we applied Independent Component Analysis (ICA) as a mathematic method to subtract noise and create a high signal-to-noise ratio. The term ICA refers to a computational tool that isolate a signal with multiple variants into separate components "based on the assumption that the components arise from statistically independent non-Gaussian sources" (Menon, 2011, p.484). During $I C A$ we transformed signals to time-series and compared them to uncorrelated activity (sources).

\subsubsection{Preprocessing}

The EEG analysis was performed in MATLAB with the Fieldtrip toolbox. First, the noncausal Butterworth IIR high-pass filter $(>0.1 \mathrm{~Hz})$ and the low-pass filter $(<125 \mathrm{~Hz})$ were applied. The line noise $(50 \mathrm{~Hz}$ and $100 \mathrm{~Hz})$ was rejected with the notch filter. Second, the data was separated on the 2-sec long trials that were time-locked to the onset of the stimulus (Event 1) and response period (Event 2), according to the WM test. Third, the information was detrended and downsampled to $500 \mathrm{~Hz}$ for faster computation prior to analysis. Then trials with abnormal kurtosis were rejected based on the adaptive z-threshold (see Figure 8). We adapted the threshold for each data set to cut off outliners. Variants that are significantly different from the majority were excluded. Finally, $I C A$ was applied to subtract the eye blinking artifacts (see Figure 9).

Figure 8 depicts preprocessing steps for data analysis. The upper part of the figure illustrates the kurtosis per channel per trial. The trial number is entered on the $\mathrm{x}$-axis and the channel number, signifying the electrode number, on the y-axis. The kurtosis is color-coded from low (blue) to high (yellow). The lower figure shows the average kurtosis across all channels per trial. The y-axis of the lower part depicts the kurtosis. Trials with extreme variation in comparison to all other trials were rejected. 


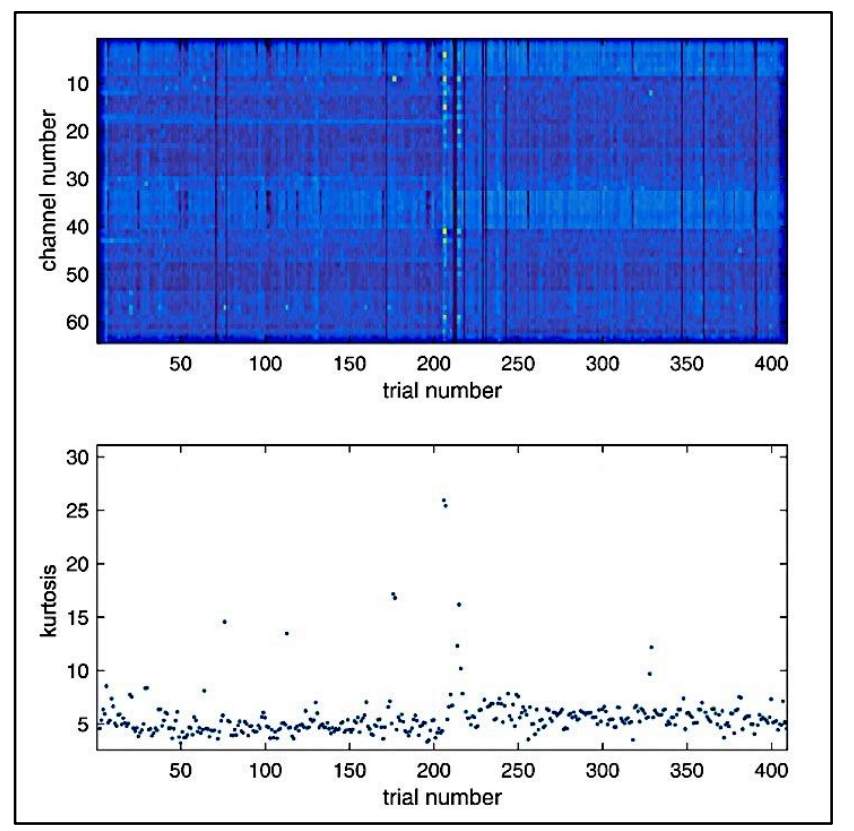

Figure 8: Interface for Trial Rejection based on abnormal Variation of the Data. Own illustration.

Figure 9 illustrates an example of the Independent Component Analysis for the EEG data. During ICA signals are transformed from the sensor to the independent source space. Each trial lasts three seconds. Uncorrelated activity of different sources is displayed. The time course of activity is given on the $\mathrm{x}$-axis and the topology of sources on the $\mathrm{y}$-axis. The round shapes on the y-axis visualize heads in the aerial perspective. The component ica001 is clearly belonging to eye blinking artifacts and can be rejected. Subsequently, the data will be composed back.

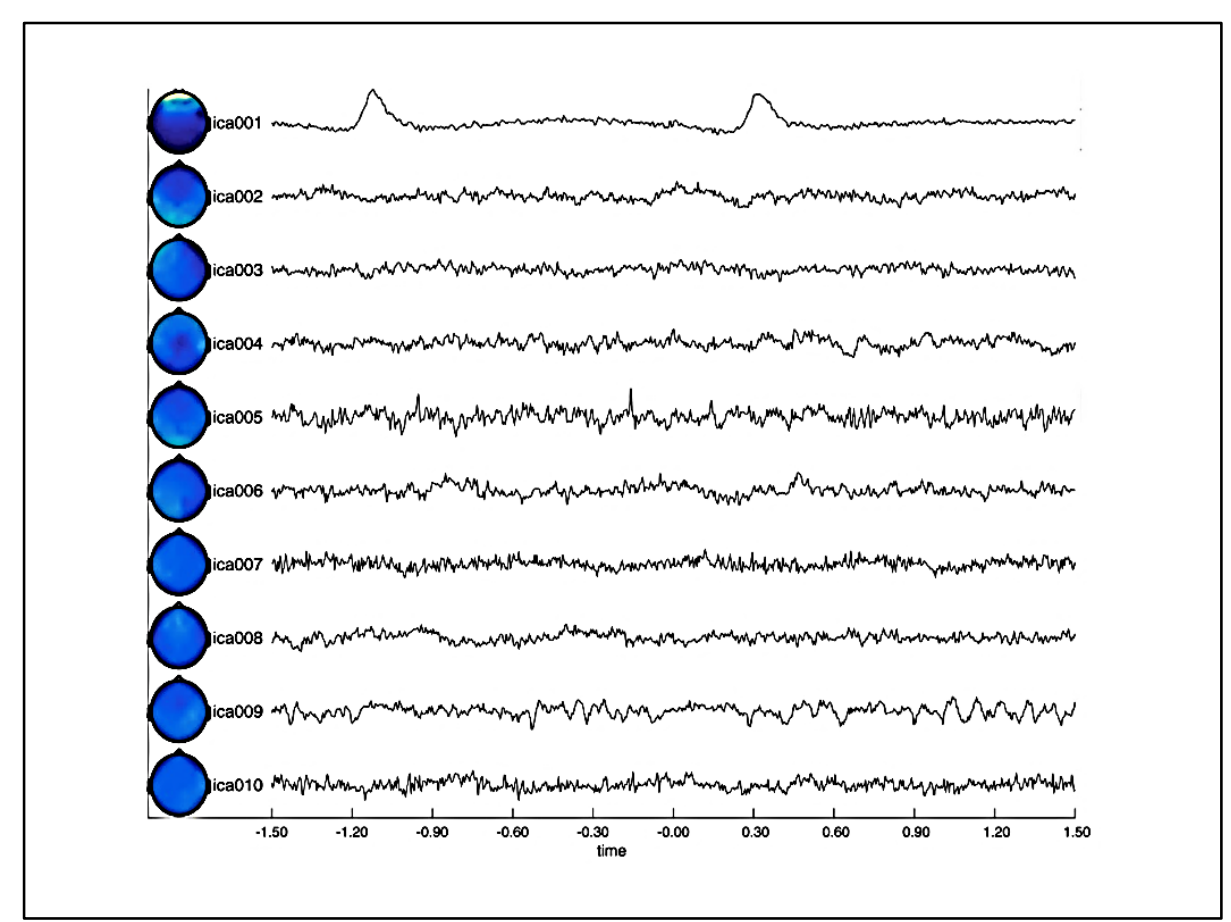

Figure 9: Independent Component Analysis. ICA for EEG channels 1 to 10. Own illustration. 


\subsubsection{Connectivity Analysis}

First, the time-frequency analysis was performed by using the Hanning-tapers. Then the imaginary coberence $(i \mathrm{Coh})$ was estimated for every electrode-pair in the frequency space between 3 to $40 \mathrm{~Hz}$ and in the time interval between $-1 \mathrm{~s}$ to $+1 \mathrm{~s}$ around the event of interest with steps of 50 milliseconds. The thresholded $i \operatorname{Coh}$ (at the level 0.1) was used to build the connectivity graphs. The graphs were computed separately for the theta $(3-8 \mathrm{~Hz})$, alpha $(8-13.5 \mathrm{~Hz})$, beta $(13.5-25 \mathrm{~Hz})$ and gamma $(25-40 \mathrm{~Hz})$ bands. At first, we analyzed the graph density, which is described by a single number from 0 to 1 indicating the proportion of connections (edges) between brain regions (nodes) out of the maximum number of connections. The graph transitivity expresses the level of the clusterization of the electrodes relative to the maximum number of clusters. To establish the significance level, we conducted the permutation test and estimated the confidence intervals (CI) of the spurious coherence. Finally, we plotted the graphs of interest by using the BrainViewer.

\subsection{Brain Stimulation Analysis}

The Analysis of the WM performance and reaction time on the group level was performed with the Bootstrap T-test. The Bootstrap T-Test was conducted to evaluate the behavioral results for the stimulation conditions on the group level. We permuted the data and estimated differences for 10,000 times. The T-Score was calculated for randomly assigned groups. We compared if our real T-score exceeded the $95 \%$ of T-scores distribution of the surrogate data. Furthermore, we applied the Pearson correlation coefficient to analyze the linear correlation of dependency between the conditions for the individual data set. 


\section{Results}

Theta activity is suggested to be necessary for learning processes and active during concentration, attention and the STM activity (Onton et al. 2005). During WM and encoding tasks, theta coherence has been demonstrated between prefrontal and temporo-parietal cortical regions and was associated as a connecting link of widespread brain networks (Kirov et al. 2009). Hence, it could conceivably be hypothesized that we would find phase connectivity in theta oscillations during the memory task. In our first experiment, we aimed to verify the hypothesis by analyzing the $i C o b$ in electrophysiological data. After measuring electrical brain activity, we aimed to perform brain source reconstruction analysis for the NHG. To analyze brain source reconstruction analyzes and for epoching, event markers were sent from the task-discharging computer to the EEG amplifier. The trigger served as labeled time stamps that encode different events in the experiment (Cohen 2014). The purpose of our EEG setting and simultaneous WM task was to demonstrate coherence between frequencies, time and brain regions. After rejecting the ideal coherence, which is most likely due to volume conduction effects, we only consider relationships without ideal coherence. The $i C o h$, defined as the power correlation, estimates the real coherence between recording electrodes. We use this mathematical metric to estimate the relationship between different parts of the fronto-parietal network.

Once the physiological structure of the WM was discovered, we aimed to manipulate the WM network by interfering in the ongoing brain activity by tACS. The second experiment set out to examine the causal role of coherence in the fronto-parietal network. Is coherence rather obligatory for our fronto-parietal network or an epiphenomenon? We investigated the changes on the behavioral stage, by interfering in the coherence via tACS. By manipulating the WM network, this would affect the WM performance, e.g. deteriorate or even improve it.

\subsection{Results of the EEG Analysis}

We first examined the results of the behavioral analysis and concentrated on the dependence of the session order. The behavioral data for the EEG are depicted in Table 2. WM performance score was calculated by Hit Rate minus False Alarm Rate. The reaction time was log-transformed for visual purposes and features the time needed for WM information retrieval and to give an answer on the response box. 


\subsubsection{Behavioral Results}

Table 2 presents the behavioral results obtained from the analysis of the NHG. The mean score of the Hit Rate was $85.2 \pm 1.3 \%$ as the standard error of mean (SEM). On average, the NHG was shown to have scored $13.1 \pm 1.1 \%$ on False Alarm Rate. The WM performance score was $72.1 \pm 1.5 \%$ SEM.

Furthermore, the linear correlation between the session order and the Hit Rate, False Alarm Rate and mean reaction time were analyzed by using the Pearson correlation coefficient. The participants did not improve the Hit Rate by performing the WM task for several times and the reaction time remained similar. Figure 10 depicts the correlation analysis. There was no linear dependence on the session order based on $\mathrm{p}$-value (Hit Rate: $\mathrm{R}^{2}=0.01, \mathrm{p}=0.87$; False Alarm Rate: $\mathrm{R}^{2}=0.08, \mathrm{p}=0.32$; mean Reaction Time $\mathrm{R}^{2}=0.09, \mathrm{p}=0.25$ ).

Table 2: Behavioral Results of the Natural History Group. Own spreadsheet.

\begin{tabular}{|l|l|}
\hline Parameter & Score \\
\hline Hit Rate & $85.2 \pm 1.3 \%($ mean \pm SEM $)$ \\
\hline False Alarm Rate & $13.1 \pm 1.1 \%($ mean \pm SEM $)$ \\
\hline WM Performance & $72.1 \pm 1.5 \%($ mean \pm SEM $)$ \\
\hline Reaction Time & $5.887 \pm 0.031 \mathrm{sec}$ \\
\hline
\end{tabular}
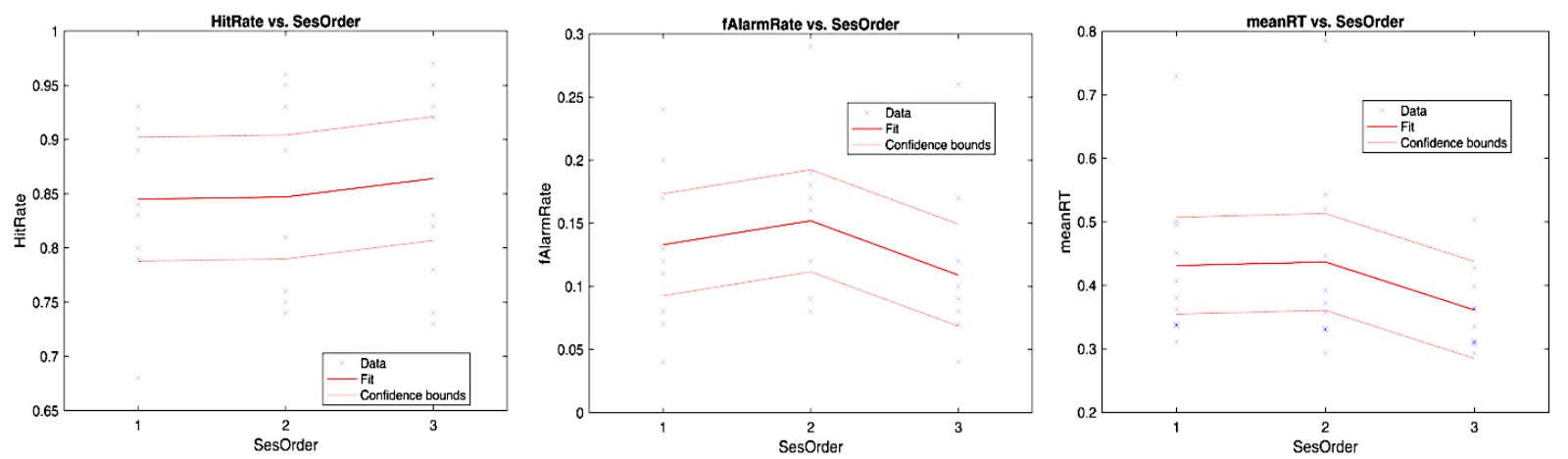

Figure 10: Pearson Correlation Coefficient for the Hit Rate, False Alarm Rate and mean Reaction Time versus the Session Order. Hit Rate: R2 $=0.01, \mathrm{p}=0.87$, False Alarm Rate: R2 $=0.08, \mathrm{p}=0.32$, Working Memory Performance Score (Hit Rate minus False Alarm): $\mathrm{R} 2=0.05, \mathrm{p}=0.24$, Reaction Time: $\mathrm{R} 2=0.09, \mathrm{p}=0.25$. No enhancement or deterioration on the basis of the session order. Own illustration. 


\subsubsection{Results of Graph Density Analysis}

To investigate the EEG phase connectivity of the WM task, we performed graph analysis. The frequencies of interest were separated in frequency bands, from theta to gamma, as listed in Table 3. For each frequency band, the individual upper CI was defined for graph analysis: for density and transitivity. The results of graph analysis are depicted in Figures 11-12. For the first and second event, Figure 11 illustrates the graph density. The figure contains four graphs for event 1 (the stimulus onset) and for event 2 (the response period onset). The time line is given on the $\mathrm{x}$-axis, ranging from $-1 \mathrm{sec}$ to $+1 \mathrm{sec}$ (time interval). The density is plotted on the y-axis. The dashed line illustrates the significance level. The left side depicts event 1 and the right side depicts event 2 for theta density (A for event 1, B for event 2), alpha frequency $(C, D)$, beta $(E, F)$, and gamma frequency bands $(G, H)$. The graph values are listed in Tables 4 and 5 as mean \pm SEM. We applied a second threshold (0.2) to demonstrate that our results are threshold-independent (see Tables 6 and 7).

Table 3: Definition of Frequency Bands and upper Confidence Interval of Density and Transitivity. Own spreadsheet.

\begin{tabular}{|l|l|l|l|}
\hline Frequency Band & Frequency Range & $\begin{array}{l}\text { Upper CI Interval } \\
\text { for Density }\end{array}$ & $\begin{array}{l}\text { Upper CI Interval } \\
\text { for Transitivity }\end{array}$ \\
\hline Theta Band & $3-8 \mathrm{~Hz}$ & 0.178 & 0.184 \\
\hline Alpha Band & $8-13.5 \mathrm{~Hz}$ & 0.160 & 0.171 \\
\hline Beta Band & $13.5-25 \mathrm{~Hz}$ & 0.148 & 0.177 \\
\hline Gamma Band & $25-40 \mathrm{~Hz}$ & 0.187 & 0.246 \\
\hline
\end{tabular}

Table 4: Density, Event 1, "iCoh-” Threshold 0.1 (mean \pm SEM). Own spreadsheet.

\begin{tabular}{|l|l|l|l|}
\hline Frequency Band & Time $\mathbf{- 1 ~ s e c}$ & Time Peak & Time + 1 sec \\
\hline Theta Band & $0.217 \pm 0.017$ & $0.469 \pm 0.026$ & $0.16 \pm 0.021$ \\
\hline Alpha Band & $0.162 \pm 0.018$ & $0.303 \pm 0.029$ & $0.153 \pm 0.018$ \\
\hline Beta Band & $0.13 \pm 0.015$ & $\mathrm{x}$ & $0.117 \pm 0.013$ \\
\hline Gamma Band & $0.117 \pm 0.012$ & $\mathrm{x}$ & $0.123 \pm 0.013$ \\
\hline
\end{tabular}


Table 5: Density, Event 2, "iCoh-" Threshold 0.1 (mean \pm SEM). Own spreadsheet.

\begin{tabular}{|l|l|l|l|}
\hline Frequency Band & Time $\mathbf{- 1 ~ s e c}$ & Time Peak & Time + 1 sec \\
\hline Theta Band & $0.113 \pm 0.013$ & $0.295 \pm 0.027$ & $0.163 \pm 0.019$ \\
\hline $\begin{array}{l}\text { Alpha Band } \\
\text { Beta Band }\end{array}$ & $0.15 \pm 0.019$ & $0.235 \pm 0.022$ & $0.154 \pm 0.017$ \\
\hline Gamma Band & $0.108 \pm 0.01$ & $\mathrm{x}$ & $0.139 \pm 0.017$ \\
\hline
\end{tabular}

Table 6: Density, Event 1," iCoh-" Threshold 0.2 (mean \pm SEM). Own spreadsheet.

\begin{tabular}{|l|l|l|l|}
\hline Frequency Band & Time $\mathbf{- 1 ~ s e c}$ & Time Peak & Time + 1 sec \\
\hline Theta Band & $0.006 \pm 0.003$ & $0.182 \pm 0.02$ & $0.015 \pm 0.0053$ \\
\hline Alpha Band & $0.01 \pm 0.004$ & $0.07 \pm 0.01$ & $0.01 \pm 0.004$ \\
\hline
\end{tabular}

Table 7: Density, Event 2, "iCoh-" Threshold 0.2 (mean \pm SEM). Own spreadsheet.

\begin{tabular}{|l|l|l|l|}
\hline Frequency Band & Time $\mathbf{- 1 ~ s e c}$ & Time Peak & Time + 1 sec \\
\hline Theta Band & $0.003 \pm 0.001$ & $0.076 \pm 0.022$ & $0.012 \pm 0.004$ \\
\hline Alpha Band & $0.01 \pm 0.004$ & $0.028 \pm 0.008$ & $0.01 \pm 0.003$ \\
\hline
\end{tabular}


A

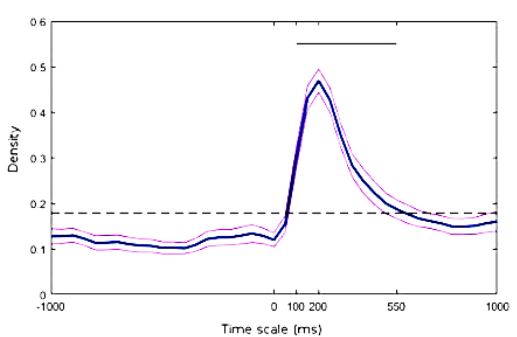

C

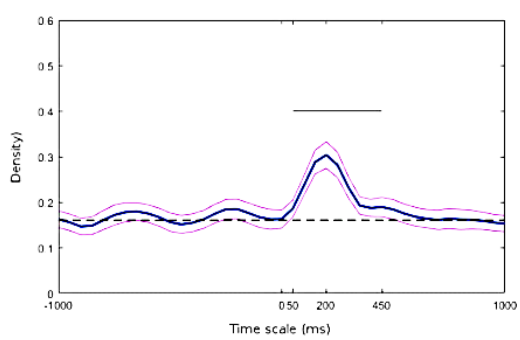

E
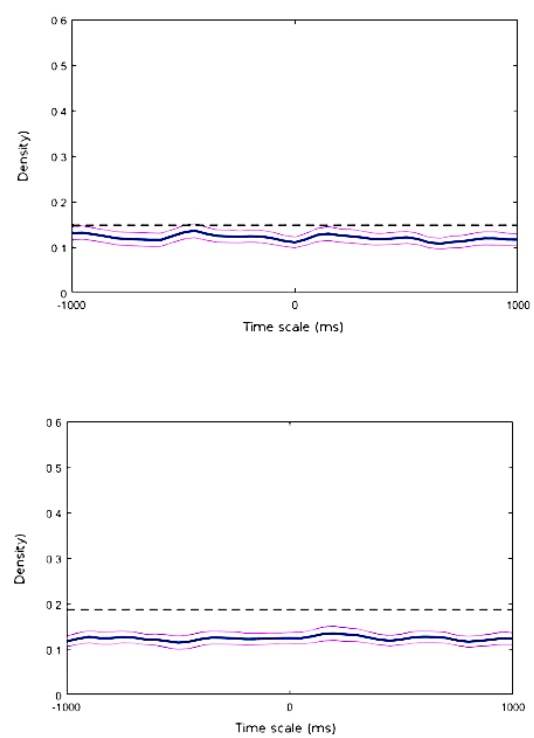
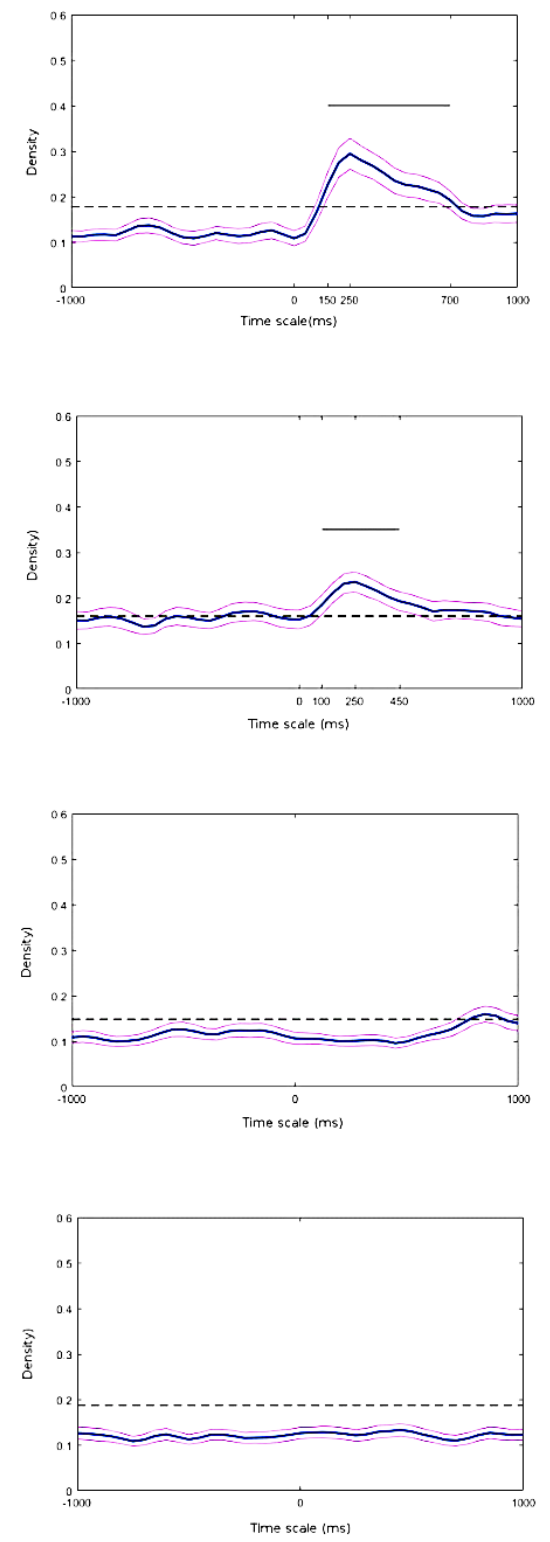

Figure 11: EEG Density of Frequency Bands. Illustration of EEG density for event 1 (on the left) and event 2 (on the right). A and B illustrate theta graph density; C and D depict alpha density; beta density is illustrated in E and F; and gamma frequency is illustrated in $\mathrm{G}, \mathrm{H}$. Theta and alpha density are significantly increased after stimulus onset (event 1 ) and during the response period (event 2). Own illustration.

\subsubsection{Results of Graph Transitivity Analysis}

We analyzed the transitivity, which is described by the level of clusterization. One cluster is made of three nodes and three edges. The level of significance of clusterization is above the upper CI. The threshold was established at 0.1 (10\% of activity). The cluster-based permutation test was used to predict the transitivity. For transitivity, the results were conceptually the same compared to density. Figure 12 depicts the graph analysis for transitivity for event 1 (left side) and event 
2 (right side). The first graphs A and B illustrate the theta transitivity, the second row is the transitivity for alpha $(\mathrm{C}, \mathrm{D})$, the third row depicts beta $(\mathrm{E}, \mathrm{F})$ and the last row is the gamma transitivity $(\mathrm{G}, \mathrm{H})$. Transitivity calculates how many cluster (triangles) we have out of the maximum possible. For our surrogate data, we plotted the upper CI against the real data. The dashed line demonstrates the upper CI (threshold). The activity below the threshold might happen by chance, whereas the situation above the dashed line demonstrates p-value. The solid black line depicts significant clusterization (above the upper $\mathrm{CI}$ ). For theta band, 8 points in time exceed the CI. For the threshold 0.2, we could not find a significant value of clusterization (not shown in the figure). Looking at the Figure 12, it is apparent that the theta band has a significantly higher clusterization than the alpha band. The transitivity was significant for the theta and alpha band. In comparison to the frequencies theta and alpha, the beta and gamma band are diverse (not clusterized). The most interesting aspect of this graph is that the theta band is the highest clusterized graph during Events 1 and 2 . The mean values \pm SEM of the graph analysis are displayed in Table 8.

Table 8: Transitivity, Event 1 and 2, "iCoh-" Threshold 0.1 (mean \pm SEM). Own spreadsheet.

\begin{tabular}{|c|c|c|c|}
\hline Frequency Band & Time -1 sec & Time Peak & Time +1 sec \\
\hline \multicolumn{4}{|l|}{ Event 1} \\
\hline Theta Band & $0.127 \pm 0.016$ & $0.468 \pm 0.027$ & $0.17 \pm 0.021$ \\
\hline Alpha Band & $0.171 \pm 0.02$ & $0.317 \pm 0.029$ & $0.174 \pm 0.019$ \\
\hline Beta Band & $0.160 \pm 0.017$ & $\mathrm{x}$ & $0.128 \pm 0.016$ \\
\hline Gamma Band & $0.139 \pm 0.015$ & $\mathrm{x}$ & $0.1367 \pm 0.016$ \\
\hline \multicolumn{4}{|l|}{ Event 2} \\
\hline Theta Band & $0.129 \pm 0.017$ & $0.304 \pm 0.035$ & $0.165 \pm 0.021$ \\
\hline Alpha Band & $0.16 \pm 0.02$ & $0.238 \pm 0.018$ & $0.169 \pm 0.02$ \\
\hline Beta Band & $0.122 \pm 0.018$ & $\mathrm{x}$ & $0.167 \pm 0.019$ \\
\hline Gamma Band & $0.142 \pm 0.017$ & $\mathrm{x}$ & $0.138 \pm 0.019$ \\
\hline
\end{tabular}




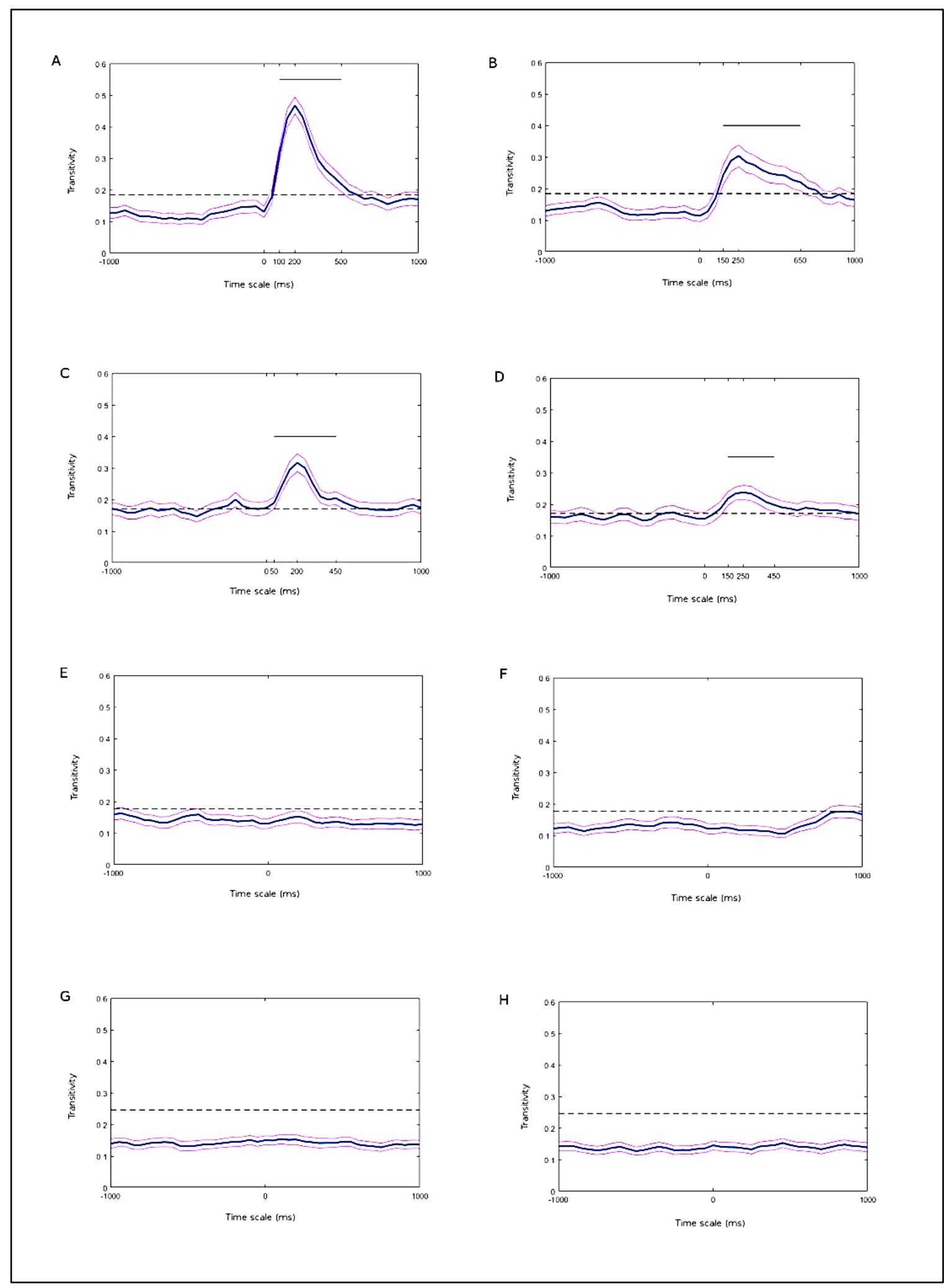

Figure 12: EEG Transitivity of Frequency Bands. Illustration of EEG transitivity during event 1 (on the left) and event 2 (on the right). A, B: theta graph transitivity; C, D: alpha; E, F: beta; and G, H: transitivity of gamma frequency. Theta and alpha transitivity are significantly increased after stimulus onset (event 1 ) and after the response period onset (event 2). 


\subsubsection{Results of Plotted Graph}

Third, we plotted the imaginary coherence and averaged it over all trials. We estimated the mean $i$ Coh between all 64 pairs of electrodes and applied a threshold at $20 \%$ of connectivity (0.2). All values below the threshold were set to zero and above 0.2 were set to one. By doing so, all connections between pairs of electrodes are either one (100\% connections), or zero (none connections). We compared the theta, alpha, beta and gamma bands. On average, the graph densities of Event 1 and 2 (before and after the stimulus onset) were not significant. In addition, the peak of beta and gamma bands did not reach above the threshold. We only found significant connectivity for the peak of graph density for the theta band and relatively smaller significance for the alpha peak. We analyzed only trials with correct responses, because the number of false responses (Miss Rate and False Alarm Rate) was too low.

The results of the analysis are displayed in Figure 13. The figure provides an overview of the plotted $i$ Coh at the maximum density time point during event 1 (depicted on the left) and event 2 (presented on the right). The plotted graphs indicate $20 \%$ of the highest connectivity between recording electrodes at $200 \mathrm{~ms}$ after the stimulus onset (Event 1) and $200 \mathrm{~ms}$ after the response period (Event 2). The peaks of $i$ Coh for theta occurred after $0.468 \mathrm{sec} \pm 0.027$ SEM.
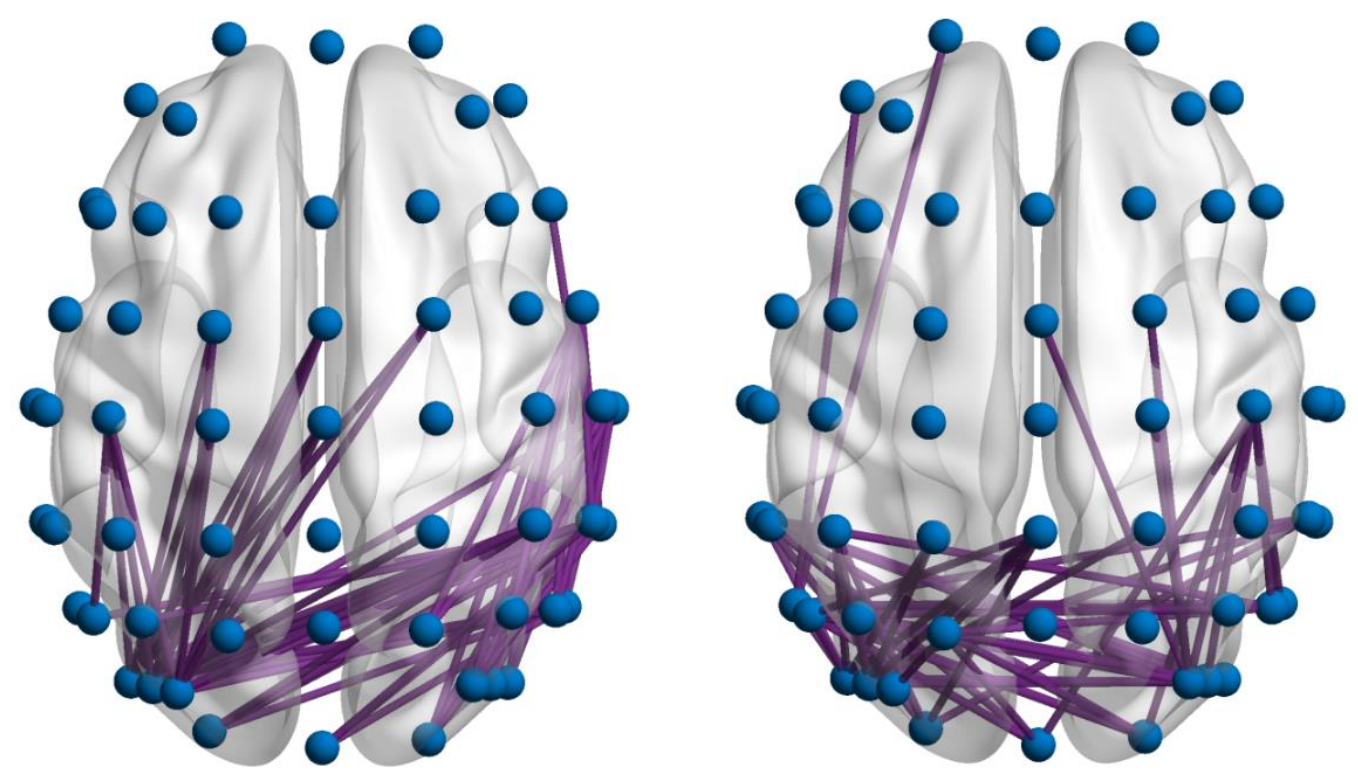

Figure 13: Imaginary Coherence for Theta Peaks. Illustration for events 1 (left) and 2 (right). Event 1 illustrates the stimulus onset, whereas event 2 depicts the response period. Own illustration. 


\subsection{Results of the Stimulation Analysis}

Behavioral results are demonstrated on the group level. Comparisons for the three tACSconditions are made for the WM performance and reaction time. The results of individual data analysis are also depicted.

\subsubsection{Results of Group Data Analysis}

The Analysis of the WM performance and reaction time on the group level was performed with the Bootstrap t-test. The table below (Table 9) illustrates the results for the Stimulation Group for each condition. Table 9 provides the mean \pm SEM of scores for each condition. Condition A is the desynchronization setup, condition B stands for sham stimulation and condition C displays the synchronization of the fronto-parietal network. It can be seen from the data in Table 9 that the mean of the WM score (Hit Rate - False Alarm Rate) was lowest in condition A. In the Bootstrap t-test, the results of WM performance were only significant for condition A compared to conditions B and C (A vs. B: $\mathrm{p}=0.018$ and $\mathrm{A}$ vs. $\mathrm{C}: \mathrm{p}=0.02)$, whereas condition $\mathrm{B}$ and $\mathrm{C}$ did not differ significantly ( $\mathrm{C}$ vs. $\mathrm{B}: \mathrm{p}=0.28)$. Similarly, the reaction time was slowest in condition A (A vs. B: $p=0.015$ and A vs. $C: p=0.049$ ), whereas condition B and C did not differ significantly $(\mathrm{C}$ vs $\mathrm{B}: \mathrm{p}=0.34)$.

Table 9: Scores on Parameters for the Stimulation Group (Conditions A: Desynchronization, B: Sham, C: Synchronization), mean \pm SEM. Own spreadsheet.

\begin{tabular}{|l|l|l|l|}
\hline Parameter & Condition A & Condition B & Condition C \\
\hline Hit Rate & $82.0 \pm 2.5 \%$ & $83.2 \pm 2.4 \%$ & $85.3 \pm 2.2 \%$ \\
\hline False Alarm Rate & $13.7 \pm 1.9 \%$ & $11.9 \pm 1.4 \%$ & $13.3 \pm 1.1 \%$ \\
\hline Overall Performance & $69.0 \pm 2.4 \%$ & $72.8 \pm 2.3 \%$ & $71.9 \pm 2.0 \%$ \\
\hline Reaction Time & $5.93 \pm 0.035 \mathrm{sec}$ & $5.881 \pm 0.030 \mathrm{sec}$ & $5.89 \pm 0.033 \mathrm{sec}$ \\
\hline
\end{tabular}

Figure 14 displays the False Alarm Rate for our tACS-experiment. The graph represents the mean \pm SEM for each condition. The False Alarm Rate, calculated by the false positives divided by the total of false positives plus true negatives (true rejection), displays the percentage of false 'yes' responses (false positives) of the volunteers, indicating that the current and previous stimuli of the same color would match in position, although the real answer was 'no'. All parameters are given as the mean \pm SEM: condition A $(13.7 \pm 1.9 \%), \mathrm{B}(11.9 \pm 1.4 \%), \mathrm{C}(13.3$ 
$\pm 1.1 \%$ ). Condition A represents the desynchronization, condition B is the sham control and condition $\mathrm{C}$ represents the synchronization of the fronto-parietal network.

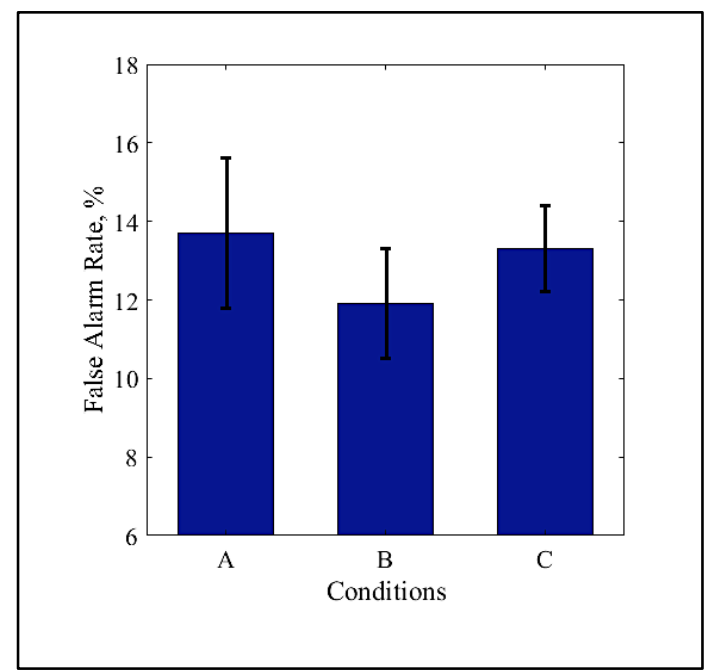

Figure 14: False Alarm Rate as a Function of tACS. Conditions A: Desynchronization, B: Sham, C: Synchronization. Error bars represent mean \pm SEM for each condition. Own illustration.

Figure 15 illustrates the Hit Rate (Condition A: $82.0 \pm 2.5 \%$, B: $83.2 \pm 2.4 \%$, C: $85.3 \pm$ $2.2 \%)$. All values have the mean \pm SEM. The Hit Rate is calculated by true positives divided by the total of all true positives plus false negatives. True positives are 'yes' responses, if the stimulus matched the previous stimulus in the same color. For Condition A, displaying the setup for desynchronization of the fronto-parietal network, the Hit Rate declined compared to Condition B.

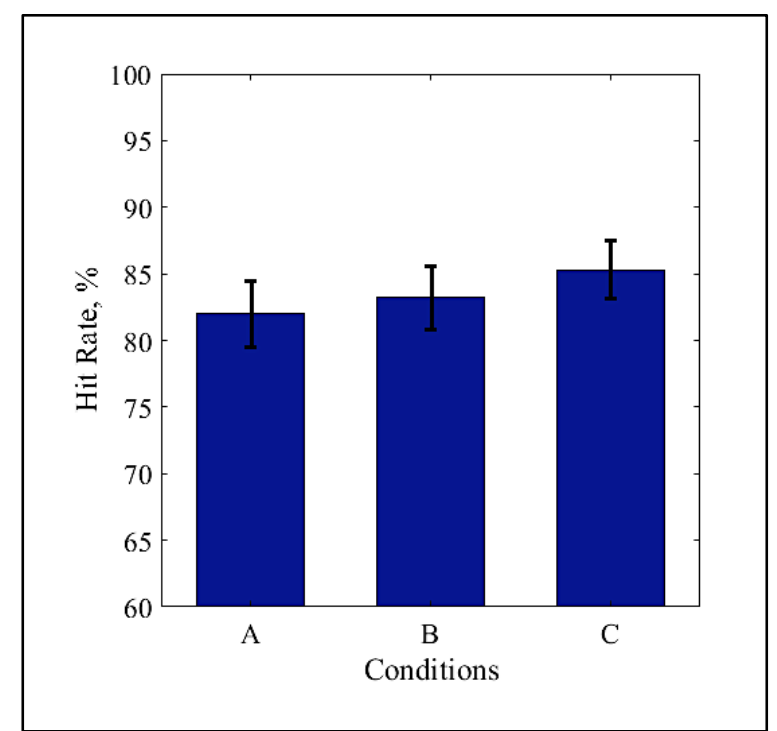

Figure 15: Condition specific Hit Rates in WM Task. The graph illustrates the mean \pm SEM of the Hit Rate for conditions A, B and C. A: Desynchronization, B: Sham, C: Synchronization. Own illustration. 
Figure 16 shows the comparison of the experimental data on the Reaction Times lognormal transformed and for Condition A, B and C (A: $5.93 \pm 0.035, \mathrm{~B}: 5.881 \pm 0.030, \mathrm{C}: 5.89$ $\pm 0.033)$. All parameters are given as the mean \pm SEM. The Reaction Time is defined by the passing time starting from the response period onset and terminated by the 'yes' or 'no' response of the volunteer via response box. For better visualization, the results are log-transformed.

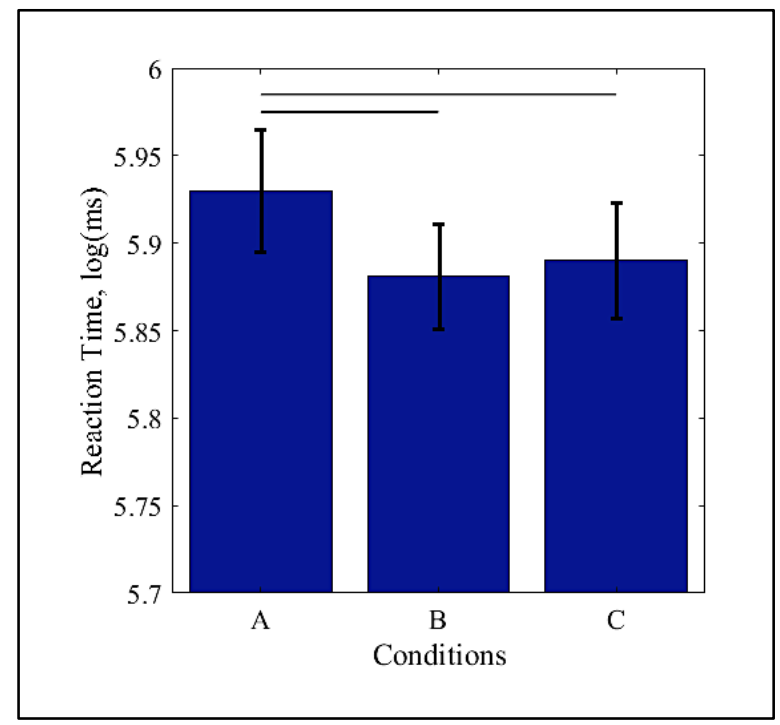

Figure 16: Graph of log-transformed Reaction Time. Error bars represent mean \pm SEM for each condition. A: Desynchronization, B: Sham, C: Synchronization. During active desynchronization of bilateral dIPFC and PPC, the reaction time is slower compared to conditions B and C, versus sham condition, $\mathrm{p}=0.015$. Own illustration.

Figure 17 compares the performance of the volunteers on the WM task for each tACScondition. The graph presents the mean score \pm SEM for the WM performance (Condition A: $69.0 \pm 2.4 \%, \mathrm{~B}: 72.8 \pm 2.3 \%$, C: $71.9 \pm 2.0 \%$. The WM performance is calculated by the subtraction of the False Alarm Rate from the Hit Rate in percent. What stands out in this figure is the difference between condition $\mathrm{A}$ and the other conditions. Whereas condition $\mathrm{B}$ and $\mathrm{C}$ do not differ significantly, the WM performance for condition A is significantly lower compared to sham condition (B) and condition C. 


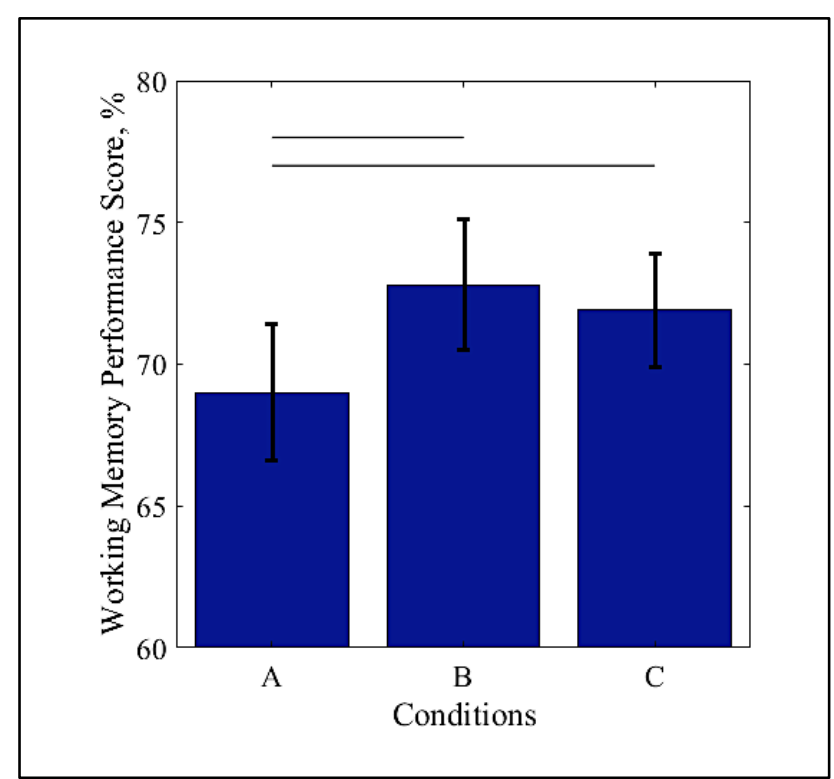

Figure 17: Graph of Working Memory Performance. The WM performance is calculated by Hit Rate - False Alarm Rate. It illustrates mean \pm SEM for each condition. A: Desynchronization, B: Sham, C: Synchronization. For active desynchronization of bilateral dIPFC and PPC, the WM performance is lower for condition A versus conditions B and C, p = 0.018. Own illustration.

\subsubsection{Results of Individual Data Analysis}

The Pearson product moment correlation coefficient was used for individual data analysis, to determine the relationship between two factors: the realperformance in the sham condition and the relative performance under tACS-induced stimulation. The Pearson correlation was calculated by $f x=1+($ Performance Stimulation - Performance Sham $) /$ Performance Sham $)$. As illustrated in Figure 18, it was analyzed whether the stimulation condition (= relative performance) correlates with the WM performance during the control condition (= real performance). A negative correlation was found between the stimulation condition $C$ and sham condition B (Figure 18). No significant differences were found between the conditions for desynchronization (condition A) and control (Condition B) (Figure 19). The graph depicts the WM performance for the sham control versus the synchronization condition. The individual data is illustrated (as crosses). The linear correlation of dependency between sham and synchronization was tested and we found a negative correlation between the WM performance of the control condition and the tACSinduced synchronization. 


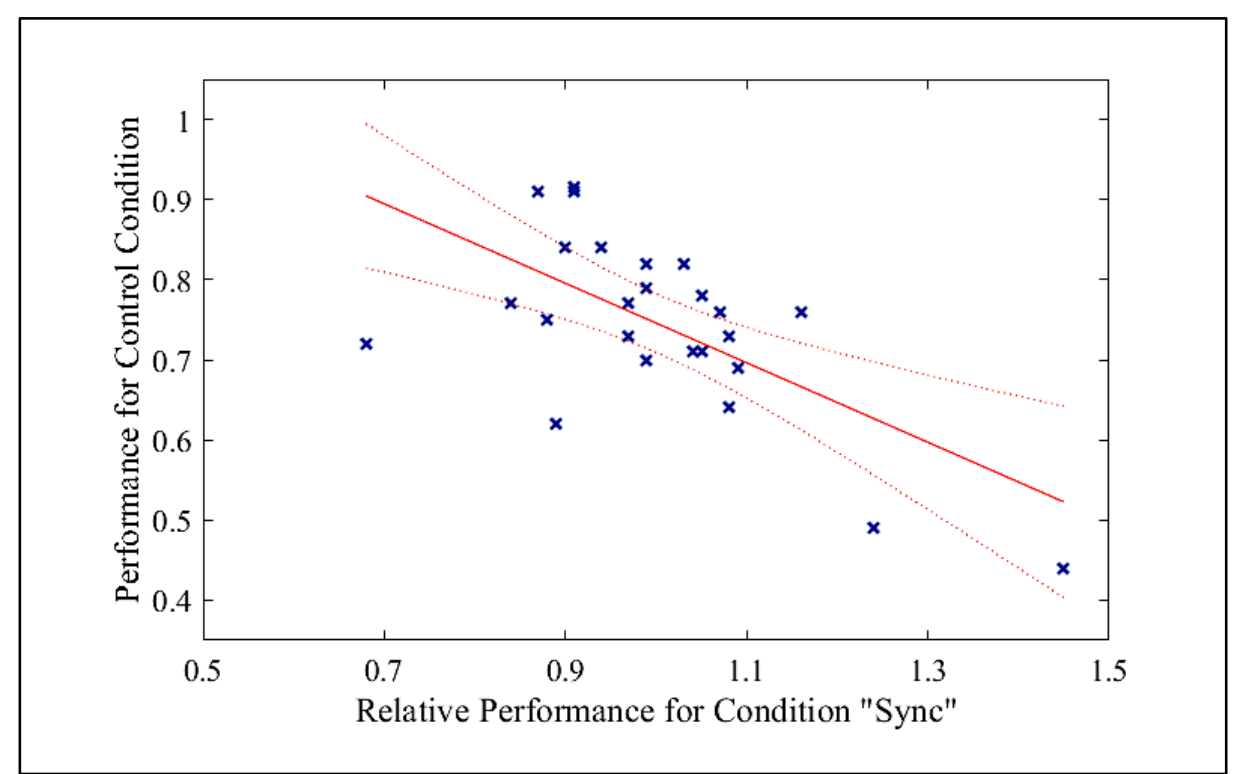

Figure 18: Linear Correlation between Performances for the Control Condition and Synchronization. Individual data analysis. Synchronization vs. control: $\mathrm{p}$ value $=0.0005$, correlation coefficient $=-0.64$. Own illustration.

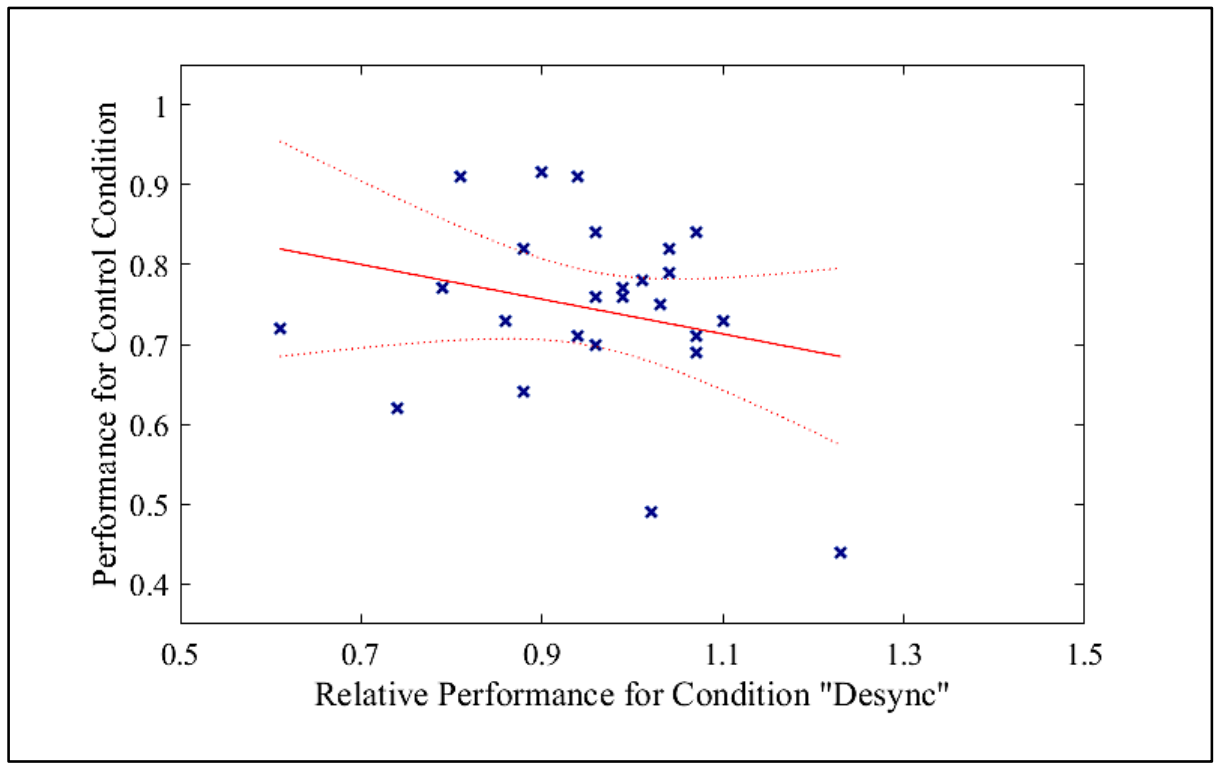

Figure 19: Linear Correlation between Performance for the Control Condition and Desynchronization. Individual data analysis. Desynchronization vs. control: $\mathrm{p}$ value $=0.413$, correlation coefficient $=-0.17$. Own illustration. 


\subsection{Side Effects}

Several studies have postulated the effects of low intensity TES and characterized that it changes the cortical excitability in humans as well as animals (Kirov et al. 2009; Chaieb et al. 2014; Miranda et al. 2013; Jaušovec and Jaušovec 2014; Opitz et al. 2016). Subjects of tACSstudies experienced minor adverse effects such as sleepiness, headaches, skin itching or burning sensation (Neuling et al. 2013; Antal et al. 2017). In comparison, the application of tACS has been reported to provoke retinal phosphenes or flashes in some participants, which is most likely produced by direct stimulation of the eye (Herrmann et al. 2013). It has been argued, that this adverse effect may lead to unblinding of the subject (Chaieb et al. 2014). Therefore, we applied sinusoidal current also during the sham condition for a short period of time in the beginning and end of the session to provide for blinding of all subjects. Nevertheless, sham stimulation does not induce alternations in the cortical excitability and serves as a reference. Furthermore, we applied tACS in a frequency range which is less likely to provoke phosphenes than the maximum between frequencies of 10 and $20 \mathrm{kHz}$, but more likely than higher frequencies. Additionally, two stimulation setups (synchronization and desynchronization) were chosen to make it less likely for subjects to guess which stimulation technique is applied. One subject reported about phosphenes prior to the task beginning. No adverse effects related to skin sensations such as burning were registered. 


\section{Discussion}

This work investigated the physiological structure and connectivity of the WM network in healthy humans. The main aim of the first experiment was to demonstrate theta coherence within the WM network between the dIPFC and the PPC. The second experiment was designed to test the feasibility of the manipulation with the WM network. The overall aim was to show behavioral changes due to synchronization or desynchronization of the WM by applying tACS using $6 \mathrm{~Hz}$ as the stimulation frequency over the bilateral fronto-parietal network. Therefore, we determined to what extent transcranial stimulation affects the human brain.

The first hypothesis was that the WM network is based on the mechanism of coherence. In the first experiment, the subjects participated in three sessions. During each meeting, they were engaged in a 2-back task and EEG was recorded during pre- and post-resting state and simultaneous with the task processing. The research question was first, whether the theta frequency significantly increased during WM task processing and second, if multiple processing of the 2-back task would improve the WM Performance or even accelerate the reaction time. Preresting state served as the baseline for subsequent analysis.

The second hypothesis was that tACS would interfere with ongoing theta coherence and that this would be shown by an alternation in the WM performance. A similar methodological approach was followed for the second experiment, as in the first one. However, instead of recording EEG during task processing, tACS was introduced. An alternating electric field was applied across the conductive electrodes AF3, AF4 (targeting the left and right dlPFC) and P3, P4 (targeting the bilateral PPC) with an intensity of $1 \mathrm{~mA}$ peak-to-baseline using an upper theta frequency $(6 \mathrm{~Hz})$. Additionally, EEG was recorded during pre- and post-resting state. The subjects also participated in three sessions but, in contrast to the first experiment, the conditions varied. Three experimental conditions (randomized across the subjects) consisted of first, the bilateral desynchronization of the dIPFC and PPC (condition A), second, the Sham condition (B) and third, the synchronization of the dPPFC and PPC bilaterally (condition C). During synchronization of the fronto-parietal network, the left and right hemispheres were desynchronized at the same time and vice versa.

The EEG-experiment provided for general results in behavioral analysis and accurate results in graph density and transitivity as well as plotted graph analysis. Behavioral results presented the WM performance for three sessions. Relationships in oscillatory frequencies were estimated between different parts of the bilateral fronto-parietal network to investigate the physiological structure of the WM in humans. The tACS-experiment analysis comprised results for data analysis on the group level and individual data analysis by evaluating the effects of tACS over the fronto-parietal network that supports the WM. 


\subsection{Domain of Technology}

In the domain of electrophysiological technology, other studies discovered what underlies the recorded EEG signals. The EEG measurement captures the amplitude and phase of neuronal oscillations and this is thought to reflect mental processes (Sauseng et al. 2006; Srinivasan et al. 2006; Herrmann et al. 2016). The recorded EEG signal constitutes of various, superimposed frequencies that can be separated for analysis. The frequencies are assumed to reflect information transfer among nerve cells (Watson and Buzsáki 2013). Human studies examined EEG data and found phase synchronization of oscillations between two brain regions or an augmentation of intra-regional oscillation power, which presumably originated from synchronized postsynaptic potentials of groups of neurons (Fell and Axmacher 2011).

The focus of the first experiment lies on EEG connectivity. In this perspective, previous studies have already led to important conclusions about the WM connectivity (Polanía et al. 2012; Kawasaki et al. 2014; Voytek and Knight 2015). Synchronization of oscillations has been claimed to be crucial for many cognitive processes, including memory formation (Fell and Axmacher 2011). In fact, a communication mechanism and information flow within the WM network and related brain areas has been suggested to underlie phase synchronization in the theta frequency. In our assumption, the dIPFC interacts with the PPC using this frequency. It has been observed that phase-synchronization indeed takes place between the fronto-parietal regions (Palva et al. 2010). Additionally, theta band activity was recorded in rats, between subcortical structures, including the hippocampus and amygdala (Onton et al. 2005). More precise, previous research has established that phase-synchronization in the theta frequency $(6 \mathrm{~Hz})$ takes place in the fronto-parietal network during the WM processes of encoding, maintenance and retrieval in auditory-verbal or visual WM tasks (Kawasaki et al. 2014). It was further revealed that theta coherence selectively engages and links brain areas for WM task processing (Kawasaki et al. 2014). During WM task performance, increased theta coherence was demonstrated in the fronto-parietal network. Theta coherence was interpreted as the working unit which is required for spatial WM tasks and simultaneously, synchronization of the fronto-temporal network has been reported during visual WM tasks.

To provide causal evidence for the effects of transcranial stimulation on human behavior, we targeted the WM network. As discussed above, the WM brain network is detected in the fronto-parietal cortices and subcortical structures. Only superficial cortical areas can be reached by the generated current of stimulation electrodes, since current density decreases with distance. Thereby, solely the fronto-parietal cortex areas, underlying WM, were stimulated directly. Nevertheless, stimulation might also influence subcortical structures indirectly by oscillatory communication via PAC. We assume that the hippocampal formation is indirectly affected by tACS 
because it is revealed to be part of the memory formation and thus also part of the WM network (Onton et al. 2005). The second experiment intended to manipulate EEG connectivity by tACS. Recent studies investigated that tACS interacts with endogenous network dynamics (Fröhlich and McCormick 2010; Neuling et al. 2013). By applying an alternating field, entrainment is thought to occur by influencing the phase of ongoing brain oscillations in a frequency-depended matter (Fries 2005; Reato et al. 2013; Helfrich et al. 2014a; Voytek and Knight 2015). Entrainment of focused brain areas might imitate top-down control as has been suggested for interhemispheric synchronization in the gamma-band frequency (Helfrich et al. 2014b). What is the neuronal mechanism behind synchronization? Previous research described that phase synchronization of oscillations between different brain regions would contribute to the accuracy of neuronal spike timing, which is relevant for neural communication and synaptic plasticity (Uhlhaas and Singer 2010; Fell and Axmacher 2011). In animal studies, synaptic plasticity has been demonstrated for theta phase coupling (Fell and Axmacher 2011). When networks are synchronized, the oscillations would align in phase and amplitude (mechanism of coherence) and therefore the probability of neuronal spiking would increase. The interregional oscillatory activity in turn could be measured by an oscillatory power increase (modulation) in EEG recording (Reato et al. 2013).

Regarding non-invasive brain stimulation, several previous studies concentrated on the improvement of human WM by using tACS over the cortical areas (Pasternak and Greenlee 2005; Polanía et al. 2012; Jaušovec and Jaušovec 2014; Kawasaki et al. 2014; Violante et al. 2017). Kawasaki et al. (2014) have causally linked theta phase synchronization between the frontoparietal cortexes with auditory-verbal and visual WM processes (Kawasaki et al. 2014). Whereas they applied current in the same theta frequency $(6 \mathrm{~Hz})$, a different electrode setup was defined (AF3, P5, PZ electrodes) to record theta coherence. Furthermore, Polanía et al. (2012) stimulated the left fronto-parietal WM network in the theta range (6 Hz) (Polanía et al. 2012). Similarly, a recent study by Violante (2017) targeted the fronto-parietal network by in-phase and anti-phase theta stimulation (6 Hz tACS) over the right hemisphere (Violante et al. 2017). It was argued that long-range networks would function by synchronization of oscillatory brain activity (Violante et al. 2017). Respectively, it is suggested to be the key mechanism for the WM network. Both studies claim that the synchronization would be induced by tACS in the theta range and that this affected the WM performance of the subjects positively (Polanía et al. 2012; Violante et al. 2017). Furthermore, they suggest that anti-phase tACS over the left or right fronto-parietal network impaired the WM performance. Interestingly, fMRI data recorded during the stimulation revealed that the effect size was related to the relative phase of the stimulation and that it alternated due to the internal cognitive processing state (Violante et al. 2017). Violante et al. 
(2017) claimed that the application of tACS with $0^{\circ}$-phase-lag between F4 and P4, thus the electrodes were acting in synchrony, caused a higher effect size, whereas the desynchronization of F4 and P4 with $180^{\circ}$-phase-lag led to a lower effect size. These effects were only reported for tasks with high demands on the subjects' cognitive skills.

\subsection{EEG Interpretation}

An initial object of the first experiment was to find support for the hypothesis that coherence plays a causal role in the fronto-parietal WM network. The WM of subjects was targeted by the 2-back task and measured ongoing brain activity by EEG. We aimed for an exclusion of the ceiling effect in our behavioral data by choosing a difficult WM task design. The behavioral results (see Results section) indicated that there is no ceiling effect, since the WM performance is averaged (mean \pm SEM were $72.1 \pm 1.5 \%$ ). The sessions were repeated for three times and correlation analysis did not show significant effects based on the session order.

On the question of EEG frequency analysis, this study investigated EEG graph density. The most interesting finding is that theta and alpha density are significantly exceeding the threshold in both events (A-D), whereas, on the contrary, no increase in beta or gamma density is detected. What stands out in Figure 11 is the dominance of theta density (see A, B). A possible explanation of this might be that theta frequency significantly increased during WM encoding (stimulus onset for event 1) and retrieval (response period onset for event 2). Depicted on the time scale in A, 200 milliseconds after stimulus onset, theta density reaches the maximum. This observation further supports the ideas of theta connectivity in long-range networks (Sauseng et al. 2005; Palva et al. 2010) and that large areas of the brain are activated during task processing.

Furthermore, transitivity was analyzed for the frequency bands theta, alpha, beta and gamma. Results only demonstrated significant clusterization for the theta and alpha frequencies (see for events 1 and 2 in A-D), but not for the other frequency bands, as described for graph density. It is striking that for theta, the clusterization is higher compared to alpha. Moreover, event 1 shows greater transitivity compared to event 2 (see A compared to B, C and D). These results are conceptually the same compared with the graph density and may be explained by the assumption that theta and alpha activity are crucial for communication between brain areas during WM processes (Sauseng et al. 2005; Sauseng et al. 2006; Griesmayr et al. 2014).

Figure 13 shows a strong connectivity between the occipital cortex and the parietal cortex, which indicates a strong occipito-parietal network activity. We assume that this reflects processing of visual information during stimulus onset. The encoded stimulus information flow would be transmitted from the visual cortex to the parietal cortex. At first, visual cortex areas 
V1 and V2 receive information about the direction of the stimulus. Second, V4 and V5 process information about the abstract visual process. Afterwards, the visual information flows to the storage area, which is illustrated by the parietal cortex. Contrary to our expectations, this study did not find maximal theta coherence between the frontal and the parietal cortexes. We argue that the information flow would afterwards continue to the fronto-parietal network, although we could not plot the activity directly. These results confirm the association between theta coherence and WM functions. We found a significant increase in theta activity, as well as alpha activity, in occipital areas. In accordance with neuroimaging studies, the alpha frequency may display visual information processing by alpha coherence, whereas theta coherence might account for attentional demands in the frontal region (Griesmayr et al. 2010; Griesmayr et al. 2014). After maintenance of visual information, the frontal and parietal cortex are assumed to cooperate in the fronto-parietal WM network for manipulation of stored information. The prominent theta activity may represent the activation of scopes of WM by the CE (Sauseng et al. 2005; Palva et al. 2010).

Taken together, these results support the idea that the WM network is based on the mechanism of theta coherence. With respect to the first research question, theta frequency increased during task processing and regarding the second research question, the session order had no significant effect on the WM performance or reaction time.

\section{3 tACS Interpretation}

After developing the biomarkers showing theta phase connectivity, we aimed to modify the WM performance by affecting theta brain oscillations. To give conclusive evidence of a causal relationship between WM activity and electrical stimulation, the manipulation via tACS must change ongoing oscillations of the subject completing a task specific for WM performance. We chose a multi-electrode configuration (AF3, AF4, P3, P4) that enables a focused stimulation of the bilateral WM network (Helfrich et al. 2014b). Moreover, the application of small electrodes was suggested to improve focalization of the stimulation (Miranda et al. 2013). In the tACS experiment, the outcomes for the conditions A (desynchronization), B (sham stimulation) and C (synchronization) were first compared on the group level and finally on the individual level. The average log-transformed reaction time was significantly different for the desynchronizing condition versus the sham condition or versus the synchronizing condition (A vs. B: $\mathrm{p}=0.015$, A vs. $\mathrm{C}: \mathrm{p}=0.049)$. The observed increase in reaction time could be attributed to the active desynchronization of bilateral dIPFC and PPC. Additionally, the WM performance score is significantly lower for condition A compared to conditions B ( $\mathrm{p}=0.018)$ and $\mathrm{C}(\mathrm{p}=0.02)$. It is 
possible that active desynchronization of the fronto-parietal cortexes impaired the WM performance of the subjects. In contrast, no significant modification was found for WM performances for conditions $B$ and $C(p=0.28)$ or for the reaction time analysis for conditions sham and synchronization $(\mathrm{C}$ vs. $\mathrm{B}: \mathrm{p}=0.34)$.

This outcome is contrary to findings found by Violante et. al. (2017) and Polanía et al. (2012) who claimed that synchronization over the left (Polanía et al. 2012) or right hemisphere (Violante et al. 2017) could enhance the WM performance and reduce the reaction time. In agreement with our findings, the studies demonstrated WM impairment due to the condition for desynchronization of the fronto-parietal network. However, major differences can be found for the in-phase stimulation (condition C). This inconsistency may be due to a slightly but substantially different tACS application set up. On the one hand, the same frequency $(6 \mathrm{~Hz})$ was chosen over the same brain area (fronto-parietal), but on the other hand, Polanía et al. (2012) positioned the stimulation electrodes over the left hemisphere of right-handed subjects whereas we stimulated the bilateral cortex and therefore included the right hemisphere. By choosing a bilateral study set-up, we did not have to exclude left-handers from the study.

What is the neuronal mechanism behind impairment due to the decoupling of the frontoparietal brain network? Data from animal studies suggested that NMDA receptors are the target for long-range synchronization of local circuits (Uhlhaas and Singer 2010). Hence, it could conceivably be assumed that desynchronization interfered with a LTD-like effect. Synaptic contacts would be loosened and less information could be transferred in a slower speed.

Although we did not find an effect of the synchronization condition versus sham control on the group level, the analysis of the individual data revealed important findings. For individual data analysis, the real performance in the sham condition was opposed to either the relative performance under tACS-induced synchronization or the condition for desynchronization. No significant differences were found for the linear correlation between performances for the control condition and desynchronization (see Figure 19). Nevertheless, there was a significant negative correlation between the two conditions B (sham) and C (synchronization). There was an improvement of WM performance for participants who scored low during the sham condition. On the contrary, participants with high WM performance during sham control scored lower during the synchronization condition. These findings are surprising, and we may speculate that this effect cancelled out a significant difference between the control and synchronization condition. These results indicate that subjects, who scored above average in the sham condition, were negatively affected from the synchronization condition. Inversely, participants with poorer results for sham scored higher during synchronization of the fronto-parietal network. Taking together, it can be suggested that we did not find significant results in the overall design for the 
synchronization condition compared to sham, because the contradictory results of the stimulation on high and low performers cancelled out a significant effect. As described earlier, a balance in synchronization and decoupling is suggested as mandatory for healthy brain development and functions (Voytek and Knight 2015). On the one hand, good WM performers scored lower during the synchronization condition. It can be speculated that good performers already have a well-optimized network and this may be impaired through interference. On the other hand, participants who scored lower during the sham condition, improved with the tACS-induced synchronization. Therefore, the tACS-induced synchronization supposedly improved the WM of performers with a less optimized network. In accordance with the present results, previous studies have demonstrated that synchronization has the tendency to improve a less optimized WM network and to impair a well-optimized network (Holdefer et al. 2006; Voytek and Knight 2015; Violante et al. 2017). Our findings are in line with the theory of Voytek and Knight (2015), claiming that well optimized oscillatory coupling between brain areas is essential for successful and coordinated neuronal activity (Voytek and Knight 2015).

Previous studies intended to manipulate endogenous brain activity (Neuling et al. 2013; Vossen et al. 2015; Santarnecchi et al. 2016). Neuling et al. (2013) were solely able to enhance low endogenous alpha power whereas subjects with high alpha power could not be altered (Neuling et al. 2013). By this, frequency-specific tACS has been shown to reduce individual differences in the WM performance. In our study, the healthy participants that reached high accuracy and performed the task by short reaction times probably cannot be improved further. On the one hand, they might already have reached their individual maximum of endogenous theta power and this may not be improved, but on the other hand, their WM performance might be deteriorated by over-coupling or under-coupling, as shown in the results for the desynchronizing condition. Contrarily, subjects with inferior performance have been shown to be enhanced by tACS. As a result, we speculate that patients with altered theta power may perhaps benefit from the theta-tACS stimulation.

A study conducted by Meiron and Lavidor (2014) reported an enhancement of the cognitive control function during a verbal WM task by applying tACS in the theta frequency (Meiron and Lavidor 2014). They applied an oscillating current of $4.5 \mathrm{~Hz}$ over the prefrontal area and targeted the dIPFC bilaterally to enhance the verbal WM while the subjects were occupied in the WM task during and after tACS. After the event, the participants were asked to evaluate their outcome in the WM tasks in order to compare the WM accuracy and correlate it to the self-perception of the participants. After analyzing the results of this study, they concluded that the dIPFC would be engaged in remembering the WM performance. Interestingly, they claimed that tACS interfered in ongoing oscillatory activity and alleged that it would be 
feasible to treat the theta related cognitive dysfunctions in schizophrenic patients (Meiron and Lavidor 2014). Impaired oscillatory brain activity has been reported for diseases such as altered EEG frequency in the beta range in Parkinson's disease and the dysregulation of gamma activity in patients suffering from schizophrenia or ADHD (Uhlhaas and Singer 2010; Neuling et al. 2013). In comparison to our study, the dlPFC was also targeted bilaterally by Meiron and Lavidor (2014). Analytical procedures were verified by online WM tasks and post-stimulation tasks. Conversely to the visuo-spatial WM, they intended to enhance the verbal WM. We may speculate that tACS applied in the individual EEG frequency over the fronto-parietal network might modify altered brain oscillations in patients who lack an optimized WM network, if after-effects would be prolonged with various stimulation sessions.

Overall, the results of this dissertation indicate that the anti-phase tACS-application interfered with ongoing theta coherence. Whereas there was no increase in WM performance on the group level for synchronization, the influence of tACS on behavior could be demonstrated by a deterioration in the WM task performance for desynchronization of the fronto-parietal network. Although the overall group performance for Condition C (synchronization) was not significantly altered to the sham group, individual analysis revealed differences in individual accuracy rates of the participants in the stimulation group. In previous studies similar findings were observed: anti-phase synchronization over the fronto-parietal network downgraded the WM performance (Polanía et al. 2012; Violante et al. 2017). However, in disagreement with our results, they claimed an improvement of the WM performance on account of fronto-parietal synchronization.

\subsection{Limitations of the Study}

In our study we were not able to replicate earlier findings related to tACS-synchronization over the fronto-parietal network (Polanía et al. 2012). At a first glance, it was surprising that the sham group scores (condition B) did not significantly differ from those of the synchronization group (condition A). There are several possible explanations for this result. First, a conceivable reason might be the fact that our targeted population included healthy and young subjects, who already have an individually optimized and balanced WM network. Under sham conditions, the subjects showed high accuracy rates and this has been suggested to influence the effect size of tACS-application (Kirov et al. 2009). Therefore, a clear limitation of this study is that only healthy university students were enrolled in the study. The impact of theta synchronization may show different results in participants who suffer from an impaired WM. Therefore, 
these outcomes might not be transferable to the wider population (e.g. elderly people and individuals with fewer years of education).

Second, the improvement due to synchronization of the PPC and dIPFC might have been cancelled out because of the simultaneous desynchronization of both hemispheres (see Figure 7). According to Nissim (2017), the optimal WM network shows brain activity lateralized to the right dIPFC (Nissim et al. 2017).

Third, the choice of a frequency in the upper theta range $(6 \mathrm{~Hz})$ could be accountable for insignificant effects of synchronization to directly improve the WM. We may speculate that the frequency of $6 \mathrm{~Hz}$ was too high and did not ideally match the individual theta range of the subjects (Vossen et al. 2015; Vosskuhl et al. 2016). We may only speculate if a slower theta frequency might have significant impacts on good performers. Instead, this might have had an impact on the effect size of the desynchronizing stimulation condition.

\subsection{Future Perspective}

The questions raised by this study are whether the synchronization condition would enhance the WM memory of an unbalanced fronto-parietal network and whether a slower theta frequency stimulation would alter the WM performance significantly. To resolve our open questions, further investigation and experimentation into in-phase tACS is recommended. We only observed online effects for the anti-phase tACS-application in the anterior-posterior direction. Future projects will have to examine whether a longer time period of in-phase and anti-phase tACS might show offline effects as well. Moreover, further studies will have to investigate, if the synchronization set up (condition C) could help participants with a deteriorated WM to reach an average performance on WM tasks during stimulation.

It would be interesting to assess the effects of tACS-application in another frequency and if this would influence the synchronizing stimulation condition. An argument in favor of a slower frequency in future studies is given by studies with tACS in the alpha frequency. If the stimulation matches the intrinsic brain oscillatory peaks, it would result in greater power and amplitude enhancement (Neuling et al. 2013; Vossen et al. 2015; Hanslmayr et al. 2016; Santarnecchi et al. 2016). We can reason that an individualized tACS-theta-frequency might show greater effects on the WM performance by synchronizing the fronto-parietal network. Taken together, this study should be repeated using a slower theta frequency in the range of 4$5 \mathrm{~Hz}$ and over the two hemispheres separately. After electrophysiological analysis of the individual theta frequency, tACS could also be matched to the intrinsic brain oscillations. 


\subsection{Clinical Relevance}

Psychiatric patients have longstanding been treated with pharmaceuticals, which complementarily intended to improve the accuracy of the WM in patients. Data from clinical and neuroanatomical investigations characterize psychiatric disorders by differences in structural gray and white matter, genetic expression and transmission between neurons (Voytek and Knight 2015). Moreover, Menon (2011) described dysfunctions of brain connectivity to result from deficits in participation or lacking involvement of relevant brain networks (Menon 2011). In psychopathologies such as schizophrenia and autism, an impaired communication between brain networks has been reported and that the collaboration of networks is crucial for cognitive as well as emotional regulation processes. The importance of synchronization of cortical networks is demonstrated by neurological and psychiatric disorders that are lacking oscillatory neuronal synchronization between networks, primarily schizophrenia and depression (Hipp et al. 2011). The growing understanding of the importance of connectivity and oscillatory activity in psychiatric and psychological diseases led to the new diagnostic term oscillopathy, which describes the abnormality or rather pathology of neuronal oscillations. A new approach targets the neuronal oscillations in patients suffering from psychological diseases.

Given the fact that certain pathologies have an increase or decrease in oscillatory activity in common, the clinical importance of our baseline study is underlined. In the present study, potential applications include further research and medical care of patients with a reduced WM capacity, including ADHD, schizophrenia, and depression (Martinussen et al. 2005; Rotzer et al. 2009; Szucs et al. 2013). Voytek and Knight hypothesized that abnormalities in cognition and behavior, as well as treatments, significantly modify dynamic network communications (Voytek and Knight 2015), and it has been reported that oscillatory coherence between regions is reduced in patients. According to Voytek and Knight, the balance of coupling is important (Voytek and Knight 2015). An increase in PAC would lead to increased coherence between the coupled frequencies and vice versa. Moreover, pathologically strong PAC might result from the feedback between oscillatory LFP and its influence on the neuronal population. Diseases associated with pathologic overcoupling such as depression could result from immoderately strengthened connections of networks, which associate negative emotions and life events. On the other hand, undercoupling of networks has been reported for diseases such as schizophrenia and autism. An example for undercoupling has been demonstrated in autism patients, where reductions in PAC between alpha and gamma frequencies have been reported. The pathologicnoise hypothesis claims that spikes preferentially occur during non-excitatory phases of lowfrequency oscillations, which would enlighten the theory of undercoupling in brain networks 
(Voytek and Knight 2015). The coherence between brain networks has been targeted by modulating the neocortex with low intensity transcranial brain stimulation (TBS), comprising subthreshold transcranial electric stimulation (TES) and supra-threshold transcranial magnetic stimulation (TMS). The application of low intensity TES has been associated with promising therapeutic effects on neurological and psychiatric illnesses (Lefaucheur et al. 2017). In fact, it has been suggested that tACS of weak current could be used for clinical purposes as an alternative solution for diseases correlated with modified oscillatory brain activity (Antal et al. 2008). The results of this study indicate that anti-phase tACS intervenes in the WM network. A potential development might be the application of non-invasive brain stimulation for treatment-resistant pathological brain activity in patients suffering from ADHD, Autism, depression or schizophrenia. We may speculate that tACS applied in the individual EEG frequency over the fronto-parietal network would modify altered brain oscillations in patients who lack of an optimized WM network, if after-effects would be prolonged with various stimulation sessions.

\subsection{Conclusion}

In the present work, we investigated the feasibility of bi-hemispheric manipulation of the human WM by applying tACS over the fronto-parietal network in the anterior-posterior direction. The results revealed that over-synchronization of the fronto-parietal network did not have significant group level effects, but on the individual level, volunteers with low baseline performance tended to improve due to the stimulation. At the same time, desynchronization of theta phase relations in the fronto-parietal network led to an impairment in the WM performance and decelerated the reaction time. Overall, we can conclude that the fronto-parietal WM network depends on an optimally balanced theta coherence, and this balance can be changed by multielectrode tACS. 


\section{Summary}

The WM is a key mechanism that ensures complex behavior in humans. It requires the bilateral activation of the fronto-parietal brain network and has been subdivided on the phonological loop, which processes numbers, semantics and auditory-verbal information, with lateralized activation towards the left hemisphere, and the visuo-spatial sketchpad, which mainly operates in the right hemisphere (Baddeley and Hitch 1974; Sauseng et al. 2005; Müller and Knight 2006; Baddeley 2012; Eriksson et al. 2015). This neural system maintains constant long-range communications within itself and with other cognitive systems through the neuronal oscillations.

This work aimed to show a causal relationship between visuospatial WM functions and the mechanism of fronto-parietal oscillatory connectivity - theta phase coherence. To reach our aim, two experiments were conducted. Our first hypothesis was that the WM network operates by phase connectivity. The results of the EEG experiment demonstrated significant theta coherence during the WM events. Our second hypothesis was that it would be feasible to interfere with the WM through bi-hemispheric tACS and thus change phase connectivity. To show causal effects of theta coherence, behavioral changes in subjects under fronto-parietal theta-tACS bilaterally, were analyzed. We found that desynchronization of theta phase relations in the frontoparietal network led to an impairment in the WM performance. Synchronization of the frontoparietal network had no significant effect on the group level, but individual analysis revealed a tendency for improvement of the WM for subjects with low baseline performance.

These results show that optimally balanced theta coherence in the fronto-parietal network is a prerequisite for the ideal WM functions in humans. 


\section{$6 \quad$ Attachments}

\subsection{Consent Form}

\section{UNIVERSITÄTSMEDIZIN $=\mathbf{M G}$
GÖTTINGEN $=\mathbf{O}$}

\section{Klinik für klinische Neurophysiologie}

Projektleiter: Prof. Dr. med. Walter Paulus

$$
\begin{aligned}
& \text { Ansprechpartner: } \\
& \text { Prof. Dr. med. Walter Paulus } \\
& \text { Leiter der Abteilung Klinische } \\
& \text { Neurophysiologie } \\
& \text { Medizinische Fakultät, } \\
& \text { Universität Göttingen } \\
& \text { Robert-Koch-Str. 40, } 37070 \\
& \text { Göttingen } \\
& \text { Tel.: } 0551 / 3966650 \text {, Telefax: } \\
& \text { 0551/398126 }
\end{aligned}
$$

\section{Einverständniserklärung zur Untersuchung:}

\section{Proving the concept of theta-gamma-ripple coupling in human neocortex}

Ich,

von einem Mitarbeiter der Abteilung Klinische Neurophysiologie vollständig über Wesen, Bedeutung und Tragweite der Magnetresonanz-Untersuchung sowie der transkraniellen Wechselstromstimulation und EEG aufgeklärt. Ich habe den Aufklärungstext gelesen und verstanden. Ich hatte die Möglichkeit, Fragen zu stellen. Ich habe ggfs. die Antworten verstanden und akzeptiere sie. Ein Mitarbeiter der Abteilung Klinische Neurophysiologie hat mich über die mit der Teilnahme an der Untersuchung verbundenen Risiken und den möglichen Nutzen informiert.

Ich hatte ausreichend Zeit, mich zur Teilnahme an dieser Untersuchung zu entscheiden und weiß, dass die Teilnahme freiwillig ist. Ich weiß, dass ich jederzeit und ohne Angaben von Gründen diese Zustimmung widerrufen kann, ohne dass sich dieser Entschluss nachteilig auf eventuell spätere ärztliche Behandlungen auswirken wird.

Mir ist bekannt, dass meine persönlichen Daten in verschlüsselter Form gespeichert werden. Mir ist bekannt, dass mein Name, mein Geburtsdatum, mein Gewicht, mein Geschlecht, meine Telefonnummer und meine Adresse in einer Kartei der Klinik für Klinische Neurophysiologie der Georg-August-Universität Göttingen gespeichert werden. Die Messdaten werden getrennt hiervon aufbewahrt. Ihre Verwendung erfolgt in namentlich nicht kenntlicher Form.

Obwohl die durchgeführte Untersuchung keine diagnostische Untersuchung ist, bestehtdie Möglichkeit, dass pathologische Befunde entdeckt werden (Zufallsfund). Sie haben die Wahlmöglichkeit, ob Sie in einem solchen Fall über den Zufallsfund informiert werden möchten oder nicht. Bitte kreuzen Sie entsprechend an: 


\section{UNIVERSITÄTSMEDIZIN GÖTTINGEN

Bilddaten, die auf einer eventuell ausgehändigten CD gespeichert sind, dürfen nicht für diagnostische Zwecke genutzt werden!

Die personenbezogenen Daten werden für 10 Jahre aufbewahrt. Mir ist bekannt, dass ich Auskunft über de gespeicherten Daten erhalten kann, und dass ich mein Einverständnis zur Speichenung der personenbezogenen Daten jederzeit widerrufen kann. Im Falle des Widernufs werden alle gespeicherten personenbezogenen Daten gelöscht.

Auf Wunsch erhalte ich eine Kopie des Informationsblattes und dieser Einwilligungserklärung. Ich erkläre hiermit meine freiwillige Teilnahme an dieser Untersuchung.

Ort/Datum:

Unterschrift (Testperson):

Ort/Datum:

Unterschrift (Wissenschaftlichen Mittarbeiter/in): 


\subsection{Participant Information}

\begin{tabular}{|c|c|}
\hline $\begin{array}{c}\text { UNIVERSITÄTSMEDIZIN } \\
\text { GÖTTINGEN }\end{array}$ & \\
\hline $\begin{array}{c}\text { Zentrum Neur ologische M ediż, Klinik für Klinische Neur ophysiologie } \\
\text { Robert-Koch-Str. 40, 37075 Göttingen } \\
\text { Direktor: Prof. Dr. med. Walter Paulus } \\
\text { Tel.: 0551/3966650 }\end{array}$ & UM G-NEUROPHYS \\
\hline \multicolumn{2}{|c|}{ Fragebogen } \\
\hline
\end{tabular}

\section{Probanden ID:}

\section{Teilnehmerinformationen}

1. Alter:

2. Geschlecht:

3. Geburtsjahr (JJJJ):

4. Wie viele Jahre haben Sie mit Schule, Ausbildung und Studium verbracht? (in Jahren):

5. Linkshänder oder Rechtshänder?

6. Haben Sie bereits in einem anderen Experiment mit elektrischer Stimulation teilgenommen? (a/ Nein)

Falls ja, bitte geben Sie an, an welchen Experimenten Sie teilgenommen haben!
I. TMS (Transkranielle Magnetstimulation ( $\underline{\mathrm{Ja}} / \underline{\mathrm{Nein}})$
II. TES (Transkranielle Stromstimulation) (미 / Nein)

7. Trinken Sie regelmäßig Kaffee? (므 / $\underline{\text { Nein })}$

Falls ja, bitte geben Sie an, wie oft trinken Sie Kaffee pro Tag:

8. Trinken Sie regelmäßig Alkohol? ( $\underline{\mathrm{Ja}} / \underline{\text { Nein }})$

Falls ja, bitte geben Sie an, wie oft trinken Sie Alkohol pro Woche:

9. Rauchen Sie? (a / Nein)

Falls ja, bitte geben Sie an, wie oft rauchen pro Tag:

10. Haben Sie Kopfschmerzen? (aㅡ / Nein)

Falls ja, wie oft haben Sie Kopfschmerzen? (Bitte Kreis)
I. Jeden Tag
II. Mehrmals pro Woche
III. Einmal pro Woche
IV. Mehrmals pro Monat
V. Weniger als einmal pro Monat

Falls ja, bitte geben Sie an, wie intensiv Ihre Kopfschmerzen sind!

$(1=$ gering $-10=$ nicht aushaltbar $)$ : 


\subsection{Health Questionnaire}

\begin{tabular}{r|c}
\hline $\begin{array}{c}\text { UNIVERSITÄTSMEDIZIN } \\
\text { GÖTTINGEN }=\end{array}$ & \\
\hline $\begin{array}{c}\text { Zentrum Neurologische Medizin, Klinik für Klinische Neurophysiologie } \\
\text { Robert-Koch-Str. 40, 37075 Göttingen } \\
\text { Direktor: Prof. Dr. med. Walter Paulus } \\
\text { Tel.: 0551/3966650 }\end{array}$ & UMG-NEUROPHYS \\
\hline
\end{tabular}

Fragebogen zur transkraniellen Gleichstrom / Wechselstrom / Magnetstimulation

Lesen Sie sich zu Ihrer eigenen Sicher heit diesen Fragebogen gründlich durch und beantworten Sie gewissenhaft alleFragen. Wenn Sie sich nicht sicher sind oder eine Fragenicht verstehen, wenden Sie sich bitte an einen unser Mitar beiter. Unter schreiben Sie anschließend den Fragebogen und lasen Sie sich von einem Mitar beiter einweisn, bevor Siean der Studie teilnehmen können.

Anweisung. Bitte vollständig und gut lesbar ausfüllen. Bitte kreuzen Sie immer die Antwort an.

\begin{tabular}{|c|c|c|}
\hline 1 & Sind Sie jünger als $18 ?$ & $\square$ JA $\square$ NEIN \\
\hline 2 & $\begin{array}{l}\text { Ist bei Ihnen ein Anfallsleiden (Epilepsie, inkl. kindlicher Absencen) } \\
\text { bekannt? }\end{array}$ & $\square$ JA $\square$ NEIN \\
\hline 3 & $\begin{array}{l}\text { Ist in Ihrer unmittelbaren Familie (Eltern, Geschwister) eine Epilepsie } \\
\text { bekannt? }\end{array}$ & $\square$ JA $\square$ NEIN \\
\hline 4 & Haben Sie schon Ihr Bewusstsein verloren? Wenn ja, wann? & $\square$ JA $\square$ NEIN \\
\hline 5 & $\begin{array}{l}\text { Sind bei Ihnen andere neurologische oder psychiatrische Erkrankungen } \\
\text { (inklusive -Alkohol-, Medikamenten- und Drogenabhängigkeit oder - } \\
\text { mißbrauch) bekannt? Wennja, welche? }\end{array}$ & $\square$ JA $\square$ NEIN \\
\hline 6 & $\begin{array}{l}\text { Leiden Sie unter einer schweren Erkrankung der Atemwege, des Herz- } \\
\text { Kreislaufsystems oder des Bewegungssystems (z.B. Asthma, Diabetes, } \\
\text { Herzschwäche, Herzrhythmusstörungen, Lähmungen)? Wenn ja, welche? }\end{array}$ & $\square$ JA $\square$ NEIN \\
\hline 7 & $\begin{array}{l}\text { Wurde bei Ihnen je zu diagnostischen Zwecken ein EEG oder MRT } \\
\text { gemacht? }\end{array}$ & $\square$ JA $\square$ NEIN \\
\hline 8 & Hatten Sie je behandlungsbedürftige Kopfverletzungen? & $\square$ JA $\square$ NEIN \\
\hline 9 & Leiden Sie regelmäßig an Kopfschmerzen? & $\square$ JA $\square$ NEIN \\
\hline
\end{tabular}




\begin{tabular}{|c|l|l|}
\hline 11 & $\begin{array}{l}\text { Haben Sie -Metallimplantate im Kopf- und Wirbelsäulebereich (in den } \\
\text { Kopfbereich eingesetztes Metal, z.B. Clips nach Operation eines } \\
\text { intrazerebralen Aneurysmas (Gefäßaussackung im Bereich der } \\
\text { Gehirngefäße), Implantation eine künstlichen Hörschnecke) }\end{array}$ & $\square$ JA $\square$ NEIN \\
\hline 12 & $\begin{array}{l}\text { Sind Sie Träger eines Herzschrittmachers, Defibrillators, Hörgeräts, } \\
\text { Medikamentenpumpe (Insulin?), Neurostimulators, Implantat mit } \\
\text { Magnetventil (z.B. künstlicher Darmausgang)? Wenn ja, welche? }\end{array}$ & $\square$ JA $\square$ NEIN \\
\hline 13 & \begin{tabular}{l} 
Leiden Sie an Schlafstörungen? \\
\hline 14
\end{tabular} Haben Sie Herzrhythmusstörungen? & $\square$ JA $\square$ NEIN \\
\hline 15 & Wurden Sie innerhalb der letzten zwei Monate operiert? Wenn ja, woran? \\
\hline 16 & $\begin{array}{l}\text { Haben Sie in den letzten 5 Tagen an einer MRT-., tDCS-, tACS- oder TMS- } \\
\text { Untersuchung teilgenommen? }\end{array}$ & $\square$ JA $\square$ NEIN \\
\hline 17 & $\begin{array}{l}\text { Nehmen Sie zur Zeit gleichzeitig an einem anderem Experiment teil? } \\
\text { Wenn ja, woran? }\end{array}$ & $\square$ JEIN \\
\hline
\end{tabular}

\section{Nur von Frauen auszufüllen:}

\begin{tabular}{|l|l|l|}
\hline 18 & Besteht die Möglichkeit, dass Sie schwanger sind? & $\square$ JA $\square$ NEIN \\
\hline 19 & Stillen Sie im Moment? & $\square$ JA $\square$ NEIN \\
\hline
\end{tabular}

Ich habe die Fragen gelesen und verstanden. Ich erkläre darüber hinaus, dass ich alle Angaben zur Krankengeschichte wahrheitsgemäß gemacht habe.

(Datum, Ort)

(Name, Vorname)

(Unterschrift) 
Probanden ID:

RR:

Puls:

Körpergröße:

Gewicht:

Medikation (ggf.):

Gang:

Okulomotorik:

Auffälligkeiten (Haut, Sprache, usw):

— Vorerkrankungen -

Epilepsie:

Migräne:

Metallimplantate:

Herzerkrankungen:

Operationen:

Chronische und genetische Krankheiten:

(Datum, Ort)

(Name, Vorname)

(Unterschrift) 


\subsection{After-Session Questionnaire}

\begin{tabular}{|c|c}
\hline $\begin{array}{c}\text { UNIVERSITÄTSMEDIZIN } \\
\text { GÖTTINGEN }\end{array}$ & \\
\hline $\begin{array}{c}\text { Zentr um Neur ologische M edizn, Klinik für Klinische Neur ophysiologie } \\
\text { Rober t-Koch-Str. 40, 37075 Göttingen } \\
\text { Direktor: Prof. Dr. med. Walter Paulus } \\
\text { Tel.: 0551/3966650 }\end{array}$ & UM G-NEUROPHYS \\
\hline
\end{tabular}

\section{Study code:}

Participant ID:

Session:

Date and Time:

Questionnaire

1. Wie viele Stunden haben Sie in der letzten Nacht geschlafen?

2. Bitte schätzen Sie, wie gut Sie geschlafen haben auf einer Skala von 1 bis 5 ! (1: sehr schlecht -5 : sehr gut):

3. Wie viele Stunden brauchten Sie zum Einschlafen?

4. Wie oft sind Sie während der letzten Nacht aufgewacht?

5. Haben Sie heute Kaffee getrunken? (므 / Nein)

Falls ja, geben Sie bitte an, wann Sie letzten Kaffee getrunken haben?

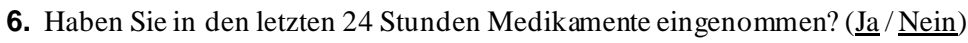

Falls ja, bitte geben Sie die Namen der Medikamente an, und wann Sie diese eingenommen haben.

7. Haben Sie in den letzten 48 Stunden Alkohol getrunken?(므/ ein)

Falls ja, geben Sie bitte an, wie viel Alkohol Sie getrunken haben. (Bitte Kreis)

- wenig

- mäßig 
8. Denken Sie, dass die Stimulation Ihre Leistung verändert hat?

JA $\square$

NEIN

- Falls ja:

Verbessert

Verschlechtert $\square$

- Falls ja, was denken Sie, wie stark war dieser Effekt in Prozent?

(z.B. durch die Stimulation wird sich meine Leistung um $50 \%$ verbessern/ verschlechtern)

9. Haben Sie während der Stimulation Lichtblitze wahrgenommen?

JA

NEIN

- Falls ja, bitte geben Sie an, wann Sie Lichtblitze gefühlt haben!

am Anfang

in der Mitte

am Ende

- Falls ja, wie stark haben Sie die Lichtblitze gespürt?

$1=$ gering $-10=$ sehr stark:

- Falls ja, verspüren Sie im Moment Lichtblitze?

JA

NEIN

10. Hat Ihre Kopfhaut während der Stimulation unter den Elektroden gejuckt?

JA

NEIN

- Falls ja, bitte geben Sie an, wann Sie das Jucken gefühlt haben!

am Anfang

in der Mitte

am Ende

- Falls ja,wie stark haben Sie das Jucken gespürt?

$1=$ gering $-10=$ sehr stark:

- Falls ja, verspüren Sie das Jucken im Moment?

JA

$\square$

NEIN 
11. Hat Ihre Kopfhaut während der Stimulation unter den Elektroden gebrannt?

JA $\square$

NEIN

- Falls ja, bitte geben Sie an, wann Sie das Brennen gefühlt haben!

am Anfang

$\square$

in der Mitte

$\square$

am Ende

- Falls ja, wie stark haben Sie das Brennen gespürt?

$1=$ gering $-10=$ sehr stark:

- Falls ja, verspüren Sie das Brennen im Moment?

JA

NEIN

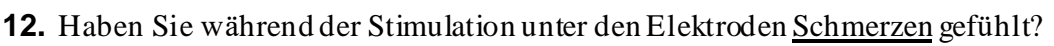

JA

NEIN

- Falls ja, bitte geben Sie an, wann Sie den Schmerz gefühlt haben!

am Anfang

in der Mitte

am Ende

- Falls ja, wie stark haben Sie den Schmerz gespürt?

$1=$ gering $-10=$ sehr stark:

- Falls ja, verspüren Sie den Schmerz im Moment?

JA

NEIN

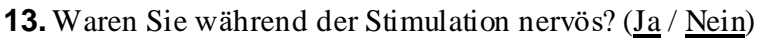

Falls ja, wie nervös waren Sie? ( 1 = gering- $10=$ sehr nervös):

14. Haben Sie während der Stimulation Kopfschmerzen wahrgenommen? (므 / $\underline{\text { Nein }})$

Falls ja, wie stark waren Ihre Kopfschmerzen? $(1=$ gering $-10=$ sehr stark $)$ :

15. Wie fühlen Sie sich im Moment? $(1=$ sehr müde $-10=$ vollkommen wach $)$ :

16. Wie schwierig fanden Sie die Aufgabe? $(1=\operatorname{sehr}$ einfach $-10=$ sehr schwierig): 


\section{$7 \quad$ References}

Aggleton JP, Pralus A, Nelson AJD, Hornberger M (2016): Thalamic pathology and memory loss in early Alzheimer's disease: moving the focus from the medial temporal lobe to Papez circuit. Brain 139, 1877-1890

Alekseichuk I, Pabel SC, Antal A, Paulus W (2017): Intrahemispheric theta rhythm desynchronization impairs working memory. Restor Neurol Neurosci $\underline{35}$, 147-158

Allen RJ, Havelka J, Falcon T, Evans S, Darling S (2015): Modality specificity and integration in working memory: Insights from visuospatial bootstrapping. J Exp Psychol Learn Mem $\operatorname{Cogn} \underline{41}, 820-830$

Anastassiou C a, Perin R, Markram H, Koch C (2011): Ephaptic coupling of cortical neurons. Nat Neurosci 14, 217-223

Anschutz L, Camp C, Markley R, Kramer J (1987): Remembering mnemonics: A three-year follow-up on the effects of mnemonics training in elderly adults. Exp Aging Res $\underline{13}, 141-$ 143

Antal A, Paulus W (2013): Transcranial alternating current stimulation (tACS). Front Hum Neurosci 7, 317

Antal A, Herrmann CS (2016): Transcranial Alternating Current and Random Noise Stimulation: Possible Mechanisms. Neural Plast 2016, 3616807

Antal A, Boros K, Poreisz C, Chaieb L, Terney D, Paulus W (2008): Comparatively weak aftereffects of transcranial alternating current stimulation (tACS) on cortical excitability in humans. Brain Stimul 1, 97-105

Antal A, Alekseichuk I, Bikson M, Brockmöller J, Brunoni AR, Chen R, Cohen LG, Dowthwaite G, Ellrich J, Flöel A, et al. (2017): Low intensity transcranial electric stimulation: Safety, ethical, legal regulatory and application guidelines. Clin Neurophysiol $\underline{128}, 1774-1809$

Atkinson RC, Shiffrin RM (1968): Human memory: A proposed system and its control processes. In: Spence KW, Spence JT (Hrsg.): The psychology of learning and motivation: Advances in research and theory. Band 2. Academic Press, New York 1968, 89-195

Baddeley A (2000): The episodic buffer: A new component of working memory? Trends Cogn Sci $\underline{4}, 417-423$

Baddeley A (2001): The concept of episodic memory. Philos Trans R Soc Lond B Biol Sci $\underline{36}$, $1345-1350$

Baddeley A (2012): Working Memory: Theories, Models, and Controversies. Annu Rev Psychol $\underline{63}, 1-29$

Baddeley A, Hitch G (1974): Working memory. Psychol Learn Motiv $\underline{8}$, 47-89

Bannerman DM, Sprengel R, Sanderson DJ, McHugh SB, Rawlins JNP, Monyer H, Seeburg 
PH (2014): Hippocampal synaptic plasticity, spatial memory and anxiety. Nat Rev Neurosci 15 , 181-92

Bartsch T, Wulff P (2015): The hippocampus in aging and disease: From plasticity to vulnerability. Neuroscience $\underline{309}, 1-16$

Battleday RM, Muller T, Clayton MS, Kadosh RC (2014): Mapping the mechanisms of transcranial alternating current stimulation: A pathway from network effects to cognition. Front Psychiatry $\underline{5}, 1-5$

Bavelier D, Newman AJ, Mukherjee M, Hauser P, Kemeny S, Braun A, Boutla M (2008): Encoding, rehearsal, and recall in signers and speakers: Shared network but differential engagement. Cereb Cortex 18, 2263-2274

Baxter MG (2009): Involvement of Medial Temporal Lobe Structures in Memory and Perception. Neuron $\underline{61}, 667-677$

Benton AL (1968): Differential Behavioral Effects in Frontal Lobe Disease. Neuropsychologia $\underline{6}, 53-60$

Bereket A, Turan S, Karaman MG, Haklar G, Ozbay F, Yazgan MY (2005): Height, weight, IGF-I, IGFBP-3 and thyroid functions in prepubertal children with attention deficit hyperactivity disorder: Effect of methylphenidate treatment. Horm Res $\underline{63}$, 159-164

Berlucchi G, Buchtel HA (2009): Neuronal plasticity: Historical roots and evolution of meaning. Exp Brain Res 192, 307-319

Bonnet-Brilhault F (2017): L'autisme : un trouble neuro-développemental précoce. Arch Pediatr 24, 384-390

Bosch M, Castro J, Saneyoshi T, Matsuno H, Sur M, Hayashi Y (2014): Structural and molecular remodeling of dendritic spine substructures during long-term potentiation. Neuron $\underline{82}$, $444-459$

Burle B, Bonnet M (2000): High-speed memory scanning: A behavioral argument for a serial oscillatory model. Cogn Brain Res $\underline{9}, 327-337$

Buschkuehl M, Hernandez-Garcia L, Jaeggi SM, Bernard J a, Jonides J (2014): Neural effects of short-term training on working memory. Cogn Affect Behav Neurosci 14, 147-160

Buzsáki G (2010): Neural Syntax: Cell Assemblies, Synapsembles, and Readers. Neuron $\underline{68}$, 362-385

Buzsáki G, Anastassiou C a, Koch C (2012): The origin of extracellular fields and currents-EEG, ECoG, LFP and spikes. Nat Rev Neurosci $\underline{13}$, 407-420

Caeyenberghs K, Metzler-Baddeley C, Foley S, Jones DK (2016): Dynamics of the Human Structural Connectome Underlying Working Memory Training. J Neurosci $\underline{\text { 36, 4056-4066 }}$ 
Cameron HA, Glover LR (2015): Adult Neurogenesis : Beyond Learning and Memory. Annu Rev Psychol 66, 53-81

Canolty RT, Edwards E, Dalal SS, Soltani M, Nagarajan SS, Berger MS, Barbaro NM, Knight RT, Kirsch HE, Berger MS, et al. (2009): High Gamma Power is Phase-Locked to Theta Oscillations in Human Neocortex. Science 313, 1626-1628

Chaieb L, Antal A, Pisoni A, Saiote C, Opitz A, Ambrus GG, Focke N, Paulus W (2014): Safety of $5 \mathrm{kHz}$ tACS. Brain Stimul 7, 92-96

Chaudhuri R, Fiete I (2016): Computational principles of memory. Nat Neurosci $\underline{19}$, 394-403

Christophel TB, Klink PC, Spitzer B, Roelfsema PR (2017): The Distributed Nature of Working Memory. Trends Cogn Sci 21, 111-124

Citri A, Malenka RC (2008): Synaptic Plasticity: Multiple Forms, Functions, and Mechanisms. Neuropsychopharmacology $\underline{33}, 18-41$

Cohen MX (2011): It's about Time. Front Hum Neurosci $\underline{5}, 2$

Cohen MX: Analyzing Neural Time Series Data: Theory and Practice. MIT, Cambridge, MA, 2014

Constantinidis C, Klingberg T (2016): The neuroscience of working memory capacity and training. Nat Rev Neurosci 17, 438-449

Cowan N (2001): The magical number 4 in short term memory. A reconsideration of storage capacity. Behav Brain Sci 24, 87-186

Cowan N (2010): The Magical Mystery Four: How Is Working Memory Capacity Limited, and Why? Curr Dir Psychol Sci 19, 51-57

Cowan N (2015): George Miller's magical number of immediate memory in retrospect: Observations on the faltering progression of science. Psychol Rev 122, 536-541

Cowan N (2017): Mental Objects in Working Memory: Development of Basic Capacity or of Cognitive Completion? Adv Child Dev Behav 52, 81-104

Cowan N, Elliott EM, Saults SJ, Morey CC, Mattox S, Hismjatullina A, Conway AR (2005): On the capacity of attention: Its estimation and its role in working memory and cognitive aptitudes. Cogn Psychol 11, 42-100

Curti S, O’Brien J (2016): Characteristics and plasticity of electrical synaptic transmission. BMC Cell Biol 17, 13

D 'Esposito M, Postle BR (2015): The Cognitive Neuroscience of Working Memory. Annu Rev Psychol $\underline{66}, 115-42$

Darki F, Klingberg T (2015): The role of fronto-parietal and fronto-striatal networks in the development of working memory: A longitudinal study. Cereb Cortex $\underline{25}$, 1587-1595

Darling S, Havelka J (2010): Visuospatial bootstrapping: Evidence for binding of verbal and 
spatial information in working memory. QJ Exp Psychol (Hove) 63, 239-245

Devaraju P, Yu J, Eddins D, Mellado-Lagarde MM, Earls LR, Westmoreland JJ, Quarato G, Green DR, Zakharenko SS (2017): Haploinsufficiency of the 22q11.2 microdeletion gene Mrp140 disrupts short-term synaptic plasticity and working memory through dysregulation of mitochondrial calcium. Mol Psychiatry 22, 1313-1326

Dresler M, Shirer WR, Konrad BN, Müller NCJ, Wagner IC, Fernández G, Czisch M, Greicius MD (2017): Mnemonic Training Reshapes Brain Networks to Support Superior Memory. Neuron $\underline{\text { 93, }}$ 1227-1235

Duff SJ, Hampson E (2001): A Sex Difference on a Novel Spatial Working Memory Task in Humans. Brain Cogn 47, 470-493

Eriksson J, Vogel EK, Lansner A, Bergström F, Nyberg L (2015): Neurocognitive Architecture of Working Memory. Neuron $\underline{88}, 33-46$

Fakhri M, Sikaroodi H, Maleki F, Ali Oghabian M, Ghanaati H (2012): Age-related frontal hyperactivation observed across different working memory tasks: An fMRI study. Behav Neurol 25, 351-361

Fell J, Axmacher N (2011): The role of phase synchronization in memory processes. Nat Rev Neurosci 12 , 105-118

Fellner M-C, Volberg G, Wimber M, Goldhacker M, Greenlee MW, Hanslmayr S (2016): Spatial mnemonic encoding: Theta power decreases co-occur with medial temporal lobe BOLD increases during the usage of the Method of Loci. eNeuro $\underline{3}$, pii: ENEURO.0184-16.2016

Fries P (2005): A mechanism for cognitive dynamics: Neuronal communication through neuronal coherence. Trends Cogn Sci $\underline{9}, 474-480$

Fröhlich F, McCormick DA (2010): Endogenous electric fields may guide neocortical network activity. Neuron $\underline{67}, 129-143$

Fröhlich F, Sellers KK, Cordle AL (2015): Targeting the neurophysiology of cognitive systems with transcranial alternating current stimulation. Expert Rev Neurother 15, 145-167

Getzmann S, Gajewski PD, Falkenstein M (2013): Does age increase auditory distraction? Electrophysiological correlates of high and low performance in seniors. Neurobiol Aging $\underline{34}, 1952-1962$

Gignac GE (2015): The magical numbers 7 and 4 are resistant to the Flynn effect: No evidence for increases in forward or backward recall across 85 years of data. Intelligence $\underline{48}, 85-95$

Griesmayr B, Gruber WR, Klimesch W, Sauseng P (2010): Human frontal midline theta and its synchronization to gamma during a verbal delayed match to sample task. Neurobiol Learn Mem 르, 208-215

Griesmayr B, Berger B, Stelzig-Schoeler R, Aichhorn W, Bergmann J, Sauseng P (2014): EEG 
theta phase coupling during executive control of visual working memory investigated in individuals with schizophrenia and in healthy controls. Cogn Affect Behav Neurosci $\underline{14}$, 1340-1355

Grieve SM, Clark CR, Williams LM, Peduto AJ, Gordon E (2005): Preservation of Limbic and Paralimbic Structures in Aging. Hum Brain Mapp 25, 391-401

Groenman AP, Schweren LJ, Dietrich A, Hoekstra PJ (2017): An update on the safety of psychostimulants for the treatment of attention-deficit/hyperactivity disorder: Expert Opin Drug Saf 16, 455-464

Hanslmayr S, Staresina BP, Bowman H (2016): Oscillations and Episodic Memory: Addressing the Synchronization/Desynchronization Conundrum. Trends Neurosci $\underline{39}, 16-25$

Helfrich RF, Schneider TR, Rach S, Trautmann-Lengsfeld SA, Engel AK, Herrmann CS (2014a): Entrainment of brain oscillations by transcranial alternating current stimulation. Curr Biol 24, 333-339

Helfrich RF, Knepper H, Nolte G, Strüber D, Rach S, Herrmann CS, Schneider TR, Engel AK (2014b): Selective Modulation of Interhemispheric Functional Connectivity by HD-tACS Shapes Perception. PLoS Biol 12, e1002031

Henseler I, Falkai P, Gruber O (2010): Disturbed functional connectivity within brain networks subserving domain-specific subcomponents of working memory in schizophrenia: Relation to performance and clinical symptoms. J Psychiatr Res 44, 364-372

Hermans EJ, Kanen JW, Tambini A, Fernández G, Davachi L, Phelps EA (2016): Persistence of Amygdala-Hippocampal Connectivity and Multi-Voxel Correlation Structures During Awake Rest After Fear Learning Predicts Long-Term Expression of Fear. Cereb Cortex 27, 3028-3041

Herrmann CS, Rach S, Neuling T, Strüber D (2013): Transcranial alternating current stimulation: a review of the underlying mechanisms and modulation of cognitive processes. Front Hum Neurosci ㄱ, 279

Herrmann CS, Strüber D, Helfrich RF, Engel AK (2016): EEG oscillations: From correlation to causality. Int J Psychophysiol $\underline{103}, 12-21$

Hill AC, Laird AR, Robinson JL (2014): Gender differences in working memory networks: a BrainMap meta-analysis. Biol Psychol 102, 18-29

Hipp JF, Engel AK, Siegel M (2011): Oscillatory synchronization in large-scale cortical networks predicts perception. Neuron $\underline{69}, 387-396$

Holdefer RN, Sadleir R, Russell MJ (2006): Predicted current densities in the brain during transcranial electrical stimulation. Clin Neurophysiol 117, 1388-1397 
Hutcheon B, Yarom Y (2000): Resonance, oscillation and the intrinsic frequency preferences of neurons. Trends Neurosci 23 , 216-222

Intlekofer KA, Cotman CW (2013): Exercise counteracts declining hippocampal function in aging and Alzheimer' s disease. Neurobiol Dis $\underline{57}, 47-55$

Izhikevich EM, Desai NS, Walcott EC, Hoppensteadt FC (2003): Bursts as a unit of neural information: Selective communication via resonance. Trends Neurosci 26, 161-167

Jackson AF, Bolger DJ (2014): The neurophysiological bases of EEG and EEG measurement: A review for the rest of us. Psychophysiology $\underline{51}, 1061-1071$

Jaušovec N, Jaušovec K (2014): Increasing working memory capacity with theta transcranial alternating current stimulation (tACS). Biol Psychol $\underline{96}$, 42-47

Jaušovec N, Jaušovec K, Pahor A (2014): The influence of theta transcranial alternating current stimulation (tACS) on working memory storage and processing functions. Acta Psychol (Amst) $146,1-6$

Kaminski J, Brzezicka A, Wróbel A (2011): Short-term memory capacity (7士2) predicted by theta to gamma cycle length ratio. Neurobiol Learn Mem $\underline{95}, 19-23$

Schacter DL, Wagner AD: Learning and Memory. In: Kandel ER, Schwartz JH, Jessell TM, Siegelbaum SA, Hudspeth AJ (Hrsg.): Principles of Neural Science. 5.Aufl.; McGraw-Hill, o.O. $2013,1441-1460$

Kastellakis G, Cai DJ, Mednick SC, Silva AJ, Poirazi P (2015): Synaptic clustering within dendrites: An emerging theory of memory formation. Prog Neurobiol 126, 19-35

Kaufman SB (2007): Sex differences in mental rotation and spatial visualization ability: Can they be accounted for by differences in working memory capacity? Intelligence $\underline{35}, 211-$ 223

Kawasaki M, Kitajo K, Yamaguchi Y (2014): Fronto-parietal and fronto-temporal theta phase synchronization for visual and auditory-verbal working memory. Front Psychol $\underline{5}, 200$

Kesner RP, Rolls ET (2015): A computational theory of hippocampal function, and tests of the theory: New developments. Neurosci Biobehav Rev $\underline{48}$, 92-147

Khader PH, Jost K, Ranganath C, Rösler F (2010): Theta and alpha oscillations during workingmemory maintenance predict successful long-term memory encoding. Neurosci Lett $\underline{468}$, 339-343

Kikuchi M, Yoshimura Y, Hiraishi H, Munesue T, Hashimoto T, Tsubokawa T, Takahashi T, Suzuki M, Higashida H, Minabe Y (2015): Reduced long-range functional connectivity in young children with autism spectrum disorder. Soc Cogn Affect Neurosci 10, 248-254

Kim J-J, Kwon JS, Park HJ, Youn T, Kim MS, Lee DS, Lee MC (2003): Functional Disconnection Between the Prefrontal and Parietal Cortices During Working Memory 
Processing in Schizophrenia: A [ 15 O]H2O PET Study. Am J Psychiatry 160, 919-923

Kirov R, Weiss C, Siebner HR, Born J, Marshall L (2009): Slow oscillation electrical brain stimulation during waking promotes EEG theta activity and memory encoding. Proc Natl Acad Sci 106, 15460-15465

Klingberg T (2010): Training and plasticity of working memory. Trends Cogn Sci 14, 317-324

Lea G (1975): Chronometric analysis of the method of loci. J Exp Psychol Hum Percept Perform 1, 95-104

Lefaucheur J-P, Antal A, Ayache SS, Benninger DH, Brunelin J, Cogiamanian F, Cotelli M, De Ridder D, Ferrucci R, Langguth B, et al. (2017): Evidence-based guidelines on the therapeutic use of transcranial direct current stimulation (tDCS). Clin Neurophysiol $\underline{128}$, 56-92

Lemaitre H, Goldman AL, Sambataro F, Verchinski BA, Meyer-Lindenberg A, Weinberger DR, Mattay VS (2012): Normal age-related brain morphometric changes : nonuniformity across cortical thickness, surface area and gray matter volume ? Neurobiol Aging $\underline{33}$, 617.e1-9

Li X, Xiao Y-H, Zhao Q, Leung AWW, Cheung EFC, Chan RCK (2015): The neuroplastic effect of working memory training in healthy volunteers and patients with schizophrenia: Implications for cognitive rehabilitation. Neuropsychologia $\underline{75}, 149-162$

Lisman J (2010): Working memory: The importance of theta and gamma oscillations. Curr Biol 20, R490-R492

Loh E, Kumaran D, Koster R, Berron D, Dolan R, Duzel E (2016): Context-specific activation of hippocampus and SN/VTA by reward is related to enhanced long-term memory for embedded objects. Neurobiol Learn Mem 134, 65-77

Lopes da Silva F: EEG: Origin and Measurement. In: Mulert C, Lemieux L (Hrsg.): EEG-fMRI Physiological Basis, Technique, and Applications. Springer, o.O. 2010, 19-39

Lundqvist M, Herman P, Lansner A (2011): Theta and gamma power increases and alpha/beta power decreases with memory load in an attractor network model. J Cogn Neurosci $\underline{23}$, 3008-3020

Luria AR: The Mind of a Mnemonist: A Little Book About a Vast Memory. Basic Books, Inc., New York 1968

Ma WJ, Husain M, Bays PM (2014): Changing concepts of working memory. Nat Neurosci $\underline{17}$, $347-356$

MacDonald AW, Cohen JD, Stenger VA, Carter CS (2000): Dissociating the Role of the Dorsolateral Prefrontal and Anterior Cingulate Cortex in Cognitive Control. Science 288, 1835-1838 
Maris E, Fries P, van Ede F (2016): Diverse Phase Relations among Neuronal Rhythms and Their Potential Function. Trends Neurosci 39, 86-99

Martinussen R, Hayden J, Hogg-Johnson S, Tannock R (2005): A Meta-Analysis of Working Memory Impairments in Children With Attention-Deficit/Hyperactivity Disorder. J Am Acad Child Adolesc Psychiatry $\underline{44}, 377-384$

Meiron O, Lavidor M (2014): Prefrontal oscillatory stimulation modulates access to cognitive control references in retrospective metacognitive commentary. Clin Neurophysiol $\underline{125}$, $77-82$

Meiron O, Hermesh H, Katz N, Weizman A (2013): Executive attention deficits in schizophrenia: Putative mandatory and differential cognitive pathology domains in medicated schizophrenia patients. Psychiatry Res 209, 1-8

Menon V (2011): Large-scale brain networks and psychopathology: A unifying triple network model. Trends Cogn Sci $\underline{15}, 483-506$

Miller EK, Buschman TJ (2015): Working Memory Capacity: Limits on the Bandwidth of Cognition. Daedalus $\underline{144}, 112-122$

Miller G (1956): The magical number seven, plus or minus two: some limits on our capacity for processing information. Psychol Rev 101, 343-352

Miranda PC, Mekonnen A, Salvador R, Ruffini G (2013): The electric field in the cortex during transcranial current stimulation. Neuroimage $\underline{70}$, 48-58

Müller NG, Knight RT (2006): The functional neuroanatomy of working memory: Contributions of human brain lesion studies. Neuroscience 139, 51-58

Neuling T, Wagner S, Wolters CH, Zaehle T, Herrmann CS (2012): Finite-element model predicts current density distribution for clinical applications of tDCS and tACS. Front Psychiatry $\underline{3}, 83$

Neuling T, Rach S, Herrmann CS (2013): Orchestrating neuronal networks: sustained aftereffects of transcranial alternating current stimulation depend upon brain states. Front Hum Neurosci 7, 161

Nicoll RA (2017): Review A Brief History of Long-Term Potentiation. Neuron 93, 281-290

Nissim NR, O’Shea AM, Bryant V, Porges EC, Cohen R, Woods AJ (2017): Frontal Structural Neural Correlates of Working Memory Performance in Older Adults. Front Aging Neurosci $\underline{8}, 328$

Nyberg L, Lövden M, Riklund K, Lindenberger U, Bäckman L (2012): Memory aging and brain maintenance. Trends Cogn Sci 16, 292-305

Olesen PJ, Westerberg H, Klingberg T (2004): Increased prefrontal and parietal activity after training of working memory. Nat Neurosci $\underline{7}, 75-79$ 
Omerhodzic I, Avdakovic S, Nuhanovic A, Dizdarevic K (2010): Energy Distribution of EEG Signals : EEG Signal Wavelet-Neural Network Classifier. Neurocomputing 2, 210-215

Onton J, Delorme A, Makeig S (2005): Frontal midline EEG dynamics during working memory. Neuroimage 27, 341-356

Opitz A, Falchier A, Yan C, Yeagle E, Linn G (2016): Spatiotemporal structure of intracranial electric fields induced by transcranial electric stimulation in human and nonhuman primates. Sci Rep $\underline{6}, 31236$

Oren N, Ash EL, Tarrasch R, Hendler T, Giladi N, Shapira-Lichter I (2017): Neural patterns underlying the effect of negative distractors on working memory in older adults. Neurobiol

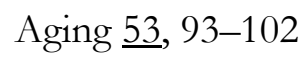

Palva JM, Monto S, Kulashekhar S, Palva S (2010): Neuronal synchrony reveals working memory networks and predicts individual memory capacity. Proc Natl Acad Sci U S A $\underline{107}$, $7580-7585$

Papez JW (1937): A proposed mechanism of emotion. J Neuropsychiatry Clin Neurosci , 103112

Pasternak T, Greenlee MW (2005): Working memory in primate sensory systems. Nat Rev Neurosci $\underline{6}, 97-107$

Petrides M (1994): Frontal lobes and behaviour. Curr Biol 4, 207-211

Polanía R, Nitsche MA, Korman C, Batsikadze G, Paulus W (2012): The importance of timing in segregated theta phase-coupling for cognitive performance. Curr Biol 22, 1314-1318

Proskovec AL, Heinrichs-Graham E, Wilson TW (2016): Aging modulates the oscillatory dynamics underlying successful working memory encoding and maintenance. Hum Brain Mapp 2361, 2348-2361

Purves D, Augustine GJ, Fitzpatrick D, Hall WC, LaMantia AS, McNamara JO, White LE: Neuroscience. 4. Aufl.; Sinauer Associates, Sunderland, MA, 2008

Qureshi A, Rizvi F, Syed A, Shahid A, Manzoor H (2014): The method of loci as a mnemonic device to facilitate learning in endocrinology leads to improvement in student performance as measured by assessments. Adv Physiol Educ 38, 140-144

Reato D, Rahman A, Bikson M, Parra LC (2013): Effects of weak transcranial alternating current stimulation on brain activity-a review of known mechanisms from animal studies. Front Hum Neurosci $\underline{7}, 687$

Reuter-lorenz PA, Jonides J, Smith EE, Hartley A, Miller A, Marshuetz C, Koeppe RA (1996): Age Differences in the Frontal Lateralization of Verbal and Spatial Working Memory Revealed by PET. J Cogn Neurosci $\underline{12}$, 174-187

Rodríguez C, González-Castro P, Cueli M, Areces D, González-Pienda JA (2016): Attention 
Deficit/Hyperactivity Disorder (ADHD) Diagnosis: An Activation-Executive Model. Front Psychol ㄱ, 1406

Rotzer S, Loenneker T, Kucian K, Martin E, Klaver P, von Aster M (2009): Dysfunctional neural network of spatial working memory contributes to developmental dyscalculia. Neuropsychologia 47 , 2859-2865

Roux F, Uhlhaas PJ (2014): Working memory and neural oscillations: Alpha-gamma versus theta-gamma codes for distinct WM information? Trends Cogn Sci 18, 16-25

Santarnecchi E, Muller T, Rossi S, Sarkar A, Polizzotto NR, Rossi A, Cohen R, Differences I, Capabilities I (2016): Individual Differences and Specificity of Prefrontal Gamma Frequency-tACS on Fluid Intelligence Capabilities. Cortex $\underline{75}$, 33-43

Sauseng P, Klimesch W, Schabus M, Doppelmayr M (2005): Fronto-parietal EEG coherence in theta and upper alpha reflect central executive functions of working memory. Int J Psychophysiol 57, 97-103

Sauseng P, Klimesch W, Freunberger R, Pecherstorfer T, Hanslmayr S, Doppelmayr M (2006): Relevance of EEG alpha and theta oscillations during task switching. Exp Brain Res $\underline{170}$, 295-301

Senior TJ, Huxter JR, Allen K, O’Neill J, Csicsvari J (2008): Gamma oscillatory firing reveals distinct populations of pyramidal cells in the CA1 region of the hippocampus. J Neurosci $\underline{28}, 2274-2286$

Shah A, Jhawar SS, Goel A (2011): Analysis of the anatomy of the Papez circuit and adjoining limbic system by fiber dissection techniques. J Clin Neurosci 19 2 , 289-298

Siegel M, Donner TH, Engel AK (2012): Spectral fingerprints of large-scale neuronal interactions. Nat Rev Neurosci $\underline{13}, 20-25$

Simons JS, Spiers HJ (2003): Prefrontal and medial temporal lobe interactions in long-term memory. Nat Rev Neurosci $\underline{4}, 637-648$

Skirrow C, Cross JH, Harrison S, Cormack F, Harkness W, Coleman R, Meierotto E, Gaiottino J, Vargha-Khadem F, Baldeweg T (2015): Temporal lobe surgery in childhood and neuroanatomical predictors of long-term declarative memory outcome. Brain $\underline{138}, 80-93$

Sohn M, Ursu S, Anderson JR, Stenger VA, Carter CS (2000): The role of prefrontal cortex and posterior parietal. PNAS $\underline{\text { 97, }}$ 13448-13453

Srinivasan R, Winter WR, Nunez PL (2006): Source analysis of EEG oscillations using highresolution EEG and MEG. Prog Brain Res $\underline{159}$, 29-42 
Sweeney-Reed CM, Zaehle T, Voges J, Schmitt FC, Buentjen L, Kopitzki K, Hinrichs H, Heinze HJ, Rugg MD, Knight RT, Richardson-Klavehn A (2015): Thalamic theta phase alignment predicts human memory formation and anterior thalamic cross-frequency coupling. Elife $\underline{4}, 1-9$

Szucs D, Devine A, Soltesz F, Nobes A, Gabriel F (2013): Developmental dyscalculia is related to visuo-spatial memory and inhibition impairment. Cortex $\underline{49}, 2674-2688$

Takeuchi H, Sekiguchi A, Taki Y, Yokoyama S, Yomogida Y, Komuro N, Yamanouchi T, Suzuki S, Kawashima R (2010): Training of Working Memory Impacts Structural Connectivity. J Neurosci $\underline{30}$, 3297-3303

Teyler TJ, DiScenna P (1987): Long-Term Potentiation. Annu Rev Neurosci 10, 131-161

Thut G, Schyns PG, Gross J (2011): Entrainment of perceptually relevant brain oscillations by non-invasive rhythmic stimulation of the human brain. Front Psychol 2,170

Thut G, Miniussi C, Gross J (2012): The functional importance of rhythmic activity in the brain. Curr Biol 22, R658-R663

Todd JJ, Marois R (2004): Capacity limit of visual short-term memory in human posterior parietal cortex. Nature $\underline{428}, 751-754$

Uhlhaas PJ, Singer W (2010): Abnormal neural oscillations and synchrony in schizophrenia. Nat Rev Neurosci $11,100-13$

Violante IR, Li LM, Carmichael DW, Lorenz R, Leech R, Hampshire A, Rothwell JC, Sharp DJ (2017): Externally induced frontoparietal synchronization modulates network dynamics and enhances working memory performance. Elife $\underline{6}$, pii: e22001

Vossen A, Gross J, Thut G (2015): Alpha power increase after transcranial alternating current stimulation at alpha frequency (a-tACS) reflects plastic changes rather than entrainment. Brain Stimul $\underline{8}, 499-508$

Vosskuhl J, Huster RJ, Herrmann CS (2015a): Increase in short-term memory capacity induced by down-regulating individual theta frequency via transcranial alternating current stimulation. Front Hum Neurosci $\underline{9}, 257$

Vosskuhl J, Strüber D, Herrmann CS (2015b): Transkranielle Wechselstromstimulation. Nervenarzt $\underline{86}, 1516-1522$

Vosskuhl J, Huster RJ, Herrmann CS (2016): BOLD signal effects of transcranial alternating current stimulation (tACS) in the alpha range: A concurrent tACS-fMRI study. Neuroimage 140, 118-125

Voytek B, Knight T (2015): Dynamic network communication as a unifying neural basis for cognition, development, aging, and disease. Biol Psychiatry 77, 1089 
Wang M, Gamo NJ, Yang Y, Jin LE, Wang X-J, Laubach M, Mazer JA, Lee D, Arnsten AFT (2011): Neuronal basis of age-related working memory decline. Nature $\underline{476}, 210-213$

Watson B, Buzsáki G (2013): Sleep, memory and brain rhythms. Daedalus 142, 26-47

Wayne R V., Hamilton C, Huyck JJ, Johnsrude IS (2016): Working memory training and speech in noise comprehension in older adults. Front Aging Neurosci $\underline{8}, 49$

Westerberg H, Klingberg T (2007): Changes in cortical activity after training of working memory - a single-subject analysis. Physiol Behav 22, 186-192

Westerberg H, Hirvikoski T, Forssberg H, Klingberg T (2004): Visuo-spatial working memory span: A sensitive measure of cognitive deficits in children with ADHD. Child Neuropsychol 10, 155-161

Woods AJ, Antal A, Bikson M, Boggio PS, Brunoni AR, Celnik P, Cohen LG, Fregni F, Herrmann CS, Kappenman ES, et al. (2016): A technical guide to tDCS, and related noninvasive brain stimulation tools. Clin Neurophysiol 127, 1031-1048

Zancada-Menendez C, Alvarez-Suarez P, Sampedro-Piquero P, Cuesta M, Begega A (2017): Requiring collaboration: Hippocampal-prefrontal networks needed in spatial working memory and ageing. A multivariate analysis approach. Neurobiol Learn Mem 140, 33-42 


\section{Acknowledgements}

I take this opportunity to express gratitude to my Supervisor Prof Dr Andrea Antal for sharing expertise and for the help and support. I am also grateful to my second committee member Dr Peter Dechent for a pleasant cooperation. My sincere thanks go to my Co-Supervisor Dr Ivan Alekseichuk for the continuous guidance and assistance. 


\section{Curriculum Vitae}

\section{Personal details}

My name is Stefanie Corinna Pabel, I was born on August 27 $7^{\text {th }}$ in 1991 in Tübingen, Germany, as the second child of my mother Dr. Astrid Ortrun Pabel and my father Dr. Dr. Helmut Pabel. My father is a pediatrician and works in his doctor's office in Herford, Germany, and my mother is a dentist and teaches in a vocational school in Bünde, Germany. My siblings' names are Annika Carolin Pabel and Marc André Pabel.

\section{Education}

After completing my school career, I studied Biology and Anglistics for one semester at the University of Münster, Germany, from 2010 until 2011. My professional life has evolved with an apprenticeship from the $1^{\text {st }}$ of August 2011 until the $25^{\text {th }}$ of January 2013 as a medical assistant in Herford, Germany. Afterwards, in 2013, I enrolled in medical school at the Georg-August-University of Göttingen, Germany. From the $20^{\text {th }}$ of November 2015 until present, I began my doctorate at the Clinical Department of Clinical Neurophysiology, University Medical Faculty in Göttingen, under the supervision of Prof. Dr. Andrea Antal.

\section{Professional Experience}

Before passing the medical preliminary examination, I completed practical nursing training for three months. From 08/05/2014 - 09/03/2014, I worked at the Klinikum Herford. At the Mathilden Hospital Herford, Germany, I completed two trainings (02/17/2014 - 03/18/2014 and 02/14/2011 - 03/16/2011). In the winter term of 2014/2015, I worked as a student assistant at the Centre for Anatomy of the University of Göttingen.

After the preliminary examination, I completed my clinical traineeship at the Raphaelsklinik Münster, in the Department for Gastroenterology (08/17/2015 - 09/15/2015). The second clinical traineeship took place in my father's doctor's office from 09/16/2015 until 10/16/2015. At the Center of Social Paediatrics of the University Medical Faculty in Göttingen, I worked from 07/18/2016 - 08/01/2016. From the 09/09/2016 - 09/23/2016, I completed the next clinical traineeship at the Department for General, Visceral, Thoracic and Proctological Surgery, of the "Klinikum Herford". Finally, I completed the last traineeship (02/01/2017 02/28/2017) at the surgical emergency room of the Clemenshospital in Münster. From October 2016 until June 2018 I worked as a research assistant at the Clinical Department of Clinical Neurophysiology, of the University Medical Faculty in Göttingen. 